\title{
Spot tests: past and present
}

\author{
María Teresa Doménech-Carbó ${ }^{1}\left[\right.$ ] $\cdot$ Antonio Doménech-Carbó ${ }^{2}$
}

Received: 15 June 2021 / Accepted: 11 October 2021 / Published online: 27 December 2021

(c) The Author(s), under exclusive licence to Springer Nature Switzerland AG 2021

\begin{abstract}
Microchemistry, i.e., the chemistry performed at the scale of a microgram or less, has its roots in the late eighteenth and early nineteenth centuries. In the first half of the twentieth century a wide range of spot tests have been developed. For didactic reasons, they are still part of the curriculum of chemistry students. However, they are even highly important for applied analyses in conservation of cultural heritage, food science, forensic science, clinical and pharmacological sciences, geochemistry, and environmental sciences. Modern pregnancy tests, virus tests, etc. are the most recent examples of sophisticated spot tests. The present ChemTexts contribution aims to provide an overview of the past and present of this analytical methodology.
\end{abstract}

\section{Graphic abstract}

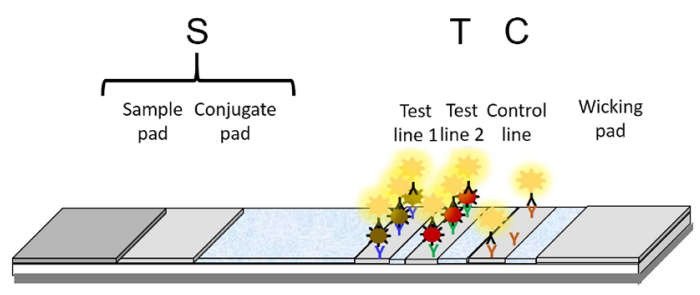

Keywords Spot tests $\cdot$ Microchemistry $\cdot$ Chemical microscopy $\cdot$ Dipstick immunoassay $\cdot$ ELISA $\cdot$ Microfluidics $\cdot \mu$ PAD . Lab-on-a-chip $\cdot$ Multiplexed tests $\cdot$ Smartphone spectroscopy

\begin{tabular}{|c|c|c|c|}
\hline \multicolumn{2}{|c|}{ Abbreviations } & CEC & Contaminants of emerging concern \\
\hline $\mathrm{ADH}$ & Alcohol dehydrogenase & CFA & Color filter array \\
\hline AFP & $\alpha$-Fetoprotein & CIS & Contact image sensor \\
\hline ALS & Alternative light source & CK-MB & Creatinine-kinase type MB \\
\hline AP & Acid phosphatase & CL & Chemiluminiscence \\
\hline \multirow[t]{2}{*}{ ASSURED } & $\begin{array}{l}\text { Affordable, sensitive, specific, user } \\
\text { friendly, rapid and robust equipment free }\end{array}$ & CMOS & $\begin{array}{l}\text { Complementary metal-oxide } \\
\text { semiconductor }\end{array}$ \\
\hline & and deliverable to end-users & CNP & Carbon nanoparticle \\
\hline BAC & Blood alcohol content & CNT & Carbon nanotube \\
\hline BNP & B-type natriuretic peptide & $\mathrm{COF}$ & Covalent organic frameworks \\
\hline CAP & Cystine aminopeptidase & $\mathrm{CP}$ & Cellphone \\
\hline $\mathrm{CD}$ & Compact disk & CRP & C-reactive protein \\
\hline CEA & Carcinoembryonic antigen & $\mathrm{C} 60$ & Fullerene \\
\hline \multirow{2}{*}{\multicolumn{2}{|c|}{$\begin{array}{l}\text { María Teresa Doménech-Carbó } \\
\text { tdomenec@crbc.upv.es }\end{array}$}} & $\begin{array}{l}\text { DCPIP } \\
\text { DIC }\end{array}$ & Dichlorophenol-indophenol \\
\hline & & DIN & German Institute for Standardization \\
\hline \multirow{2}{*}{\multicolumn{2}{|c|}{$\begin{array}{l}\text { Institut de Restauració del Patrimoni, Universitat Politècnica } \\
\text { de València, Camí de Vera 14, 46022 Valencia, Spain }\end{array}$}} & DNA & Deoxyribonucleic acid \\
\hline & & DUID & Driving under the influence of drugs \\
\hline \multirow{2}{*}{\multicolumn{2}{|c|}{$\begin{array}{l}\text { Departament de Química Analítica, Universitat de } \\
\text { València. Dr. Moliner, } 50 \text {, Burjassot, } 46100 \text { Valencia, Spain }\end{array}$}} & ECL & Electrochemiluminiscence \\
\hline & & EIA & Enzyme immunoassay \\
\hline
\end{tabular}




\begin{tabular}{|c|c|c|c|}
\hline ELISA & Enzyme-linked immunosorbent assay & MS & Mass spectrometry \\
\hline \multirow[t]{2}{*}{ ePAD } & Electrochemical paper-based analytical & $\mu \mathrm{TAS}$ & Micro-total analytical systems \\
\hline & device & MWCNT & Multiwalled carbon nanotubes \\
\hline $\mathrm{EtG}$ & Ethyl glucuronide & NIJ & US National Institute of Justice \\
\hline FOBT & Fecal occult blood testing & NIR & Near-infrared \\
\hline FTIR & Fourier transform infrared & NMR & Nuclear magnetic resonance \\
\hline GAS & Group A Streptococcus & NP & Nanoparticle \\
\hline $\mathrm{GC}$ & Gas chromatography & NPS & New psychoactive substance \\
\hline GDA & Glycylproline dipeptidyl aminopeptidase & NR & Nanorod \\
\hline GGT & Gamma-glutamyl transpeptidase & OIA & Optical immunoassays \\
\hline GLORIA & Optical-read rapid immunoassay & PAA & Poly(acrylic acid) \\
\hline GR & Graphene & PAT & Phadebas Amylase test \\
\hline GTP & $\gamma$-Glutamyltransferase & p-DMABA & para-Dimethylaminobenzaldehyde \\
\hline $\mathrm{HbA0}$ & Human hemoglobin A0 & P-ELISA & Paper-enzyme-linked immunosorbent \\
\hline $\mathrm{hCG}$ & Chorionic gonadotropin & & assay \\
\hline HE4 & Human epididymis protein 4 & PEth & Phosphatidylethanol \\
\hline HIV & Human immunodeficiency virus & PMMA & Poly(methylmethacrylate) \\
\hline HLA & Human leukocyte antigen & POC & Point-of-care \\
\hline \multirow[t]{2}{*}{ HPLC-MS } & High-performance liquid chromatogra- & POCT & Point-of-collection testing \\
\hline & phy-mass spectrometry & PRA & Procion red amylopectin \\
\hline HSB & Hue-saturation-brightness & PSA & Prostate specific antigen \\
\hline HSV & Hue-saturation-value & QB & Quantum nanobeads \\
\hline ICP & Inductively coupled plasma torch & QD & Quantum dot \\
\hline \multirow[t]{2}{*}{ ICP-ES } & Inductively coupled plasma torch-emis- & RGB & Red-green-blue \\
\hline & sion spectroscopy & RIA & Radioimmunoassay \\
\hline \multirow[t]{2}{*}{ ICP-MS } & Inductively coupled plasma torch-mass & RNA & Ribonucleic acid \\
\hline & spectrometry & SAP & Seminal acid phosphatase \\
\hline $\mathrm{IgG}$ & Immunoglobulin $\mathrm{G}$ & SARS-CoV-2 & Severe acute respiratory syndrome corona- \\
\hline $\operatorname{IgM}$ & Immunoglobulin $\mathrm{M}$ & & virus 2 of the genus Betacoronavirus \\
\hline $\mathrm{IL}$ & Ionic liquid & SEM-EDX & Scanning electron microscopy-X-ray \\
\hline \multirow[t]{2}{*}{ Interpol } & International Criminal Police & & microanalysis \\
\hline & Organization & SERS & Surface enhanced Raman spectroscopy \\
\hline IR & Infrared & SPE & Screen-printed electrode \\
\hline \multirow[t]{2}{*}{ IUPAC } & International Union of Pure and Applied & SPR & Surface plasmon resonance \\
\hline & Chemistry & SVSA & Seminal vessel-specific antigen \\
\hline LAP & Leucine aminopeptidase & SWCNT & Single-walled carbon nanotubes \\
\hline LED & Light-emitting diode & TCA & Thermal contrast amplification \\
\hline LFIA & Lateral flow immunoassay & TLD & Thermal lens detection device \\
\hline $\mathrm{LOC}$ & Lab-on-a-chip & TMB & Tetramethylbenzidine \\
\hline MDMA & Methylenedioxy- $N$-methamphetamine & $\mathrm{TNF} \alpha$ & Tumor necrosis factor- $\alpha$ \\
\hline MFD & Microfluidic device & TNT & Trinitroglycerine \\
\hline MIA & Multiplex immunoassay & TRFNP & Time-resolved fluorescence nanoparticle \\
\hline MMWCNT & Magnetized multiwalled carbon nanotbes & UCNP & Up-conversion nanoparticle \\
\hline MNP & Magnetic nanoparticle & US & United States \\
\hline MOF & Metal organic frameworks & USB & Universal serial bus \\
\hline \multirow[t]{2}{*}{$\mu \mathrm{PAD}$} & Paper-based analytical device, & USB-OTG & Universal serial bus on-the-go \\
\hline & lab-on-a-paper & UV & Ultraviolet \\
\hline MPNP & Mesoporous nanoparticle & VOC & Volatile organic compounds \\
\hline MPQ & Magnetic particle quantification reader & WHO & World Health Organization \\
\hline MPS & Mesoporous silica & XRD & X-ray diffraction \\
\hline
\end{tabular}


2D

3D

Two-dimensional

Three-dimensional

\section{Introduction}

The term "spot test" designates analytical procedures carried out at the scale of a microgram or less and are aimed at identifying a chemical species or a family of chemical compounds. In a more restricted meaning, spot tests are based on chemical reactions that result in the production of any distinctive (generally colored) product, either as a spot on white paper, or in a droplet on a spot plate [1]. Spot tests belong to microanalytical chemistry, which comprises staining tests, flame assays, thin-layer and paper chromatography, and many other analytical approaches.

Today, despite the sophistication of modern instrumental analytical techniques, spot tests are very frequently used in many areas, namely, quality control in food and pharmaceutical analysis, environmental and water analysis, metal and mineral analysis in industry and geosciences, conservation and restoration of cultural heritage, forensic science, and screening tests in clinical analysis [2,3]. In fact, a variety of commercial compact systems (kits) based on spot tests are produced either in the form of tablets or as plastic strips (dipsticks) to identify and/or (semi) quantify substances such as glucose, nitrites, bilirubin, etc.

Apart from their intrinsic scientific and practical value, spot tests are important because they are at the origin of the development of two important concepts in analytical chemistry: (i) The differentiation between specific and selective analytical reactions and reagents, accepted and recommended by IUPAC (International Union of Pure and Applied Chemistry) in 1937 [4]. (ii) The concepts of limit of identification and dilution limit (or its reciprocal, the concentration limit) introduced by Feigl [1, 5].

\section{History: microchemistry and chemical microscopy}

The term microchemistry has been introduced for chemical reactions and analyses performed with less than a microgram/ microliter of the involved substances. For analytical purposes, this wide definition may include almost all modern instrumental techniques, but generally its use is restricted to analytical procedures not using instruments, but only chemical reactions and their visual observation.

The history of this branch of the analytical chemistry was studied by Herbert K. Alber. Alber distinguished an early microchemical period prior to 1900 and stated that "François-Vincent Raspail (1794-1878), established himself as the founder of

\footnotetext{
1 "Chemical microscopy" is a misnomer, especially because at the end of the twentieth century scanning electrochemical microscopy had been developed, which is really a chemical microscopy. What is
}

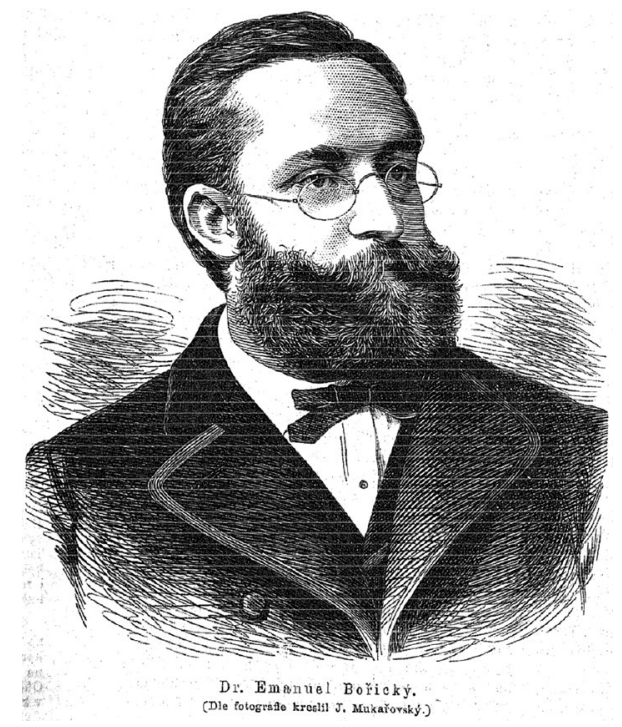

Fig. 1 Engraving of Emanuel Bořický (1878)

chemical microscopy, ${ }^{1}$ and he probably can be recognized as the first microchemist." According to Alber, the classical period of microanalysis ranges from 1900 to about 1945: "It is generally agreed that Friedrich Emich (1860-1940) at the Technical University in Graz, Austria, was the founder of classical microchemistry." [6].

It is difficult, however, to define the beginning of chemical spot tests. A remote reference can be found in Pliny the Elder (23/24-79 A.D.), who mentions an iron sulfate test in his Naturalis Historia [7]. Perhaps the first chemical color test can be attributed to Jean Jacques Colin (1784-1865, French chemist) and Henri-François Gaultier de Claubry (1792-1878, French chemist) who discovered in 1814 that iodine dies starch blue [7]. This test was applied by François-Vincent Raspail (1794-1878, French chemist and botanist) to sections of grasses, and he developed color tests to detect sugar, oil, albumin, resin, and protein to be combined with the microscopy examination of cell cultures [8]. In fact, with that he is the founder of both histochemistry and microchemistry [7].

Most of this line of research was developed throughout the nineteenth century under the perspective of the chemical microscopy, defined by the US chemist Emile Monnin Chamot (1868-1950) in 1915 as the application of the microscope to the solution of chemical problems [9]. This branch of microchemistry was developed with significant contributions of Theodore George Wormley (1826-1897, US chemist), Emanuel Bořický (1840-1881, Czech mineralogist and geologist) (Fig. 1), Theodor Heinrich Behrens (1842-1905,

Footnote 1 (continued)

called "chemical microscopy" in older (and even modern) literature is in fact optical microscopy applied in chemistry. 


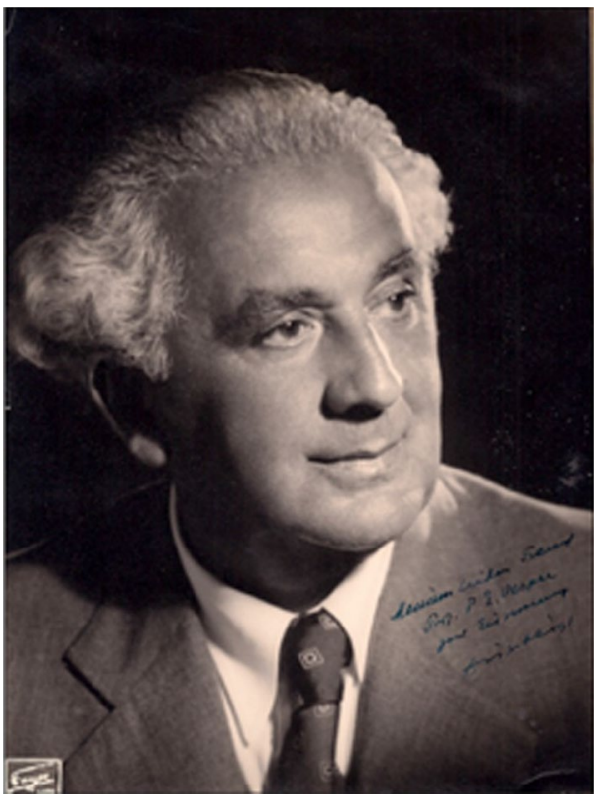

Fig. 2 Fritz Feigl

German chemist), Pieter Dirk Cornelis Kley (1871-1935, Dutch chemist), and Leopold Rosenthaler (1875-1962, German-Swiss pharmacist), among others [8].

Concerning spot tests sensu stricto, the earliest report was probably the detection of uric acid by Hugo (Ugo) Schiff (1834-1915, German-Italian chemist) in 1859 using a filter paper strip impregnated with silver carbonate $[1,10]$. An earlier test was proposed for the identification of free chlorine using potassium iodide-starch paper by Friedlieb Ferdinand Runge (1794-1867, German chemist) in 1834. Friedrich Emich (1860-1940, Austrian chemist) [11,12] and Fritz Pregl (1860-1930, Austrian chemist, Nobel Prize in 1923) [13] developed considerably this field in the period 1917-1923, introducing new methodologies for the characterization of inorganic species by combination with organic reagents. This gave rise to the technique of spot tests (Tüpfelanalyse in German, Analyse de tâche or Réaction à la touche in French, Ensayo a la gota in Spanish, and Analise de toque in Portuguese) which was considerably expanded by Fritz Feigl (1891-1971, Jewish Austrian-born chemist) (Fig. 2) [14-19].

Feigl introduced new reactions for spot tests, including the use of organic compounds as precipitation, color, fluorescent (or fluorescent-quenching), and masking agents. $\mathrm{He}$ employed induced and catalytic reactions, as well as reactions in the gas phase and in solid state at elevated temperatures through contact with suitable solids or dissolved reagents. The formation of colored lakes via adsorption of dyestuffs on metal oxides and the use of pyroreactions were other contributions.
It is pertinent to underline the relationships of spot tests with other analytical issues. Indeed, first spot test made uses of capillarity and adsorption phenomena on filter paper $[20],{ }^{2}$ thus underlying the relation with paper chromatography and thin-layer chromatography [21]. In turn, many specific reagents used to visualize spots on chromatograms were derived from Feigl's spot tests.

\section{Analytical properties of spot tests}

Spot tests are, in general, conceived as chemical reactions resulting from bringing together a drop of an unknown (analyte) solution and a reagent solution on a suitable support, yielding precipitates, gas, colored substances, etc. This operation can be carried out according to diverse experimental procedures. Therefore, the number of available spot tests is really considerable. Solely in Feigl's books published between 1939 and 1972 the number of spot tests (organic plus inorganic) increased from ca. 400 to about 1900 [1]. Thus, establishing a classification of spot tests is a tough task. In qualitative organic analysis and on the basis of the classification proposed by Feigl, several groups of spot tests can be distinguished according to their aim [18].

In general, spot tests can be characterized in terms of sensitivity, limiting proportions, specificity, and selectivity. Some of these properties are considered in more detail hereafter.

\section{Inorganic vs. organic qualitative spot tests}

In qualitative inorganic spot tests, the detection of a metallic or non-metallic element provides sufficient information for solving the problem. In contrast, in organic spot tests the goal is the identification of a particular compound or a characteristic group (functional group) of the compound. Thus, a classification of the organic spot tests can be made according to the type of target species recognized in the test:

(a) Identification of characteristic organic compound types

(b) Identification of individual organic compounds

(c) Identification of functional groups forming the organic molecules

\footnotetext{
2 One of the first works of Feigl was done in collaboration with the Austrian chemist Rose Stern (1891-1962) (Soukup RW, Rosner R (2019) Scientific contributions of the first female chemists at the University of Vienna mirrored in publications in Chemical Monthly 1902-1919. Monatshefte für Chemie-Chemical Monthly 150: 961974). This paper was the first comprehensive study on the application of filter paper as support for carrying out spot tests due to the capillary effect, which enables the separation of different analytes present in the same sample. Thus, Stern can be considered a pioneering woman contributing to the development of the spot tests.
} 
(d) Identification of metallic and non-metallic elements being part of the organic molecules

(e) Recognition of the reactivity of the organic compounds: ignition or combustion behavior, acidic or basic character, redox reactivity, behavior towards reactive or nonreactive solvents

In qualitative inorganic microanalysis most of the spot tests are based on ionic reactions between inorganic compounds in aqueous solution. In qualitative organic microanalysis most spot tests are carried out by dissolving the unknown material in organic solvents. Alternatively, the unknown compound may be melted at elevated temperature, vaporized, or incorporated in heterogeneous systems. This variety of reaction media often has disadvantages such as incompleteness of the reactions, side reactions, and, in general, a slowing down of the reaction rate. Very satisfactory spot tests in qualitative organic microanalysis are based on pyro-reactions and condensation reactions in the $120-250{ }^{\circ} \mathrm{C}$ range, resulting in the release of characteristic products through ignition and sintering of the organic unknown with organic or inorganic reagents.

Binary reactions can be applied for either of the inorganic and organic reactant compounds for analytical purposes. In the case of inorganic spot tests this principle is applied using an organic molecule with reactive chelating groups with affinity to specific metallic ions so as to form colored insoluble salts or soluble complexes. Conversely, organic compounds, which include in their molecule these functional groups, can be identified by reacting with solutions of the specific metal ions in a suitable concentration (i.e., dioximes, acyloinoximes, or 8-hydroxyquinolines).

Systematic qualitative schemes in the form of groups of precipitation and groups of dissolved species play an important role in inorganic qualitative analysis whereas they are less important in qualitative organic analysis.

\section{Sensitivity and limit of identification of spot tests}

Although spot tests are conceived as qualitative analytical methods, they involve intrinsic quantitative information. The term sensitivity has not been unambiguously defined for spot tests: sometimes, the smallest detectable amount of material is meant; sometimes, the highest dilution of the test solution, at which detection is possible, without referencing the amount of used material. One of the earliest characterization of spot tests was given by the expression $[2,22]$

$x[S] y$,

where $S$ is the used technique, i.e., drop of solvent, capillary tube, filter paper, etc. $x$ is the amount of material dissolved in $y$ expressed in micrograms $(\mu \mathrm{g})^{3}$ and $y$ is defined as the volume of the sample solution expressed in milliliters. The limit of identification (quantity to characterize the sensitivity) proposed by Feigl [2, 22] was widely accepted for representing $x$, i.e., the sensitivity of the test irrespective of the solubility or insolubility of the analyte. This parameter is related to the concentration limit or its reciprocal the dilution limit. These parameters express the concentration (dilution) sensitivity of the test. The relationship between these parameters is expressed by the equation

Dilution limit $=\frac{\text { Limit of identification } x}{\text { Volume of test solution } 10^{4}}$,

where $x$ is given in micrograms and the volume of the solution is given in milliliters. Usually, it is expressed as

Dilution limit $=1: \frac{\text { Volume of test solution } 10^{4}}{\text { Limit of identification } x}$.

Therefore, a spot test is highly sensitive when the limit of identification is small and the limit of dilution large. In the cases where the analyte is highly insoluble, the limit of identification is sufficient for characterizing the quantity sensitivity of the spot test.

The sensitivity of a method, numerically expressed by the limit of identification and the dilution limit, is not a unique characteristic of the particular reaction. The reaction conditions, such as presence of interferents or other supplementary processes, the reaction time, etc., can notably influence their values. The sensitivity of the spot test can be improved by introducing changes in the working technique such as use of micro drops, working in capillaries, use of magnification instruments and microscopes instead observation at naked eye, as described later.

According to Feigl [2], the sensitivity, expressed as identification limit of direct and indirect spot tests for organic compounds, is in the range $0.4-1.0 \mu \mathrm{g}$ of the unknown material per drop (ca. $0.05 \mathrm{~mL}$ ). Spot tests can allow for the detection of amounts of inorganic analyte as little as $1 \mathrm{ng}$ in a drop (ca. $50 \mu \mathrm{L}$ ) of solution, corresponding to a dilution between 1:5,000 and 1:500,000,000. ${ }^{4}$ Accompanying

\footnotetext{
${ }^{3}$ In older books on microanalysis the unit $\gamma$ (gamma), corresponding to $1 \mu \mathrm{g}$, is used.

${ }^{4}$ The German Institute for Standardization (DIN) issued the standard DIN 32630:1994-10 entitled "Characterisation of chemical analytical procedures according to sample mass and content range" which classified analytical procedures according to the amount of sample as follows: A macro procedure operates with sample amounts up to $100 \mathrm{mg}$, semimacro or semimicro procedure operates with amounts in the range $100-1 \mathrm{mg}$, a micro procedure uses amounts between $1 \mathrm{mg}-1 \mathrm{~g}$, and a submicro or ultramicro procedure uses amount smaller than $1 \mu \mathrm{g}$. This standard was published in 1994 and was cancelled on 04/01/2016. According to the DIN32630:1994-10 classification, detections with spot tests (in both solid state or dissolved in a drop) are submicro or ultramicro procedures.
} 


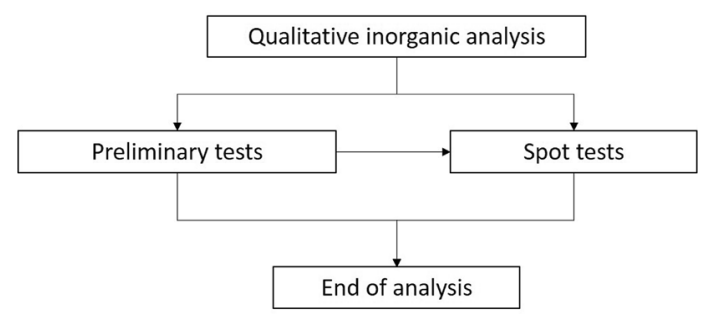

Fig. 3 Scheme of application of the different types of spot tests in qualitative inorganic analysis

materials in the sample can interfere with the test reaction and notably vary the sensitivity. For this reason, the limiting proportion has been defined as the ratio of the amount of analyte to the amount of accompanying material present in the sample. Therefore, the sensitivity, selectivity, specificity, and limiting proportion should not be attributed to a reaction or reagent but to a spot test.

\section{Methodology of spot tests}

\section{Bulk analysis}

The aim of a chemical analysis is to obtain information on the average composition of the entire object without taking into account its heterogeneities. This is the so-called bulk analysis. For this purpose, a sample representative of the object or material is selected. The sample is rendered uniform by subjecting it to an appropriate treatment, i.e., dissolution, powdering, etc.

Historically, the spot tests were designed for performing bulk analysis and this section is devoted to describe the fundamentals and experimental procedures developed for carrying out this type of analysis. In sections thereafter, a second type of microchemical tests will be presented that was specially developed for carrying out surface analysis.

\section{Qualitative inorganic analysis}

The methodology of qualitative analysis for inorganic materials varies depending on the nature of the target species: free elements, cations, anions, or insoluble compounds. According to Feigl [2], spot tests for qualitative inorganic analysis can be classified, irrespective of the nature of the analyte, as preliminary tests and direct spot tests. The former are fast trials that provide information on the presence or absence of certain types of compounds (oxidizing/reducing, $\mathrm{acid} / \mathrm{basic}$, etc.) and can consist of physical or wet chemical assays. Direct tests are chemical identification assays that can be performed solely, or after preliminary tests (Fig. 3).
This is a general classification of reactions used for the identification of inorganic analytes [2]:

(a) Precipitation reactions

(b) Complexing color reactions

(c) Reactions that yield fluorescent products or quench fluorescence

(d) Catalyzed and induced reactions

(e) Solid-state reactions at high temperature

(f) Reactions in the gas phase through contact with suitable solid or dissolved reagents

(g) Reactions based on interfacial effects (adsorption, capillarity, flotation)

A special type of spot tests based on a precipitation reaction is the so-called crystal or crystalline test. In this assay, the crystals of the product formed have a characteristic morphology that is inspected with the help of a microscope.

The identification of metallic ions by spot tests can be carried out applying a systematic scheme of separation if suitable techniques are used. Here, the pioneering work of Nicolaas Schoorl (1872-1942, Dutch chemist) should be mentioned: he developed microanalytical schemes for separation of metallic ions of different groups of soluble and insoluble materials [23-25]. Figure 4 shows the scheme of separation proposed for metal cations present in artworks using the ring oven technique (see further down) [26, 27].

Another example of a systematic scheme of separation of metallic ions using spot test is that proposed by the American Society for Testing and Materials for the characterization of the composition of alloys and metals [28]. The scheme proposed starts with a preliminary test of magnetic properties and follows with spot tests based on the color formed when the sample is subjected to a sequence of chemical attacks with pure acids at different degrees of dilution with water or specific reagents.

The sensitivity of tests for cations and anions is determined using standard solutions of high purity that are systematically diluted. For highly diluted samples a preconcentration is necessary to reach the range in which the analyte can be detected. For this, evaporation of the solvent is the most usual method. Another possibility is to use so-called collectors that are solids with the ability of fixing on their surface traces of the sample by means of precipitation or adsorption. Then, the spot test is conducted directly on the surface of the collector or, alternatively, the analyte can be put again into solution in a small volume of solvent. Sensitivity of the reactions of identification of ions can be decreased by the presence of masking agents in the sample that form complexes with the analyte. This setback can be avoided by introducing a pretreatment consisting of the addition of a demasking agent that forms a stable soluble or insoluble compound with the masking agent. Other interferences can 
Fig. 4 Separation scheme using the ring oven technique. Adapted from [27]

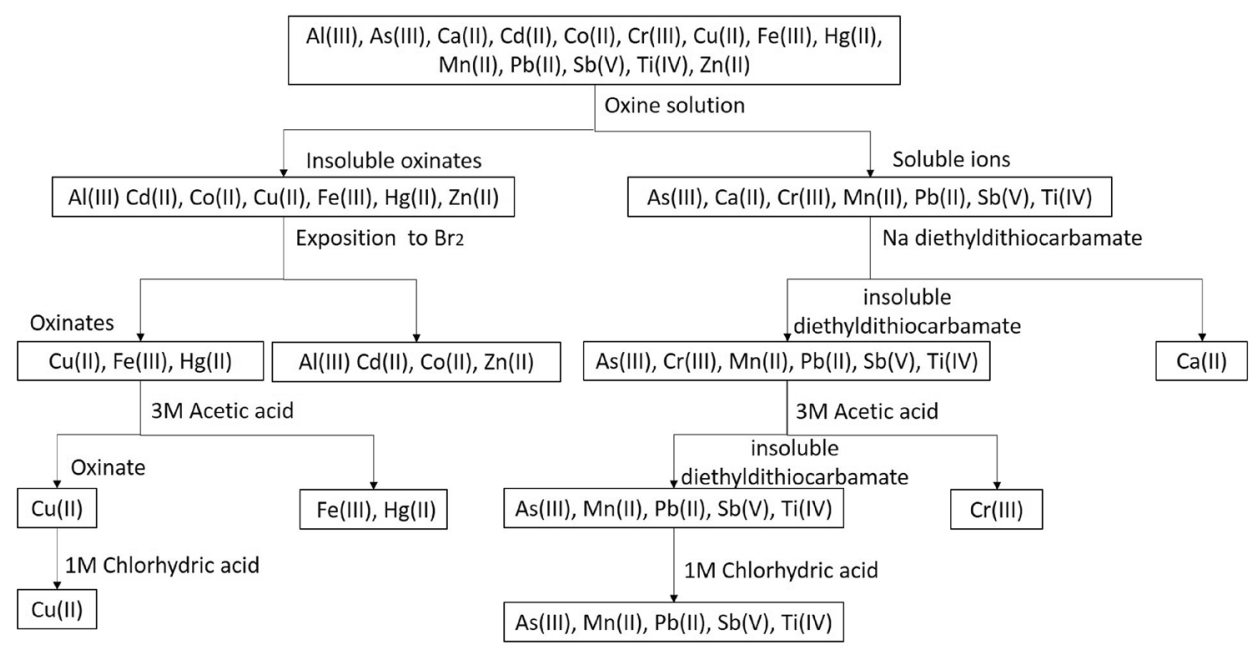

be prevented by adjusting the $\mathrm{pH}$ as many reactions are $\mathrm{pH}$ dependent.

\section{Qualitative organic analysis}

According to Feigl [18] spot tests for qualitative organic analysis can be classified into preliminary or exploratory test, direct and indirect spot test (Fig. 5).

Preliminary tests in organic analysis are, similarly to inorganic analysis, fast assays that require minimum amount of sample and provide valuable information about the organic matter in study for selecting the suitable spot test. For instance, a solubility test with water or organic solvents conducted on a paint sample from an altarpiece can indicate the hydrophilicity (proteinaceous) or hydrophobicity (lipid) of the binding medium. A specific follow-up spot test for proteins or dried oils should be used for rejecting or confirming the use of temper or oil technique by the artist.

In direct spot tests a functional group present in the target compound reacts with the reagent forming addition or condensation products, salts, oxidation or reduction products easily recognizable because of their characteristic color, fluorescence in ultraviolet or daylight, insolubility, etc. According to Feigl [18], the main types of reactions for direct tests are:

(a) Formation of an insoluble product or a colored or fluorescent soluble compound with an organic or inorganic reagent in aqueous or organic media

(b) Reaction with a solid or dissolved reagent in the gas phase

(c) Reaction of a solid with a solution

(d) Reaction of a solid with a gas

(e) Fusion reactions and reaction of a solid with a melt

(f) Sintering reactions and reaction of a solid with another solid (g) Color and fluorescence reactions related to interfacial effects (adsorption, capillary, flotation)

(h) Catalytic reactions in which the target compound acts as catalyst

(i) Catalytic reactions in which the reaction rate of the target compound is accelerated

In indirect spot tests, the reaction of the reagent with the functional group in the target compound results in an intermediate compound that, in turn, can be identified in a second step by the formation of a salt, condensation compound, etc. According to Feigl [18], the main types of reactions for indirect tests are:

(a) Degradation reactions (thermal, hydrolytic, oxidative, reductive) that result in the formation of cleavage products that are detected by a direct test

(b) Synthesis of compounds that are detectable with a direct test of $(\mathrm{a}, \mathrm{g}, \mathrm{h})$ type

(c) Masking of reactions of a direct test type leading to the detection of specific functional groups that have been blocked to react

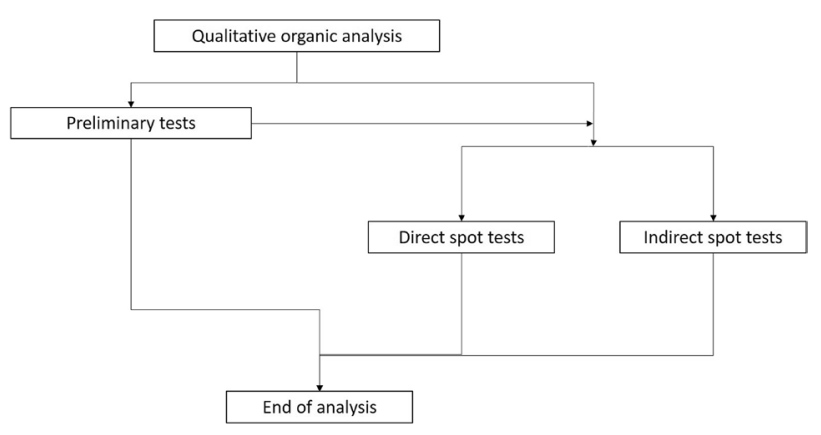

Fig. 5 Scheme of application of the different types of spot tests in qualitative organic analysis 
Both direct and indirect tests have advantages and disadvantages. Direct tests are faster than indirect ones and more sensitive as a single reaction takes place and there is scarce loss of sample during the analytical procedure; however, indirect tests have greater selectivity or specificity.

Reagents, reactions, and the corresponding procedures used on macro scale for analytical and preparative operations can be suitable for spot tests on the basis of their sensitivity, specificity, and selectivity. Sensitivity of the spot tests can be improved by modifying the macroscopic procedure of the preparation or synthesis reactions on which the test is based. Such modifications include changes in concentration and amounts of reagents, change in solvents, shortening of the reaction time, change of the reaction medium, e.g., from solution to gas phase, or increasing reaction temperature by conducting the spot test in melts or as a solid-state reaction.

Other factors affecting sensitivity of the detection of organic compounds or functional groups are occurrence, speed, and extent of the chemical reaction as well as discernability of the characteristic reaction product by its color, solubility, volatility, fluorescence, influence of the structure of the molecule (aliphatic or aromatic), etc.

\section{Preliminary tests}

Preliminary tests, also called exploratory or orientation tests, can be defined as simple tests that precede the chemical examination with direct or indirect spot tests carried out on both inorganic and organic materials. The main advantage of the preliminary tests is that they provide valuable information in case of both positive and negative results. These tests, as described hereafter, provide information on physical properties, solubility, acid-base or redox behavior, among other characteristics of the sample, that allow the analyst to select suitable spot tests for identifying the target compound, thus avoiding the implementation of a battery of tests that cover the wider range of possible results conceivable a priori. Combination of several preliminary tests can be helpful for providing useful clues so that the analyst can select the most suitable spot test for an unambiguous identification. Sometimes, preliminary tests can suffice for completing the analysis. This is the case when a confirmation of a certain element, compound, or class of compound is required. For instance, the presence of an element such as sulfur in an inorganic material or the identification of the type of organic compound used as adhesive in an old relining operation in a canvas painting (vide infra).

Inorganic analysis The preliminary tests proposed by Feigl [2] can indicate the presence or absence of certain types of compounds (oxidizing/reducing, acidic/basic, etc.). In the ignition tests, the finely powdered sample undergoes a characteristic change of color or sublimates, releasing vol- atile compounds easily identifiable with a second test. The sample, which is located in a spoon or crucible, is directly heated in the presence of air and the resulting changes are observed. In case of samples of rocks and minerals, the flame test indicates the presence of certain chemical element by distinct colors of the flame. In general, when inorganic substances are heated and they do not change color or disintegrate, the presence of mercury or volatile compounds such as ammonium salts, carbonates, etc., hydrates, or organic compounds can be excluded. If the sample melts, the presence of nitrites, nitrates, chlorates of alkali metals, among other salts, is most probable. If the sample totally or partially chars, i.e., forms carbon, the presence of organic matter, metal salts of organic acids, or organometallic compounds, among others, is indicated. If the sample changes its color without charring, presence of compounds of heavy metals must be suspected. When the sample is heated in an ignition tube several changes can be observed, such as condensation of water at the walls of the tube, formation of colorless or colored vapors, or appearance of a sublimate, which confirms the presence of salts containing water of crystallization, volatile acid, or ammonium salts and iodine, respectively, among other compounds.

Most of the preliminary tests are based on wet reactions. Soluble or insoluble basic materials can be identified by the increase of the $\mathrm{pH}$ of a solution of $\mathrm{Ni}$-dimethylglyoxime complex that induces the precipitation of the complex $\mathrm{Ni}$ dimethylglyoximate. Sometimes the preliminary test can involve pyrolytic processes as the test for sulfur-bearing compounds in which the sample is heated at $500{ }^{\circ} \mathrm{C}$ in the presence of calcium oxalate. The vapors of carbonyl sulfide can be recognized easily with a paper strip impregnated with lead acetate.

Organic analysis Often the material to be analyzed is a complex mixture of organic compounds. In such instances, a prior separation step must be carried out. There are a number of separation techniques available, such as distillation, sublimation, or adsorption. Most of them are basic mechanisms of chromatographic techniques. After the target compound is isolated, a specific identification reaction can be performed. Nevertheless, the separation operations are time consuming and they can involve an unaffordable loss of material. An alternative is preliminary tests that can precede the chemical examination with direct or indirect spot tests. They are particularly useful procedures in organic analysis owing to the wide number and variety of compounds for which there are not systematic schemes of analysis like in inorganic analysis.

For organic materials, the preliminary tests are classified into non-chemical tests, solubility tests, and chemical preliminary tests. Whereas non-chemical tests are based on the 
recognition of physical properties of the sample, chemical preliminary tests rely on chemical reactions.

Non-chemical tests The organoleptic examination of the sample is the simplest preliminary check, and, at the same time, this assay provides important information. Since organoleptic properties are those that affect the human senses taste, sight, smell, and touch, extreme care has to be taken to avoid intoxications. In fact, organoleptic examinations of chemicals are now strongly discouraged. They were common in the past, and so still during Feigl's lifetime. The observation of changes can be made by the naked eye or with the help of a magnifying glass or microscope (today there are easily available portable universal serial bus (USB) microscopes, with reasonable magnification, that are controlled from smartphones, thus acquiring in situ microphotographs that can be sent to the laboratory via the internet). Apart from the homogeneity or inhomogeneity of the sample, the color is the main physical property. The color is very useful for selecting suitable spot tests for unambiguous identification. A blue organic pigment is most probably an indigoid or phthalocyanine compound. Thus, specific spot tests can be used to discriminate the pigment type in a second step.

In forensic sciences, it is common to examine the crime scene with alternative light sources (ALS) that are devices providing monochromatic light at specific wavelengths for detecting materials such as biological fluids, fibers, or latent prints that exhibit luminescent properties. Spraying the crime scene with fluorescein before ALS illumination can be necessary for defining or enhancing patterns not visible but suspected to be present. These devices were pioneered by the Royal Canadian Mounted Police in the 1970s. Similarly, in conservation of cultural heritage it is common to examine the macroscopic objects under ultraviolet (UV) illumination to test the presence of fluorescing components. Observation of the fluorescence of microsamples can be performed using optical microscopes that are equipped with a UV lamp. The texture is another important physical property that can provide clues about the composition of the sample. Smoothness, roughness, and crystallinity of the sample are also important indicators. Odor is another important organoleptic property. The odor allows one to classify the volatile compounds present in the sample into ten different types that include a number of organic families of compounds (i.e., cacodylic odor is associated with cacodyl and trimethylamine compounds).

Another type of non-chemical test is based on the melting and sublimation properties of organic compounds. For instance, the identification of the type of adhesives used in old relining operations of ancient paintings on canvas can be satisfactorily carried out by determining the melting point. Before the twentieth century, waxy adhesives were commonly used in restoration. These adhesives consisted

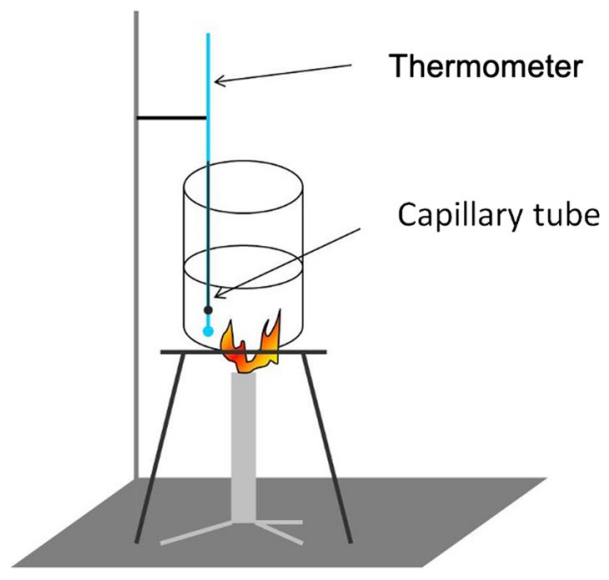

Fig. 6 Experimental setup for determining the melting point of a relining adhesive

of waxes alone or combined with other substances like terpenoid resins, etc. Waxy adhesives underwent stiffening and browning on ageing. Despite being applied on the back of the textile support, the adhesives often infiltrated throughout the multilayered structure of the painting, resulting in a notable change of the visual appearance of the artwork. For this reason, these adhesives fell into disuse and were progressively replaced by adhesives based on synthetic polymers when a painting needed reinforcement of its damaged canvas support. Identification of old waxy adhesives is easily conducted by determining their low melting points (below $100{ }^{\circ} \mathrm{C}$ ). Although nowadays a variety of instruments for determining the melting point are commercially available, a simple experimental setup, as that shown in Fig. 6, provides an accurate determination of the melting temperature. A few micrograms or less of the sample are put in a glass capillary with a sealed end. The capillary tube and a thermometer are placed in a water bath (capillary forces keep the capillary attached to the thermometer). Then, the water bath is slowly heated and the melting point of the sample is easily recognized when the sample changes its appearance from opaque to translucent. It is interesting to note that this test also indicates whether the sample is a single phase or a phase mixture. If the range in which the sample melts is not greater than $1 \mathrm{~K}$, the sample is probably a pure substance. If the sample does not melt uniformly or the melting range exceeds $1 \mathrm{~K}$, a mixture of compounds is present. The melting point can be confirmed during the subsequent cooling of the bath. Then the opposite change in the appearance of the sample is observed. Tables 1 and 2 summarize averaged values of melting temperatures of the main families of organic compounds used as adhesives in artworks and values for specific waxes, respectively [29]. Other groups of organic compounds have melting points above $100{ }^{\circ} \mathrm{C}$, for which 
Table 1 Average values of the melting points of the main groups of compounds used as adhesives and binding media of artwork in the past [29]

\begin{tabular}{lll}
\hline Temperature $\left({ }^{\circ} \mathrm{C}\right)$ & Family & Changes observed in the sample \\
\hline 60 & Waxes & Melting \\
120 & Terpenoid resins & Melting and darkening \\
$140-180$ & Oils & Melting and darkening \\
200 & Egg & Partial melting \\
200 & Plant gums, starch, dextrins, casein, and & Partial combustion resulting in \\
& animal glue & a solid residue \\
\hline
\end{tabular}

Table 2 Melting temperature of specific waxes [29]

\begin{tabular}{llllll}
\hline Type of wax source & Temperature $\left({ }^{\circ} \mathrm{C}\right)$ & Type of wax source & Temperature $\left({ }^{\circ} \mathrm{C}\right)$ & Type of wax source & Temperature $\left({ }^{\circ} \mathrm{C}\right)$ \\
\hline Mineral & & Vegetal & & Animal \\
Ceresin & $61-78$ & Candelillia & $65-69$ & Beeswax & $62-70$ \\
Montana wax & $76-92$ & Carnauba & $83-91$ & Chinese wax & $65-80$ \\
Ozokerite & $58-100$ & & & Spermaceti & $41-49$ \\
Paraffin & $30-60$ & & Lanolin & $38-42$ \\
\hline
\end{tabular}

other baths (glycerine, sand) or more sophisticated instrumentation is required for achieving higher temperatures. Identification of the specific type of wax needs sophisticated instruments that identify functional groups of the waxes. Nevertheless, the identification of the adhesive as a waxy material is often enough for selecting the necessary restoration treatment.

Burning and pyrolysis tests are other important types of preliminary tests for organic materials. These tests are conducted by placing the sample in an evaporating dish positioned over the flame of a Bunsen burner. Detonation or explosion is indicative, among others, of the presence of nitro compounds. The type of flame formed when the sample burns is indicative of the class of organic compound. For example, the formation of a smoky flame indicates the presence of aromatic compounds.

When the ignition takes place in the absence of air, pyrolytic processes such as removal of water, hydrolysis, cleavage, condensation, oxidation, or reduction reactions proceed. Formation of by-products due to reactions between products of the pyrolytic cleavage can also take place. Compounds such as hydrogen sulfide, acetaldehyde, volatile acids, or basic compounds, among others, are identified. The sample is placed in a reaction vial or tube covered with a lid or paper impregnated with the reagent; the vial is exposed to a flame underneath.

The identification of ignition residues also provides information on the composition of the studied material. Solubility of the residues is tested in water and acetic acid. For example, the salts of carboxylic acids, phenols, nitro compounds and oxime salts of bivalent metals, apart from alkaline earth metals, form carbonates and oxides

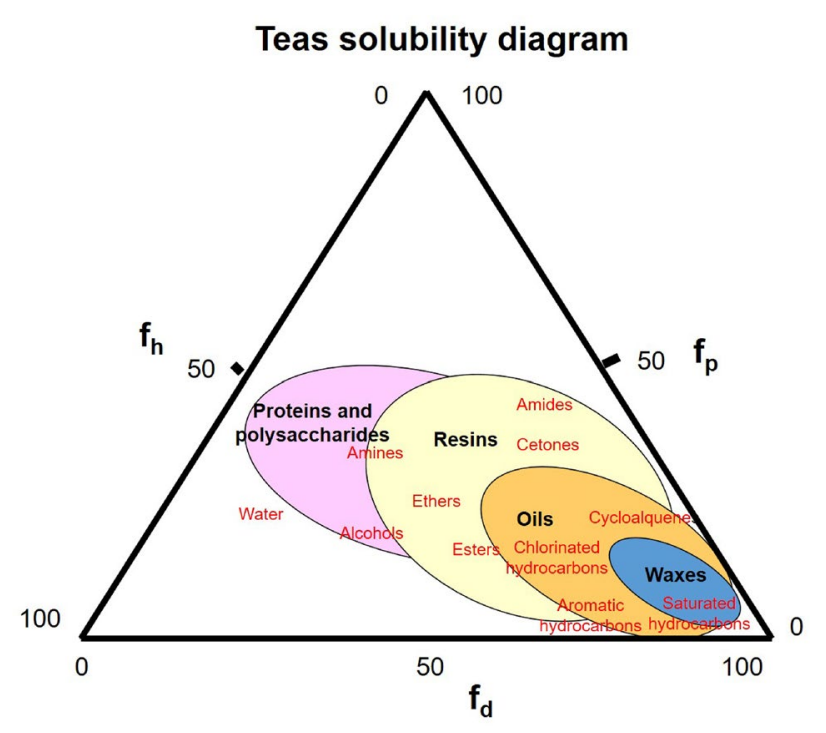

Fig. 7 Teas solubility diagram (1968). Partial solubility parameters: $f_{\mathrm{h}}$ hydrogen bonding forces, $f_{\mathrm{p}}$ polar forces and $f_{\mathrm{d}}$ dispersive forces

as residues, which are insoluble in water and soluble in diluted acetic acid.

Solubility tests These tests are of particular importance in the field of conservation of cultural heritage. Since the eighteenth century these tests, at that time called wiping tests, have been commonly used as preliminary tests, which are run prior to the cleaning treatment of a painting by conservators. Nowadays, these tests are rational solvent selections based on the theoretical model of partial solubility parameters [30-34] (Fig. 7). 
Chemical preliminary tests Although very similar in procedures and chemical fundamentals, distinction between chemical preliminary tests and direct spot tests can be established according to the greater selectivity of the latter. This applies, in particular, for chemical preliminary tests that are based on the same methodology and technique as the direct spot tests. As described previously, a physical property such as color, odor, melting point, or solubility/insolubility in specific solvents offers clues on what compounds are present in the sample in a non-chemical or solubility preliminary test. Similarly, the recognition of a specific element, acidic or alkaline behavior, or redox properties in a chemical preliminary test can indicate the presence of certain compounds or functional groups (i.e., if a preliminary test demonstrates the absence of sulfur in the sample, S-containing families of organic compounds such as mercaptans or thiols, $\mathrm{SC}$ or $-\mathrm{SO}_{3} \mathrm{H}$ groups are discarded). Conversely, identification of a metallic element reveals the presence of an organometallic compound.

\section{Quantitative analysis}

Quantitative determinations can be carried out successfully by means of colorimetry of colored complexes. Basically, the method consists of comparing the intensity of the color of the spot test performed on the sample and a series of standards, reference materials, or analyte solutions. The first spot colorimetric determination was conducted by the Russian chemist N. A. Tananaev ${ }^{5}$ (he wrote his name in Latin letters as Tananaeff) (1878-1959) in 1929 [2] by comparing the test carried out on the sample on a strip of paper or a spot plate with those on standard solutions. This procedure was improved further with the "confined spot test paper" method developed by Herman Yagoda (1908-1964) [35] and with the "extinction" method developed by Paul E. Wenger (1888-1962) [36]. More recently, Piechota and Drake Piechota [37] have developed an in situ semiquantitative spot test method for detecting chloride efflorescences that occur in artworks by measuring the degree of opacity of the silver chloride dispersion formed from a silver nitrate solution to which are added a few particles of efflorescences.

\footnotetext{
5 Nikolay Aleksandrovich Tananaev (Николай Александрович Тананаев) was the man who developed spot tests (in Russian called "капельный анализ", i.e., drop analysis) in the Soviet Union. He has published the following two books on this topic: (i) Тананаев НА (1948) Бесстружковый метод. Свердловск-Москва: Металлургиздат, 1948 (Tananaev NA (1948) Noncutting method. Sverdlovsk, Metallurgizdat: in this book Tananaev describes the analysis of metals and alloys without cutting them. A droplet of a reagent is applied to the surface and then the droplet is analyzed, e.g., by spot tests), (ii) Тананаев НА (1954) Капельный метод. Изд. 6-е перераб. и доп., М.-Л.: Госхимиздат (Tananaev NA (1954) Drop analysis, 6th ed, revised and extended, Moscow, Leningrad, Goskhimizdat).
}

A smartphone is used as light source. Degree of opacity is previously calibrated with standard chloride solutions. Nowadays commercial kits are available, which are based on spot tests in the form of tablets or dipsticks to identify and/or semiquantify a variety of substances and inorganic anions and cations. The dipstick has one or several pads, placed at the end of the stick, with the reagent absorbed (see hereafter). The test consists of dipping the pad into a drop of sample solution. The manufacturer provides a color scale for comparison of the experimentally observed color with calibrated color grades for semiquantification (Fig. 8). More recently, a series of methods that combine spot test with instrumental techniques have been successfully developed for various applications (see hereafter).

\section{Working techniques and materials}

General procedures for conducting the spot tests: Figure 9 depicts the general procedures for conducting the spot tests described here:

(a) A drop of the reagent solution and a drop of the test solution are brought together on a porcelain plate, well glass slide, or a porous support such as filter paper, silk, or cotton fiber, etc.

(b) A drop of the reagent solution is added to the spot of the reagent solution suspended in a porous support such as filter paper, silk, or cotton fiber.

(c) A drop of the reagent solution is added on the solid sample placed on a support.

(d) The reagent pre-impregnated in a dipstick is exposed to the vapors evolved from the solid sample or test solution placed on a support.

Pretreatments of the sample: Among the most common preliminary sample pretreatments are the following:

(a) Streak sampling is specific for solid samples and consists of rubbing the sample with a thin corundum stick so that small particles of the solid remain fixed on the end of the stick and the spot test can be conducted with this microsample.

(b) Comminution, mixing, grinding, and sifting are common methods for preparing solid samples. Steel-diamond or agate mortars and pestles are preferably used for this purpose but, if the sample is not too hard, porcelain or glass mortars can be used.

(c) Fusion of the powdered sample is carried out directly on a metal support or with the help of a fused bead formed on a metal loop, which comes into contact with the powdered sample. Then, the melt retained in the loop is brought to the flame or placed in a crucible that is heated in a furnace. 
Fig. 8 Commercial dipsticks for semiquantitative analysis of sulfate, chloride, carbonate, and nitrite/nitrate anions. The scale with the equivalence between anion concentration and developed color provided by the manufacturer is shown on the top of each frame and the dipstick with the result obtained from a real sample is shown below. The arrow correlates the experimental result obtained in the sample dipstick with the equivalent color-concentration. Note that sulfates and chloride dipsticks have more than one pad whereas nitrite and nitrate content can be measured with a single dipstick with two pads

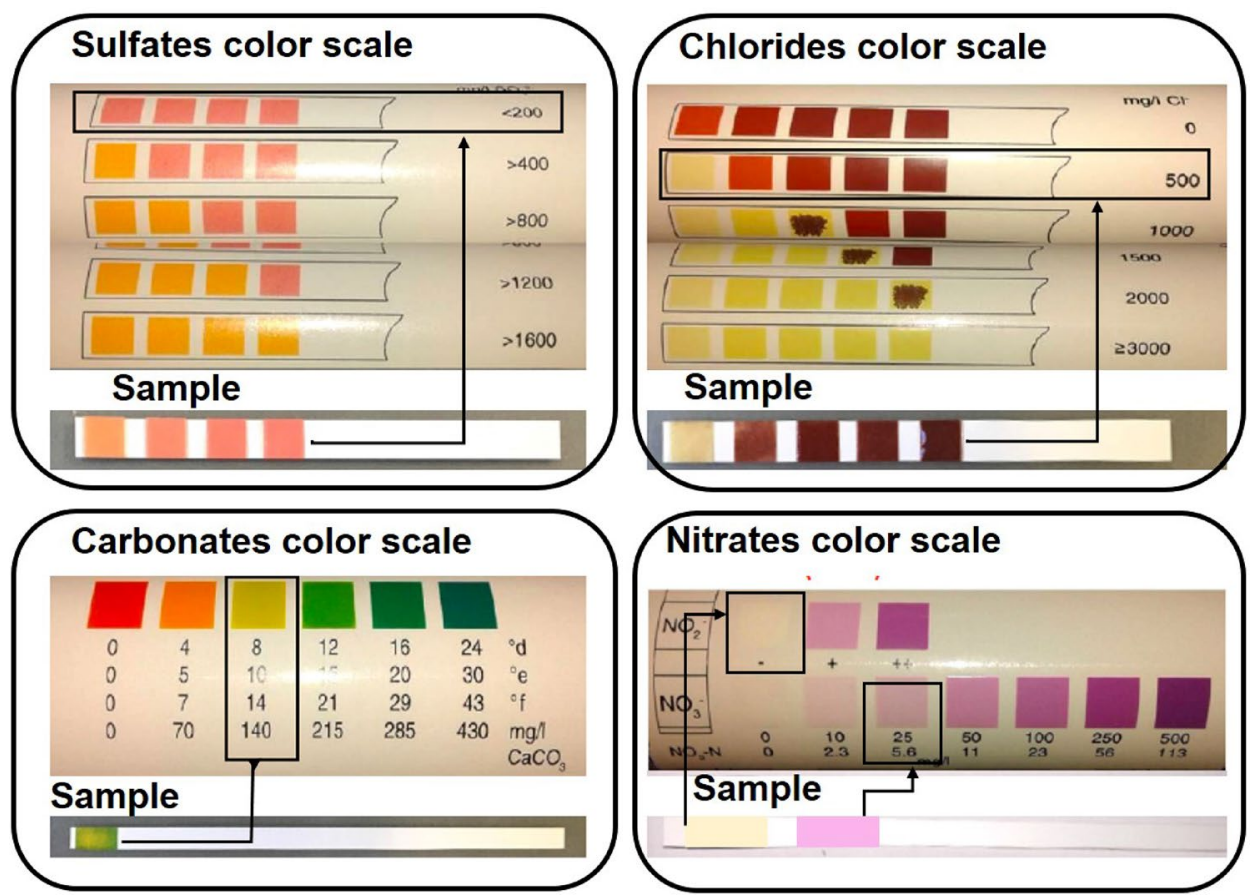

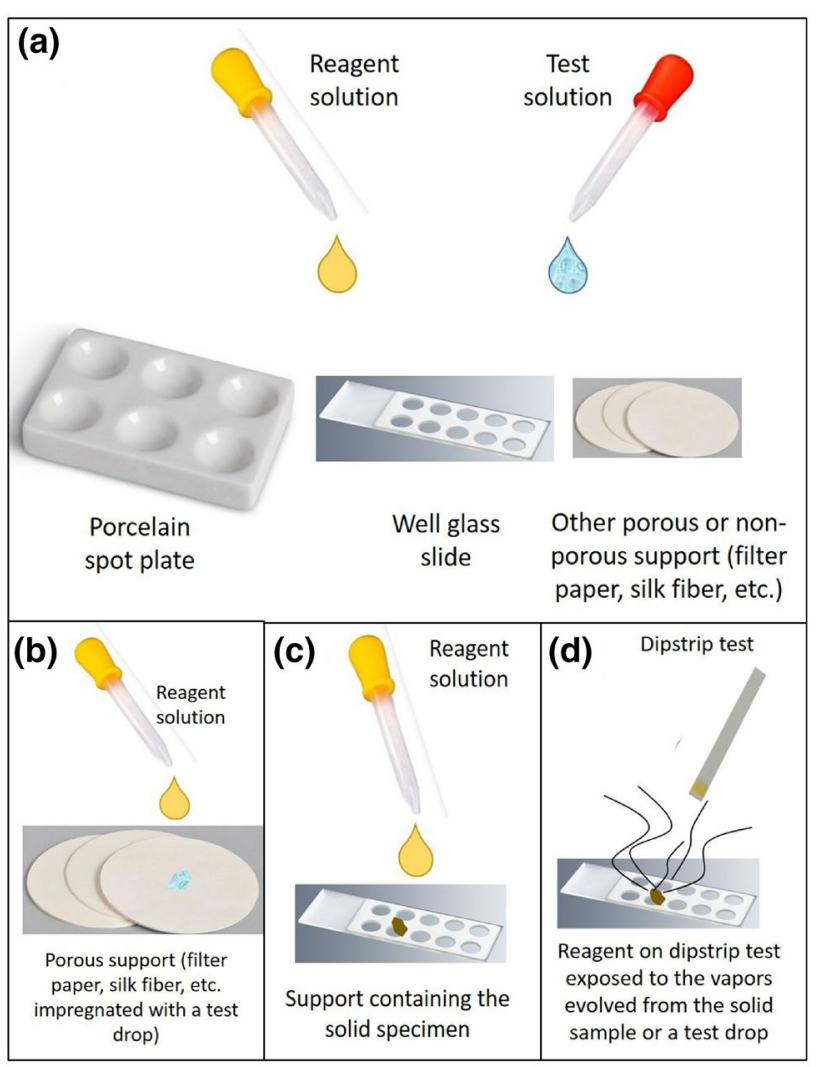

Fig. 9 Procedures for conducting the spot tests

(d) Drying of solids can be performed in ovens, heating blocks, or plates. A vacuum desiccator is used for a simple removal of moisture. (e) Evaporation of liquids for obtaining more concentrated solutions is performed directly on dishes or crucibles. More complex devices have been proposed in which the liquid is subjected to a pressure lower than that of the boiling point. Another common method for concentrating small amounts of solutions and liquid samples consists of directing a stream of air or an inert gas $\left(\mathrm{N}_{2}\right.$, Ar, He, etc.) to the surface of the liquid.

The most common separation pretreatments used in spot tests are:

(a) Capillary separation is used when two analytes, present in the sample, have different solubility in a liquid reagent medium. When the drop of the reagent is brought together with the spot of the sample suspended on a filter paper, the most insoluble analyte precipitates in

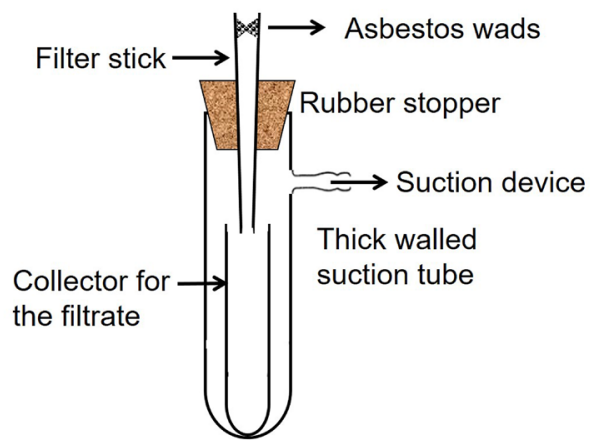

Fig. 10 Emich filter stick. Adapted from [2] 


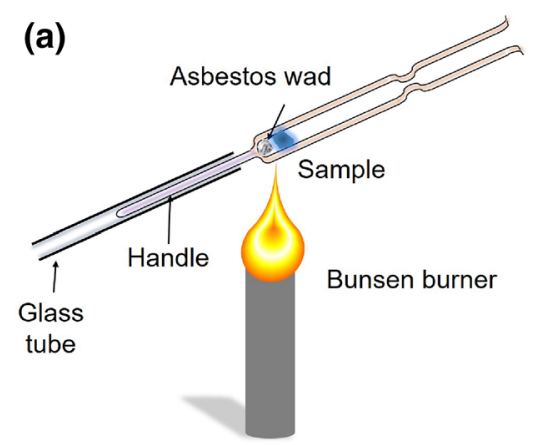

Fig. 11 Emich distillation tube is a piece of Pyrex glass constricted and sealed at the bottom with a length of ca. $60 \mathrm{~mm}$ and $4 \mathrm{~mm}$ of diameter. The sealed bottom is fixed to a glass stick that serves as handle inserted in a wider hollow glass tube for facilitating its manipulation. a The sample (1 to 3 drops) is placed with a pipet at

the center of the fleck whereas the other most soluble moves towards the periphery because of capillary forces and forms a ring around the initial spot of sample. Then, differentiating identification reactions are performed in the spot center and the ring.

(b) Sedimentation consists of a separation through precipitation methods. This procedure usually can be followed by another operation:

- Filtration is done in different ways depending on the characteristics of the analysis: (a) if the analyte is part of the solid residue or precipitate and, concomitantly, a large or small volume of liquid must be filtered, (b) if the analyte is part of the solution and, concomitantly, a large or small amount of solid must be filtered. If the analyte is part of a large volume of the liquid solution a simple assembly consisting of a pipet with a cotton plug in the end is used. The cotton retains the solid and the clear liquid passes through the pipet. Several more sophisticated instruments have been designed for filtering. A pipet with a special design containing a filter paper can be used in two ways for recovering the solid and the solution if both or one of them are of analytical interest. The Emich filter stick has been developed for recovering the solid filtered analyte (Fig. 10) [2].

- Centrifugation has some advantages for separation. In particular, the solid is liberated of most of the liquid or solution and the structure of the solid is not significantly altered in the separation operation. Microcentrifuge tubes with conical bottom are preferably used.

- Flotation is a method applied for the enrichment of a precipitate suspended in a large amount of solvent. This procedure is accomplished by shaking the dispersion with another solvent not miscible with the (b)

the sealed bottom onto a wad of asbestos previously positioned there. When the Emich tube, held with the handle, is heated with a Bunsen burner a ring of condensate is formed on the asbestos wad, which rises slowly. b After the ring of condensate passes the constriction it is collected with a pipet

former. The precipitate is concentrated at the interphase between both immiscible solvents.

(c) Liquid-liquid extraction of inorganic salts is performed with organic solvents in funnels or pipets. Similarly, other inorganic compounds that form chelate complexes can be extracted using organic solvents. Extraction of a liquid or solid melt with immiscible organic solvents enables separation of organic compounds. Liquid-solid extractions are performed in a Soxhlet extractor, extraction funnels, or pipets.

(d) Distillations can be performed using Emich distillation tubes $^{6}[38]$ (Fig. 11) or a simple distilling apparatus for small volumes of material that is made by connecting a crucible and a test tube placed in a support with a narrow angled glass tube.

(e) Chromatography is a separation method based on the different ability of the components of the sample to interact with a stationary phase (paper, thin-layer, particles packed in a column). The main advantage of this method is that different components of the sample are simultaneously separated. Further individualized spot tests enable the identification of each analyte.

(f) Chemical masking is based on the selective or specific ability of a complexing agent to form a soluble colored complex with the analyte that, in this way, is separated and identified.

(g) Electrochemical separation (electrophoresis) is based on the different behavior of analytes when, located in

${ }^{6}$ Gettler and Fine (Gettler AO, Fine J (1939) A Distillation Capillary. Ind Eng Chem 11: 469-470) and Morton and Mahoney (Morton A, Mahoney J (1941) Analysis of Single Drop of Liquid by Microfractionation Ind. Eng. Chem. 13: 494-498) developed more sophisticated adaptations of the Emich tube. 
a paper support, are subjected to an electric field that induces their migration.

Supports. In the early times spot test were carried out in a drop of a test solution previously prepared by dissolving the sample in a suitable solvent. Drops of the test and reagent solutions are brought together on a spot plate or a glass slide with well. This working technique was soon extended to other types of procedures based on solid materials (powders, smooth and porous surfaces, massive solids, etc.) or experimental setups (capillary tubes, fibers, etc.).

(a) Porous supports: the most commonly used support is a filter paper or another absorbent paper. This support provides a uniform medium for spreading and adsorbing the reagents throughout its capillary fibrous structure. The white background of the paper also improves the discernability of the changes due to the reaction (change of color, formation of a precipitate, etc.). Most spot tests based on a change of color are sensitive when conducted using a paper support but can be insensitive when carried out in test tubes or spot plates. The increase of the active surface of insoluble reagents finely dispersed on the paper is responsible for the enhancement of the sensitivity of the spot test. In some cases, such as the identification of permanganate in solutions of alkali chromate, the paper can also act as reagent. Nevertheless, the formation of a fleck is the result of the complex interplay of effects such as capillary spreading, diffusion, adsorption, swelling, etc. Therefore, the selection of a suitable paper is important because the sensitivity of the spot test can notably vary. Spot tests that require heating, fusion, sintering, evaporation, or reactions in organic solvents or in highly alkaline or acidic media are excluded from this type of support as the paper is attacked by these media.

The experimental procedure consists of pouring a drop of the liquid sample on the paper support so that the drop spreads as a result of surface forces until they are balanced. Then, a drop of the reagent is applied on the wet area so that the reaction proceeds and produces a colored product. A second method consists of the impregnation of the paper strip with the soluble or insoluble reagent. Then, the strip is completely dried. After this, the reagent strip is dipped in the test drop. $\mathrm{V}$-shaped strip tests have been proposed for this purpose. Alternatively, drops of the test and reagent solutions are brought together side by side so that the reaction proceeds in the line formed at the boundaries of both drops. Flecks or rings are observed within the spot formed by the reaction drops, where the reaction products locally accumulate as a result of the spot reaction, which enhance the discernability of the analytes. Three types of analyte identification by capillary spreading can be established: (a) identification by precipitation of the analyte; (b) separation based on differences of solubility between the analyte and other ions. Here, the analyte with the lowest solubility product precipitates in the center of the spot whereas other ions present in the sample, which have higher solubility product, precipitate in concentric peripheral zones; (c) separations based on differences in the colloidal behavior.

Notable improvement in sensitivity and reproducibility was achieved by Skalos [39] and Clarke and Hermance [40] using paper as support. The Akro method developed by Georgius P. Skalos (Greak chemist, 1915-?) uses a strip of paper with a triangular end (of ca. $5 \mathrm{~mm}^{2}$ ) where the spot test is conducted. The name "Akro method", introduced in German language publications, derives from the Greek word

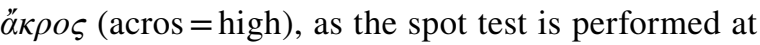
the pointed end of the paper strip, where the reagent and test solutions concentrate because of the very small paper volume. This method notably increases the limit of identification. If the sample drop is not sufficiently concentrated, the analyte is concentrated by evaporating the solvent of the sample drop (at ca. $50 \mu \mathrm{L}$ ) previously in a glass vial, by pouring a small aliquot of the sample drop into the tip of the Akro strip, and then heating it until the solvent is evaporated. Clarke and Hermance [41] introduced the use of close-textured papers impregnated with low solubility reagents. The sample is applied on the paper tests with a capillary burette. The same authors proposed a second method based on an apparatus that consists of a capillary burette connected to a flange and clamping assembly for holding the paper support impregnated with the sample on which a stream of the solution reagent is poured. This arrangement is suitable in the cases in which the analyte is highly diluted in the sample and, therefore, a large amount of reagent is needed to achieve suitable sensitivity. Another method developed by Yagoda [35] consists of applying a ring of paraffin on the paper strip and pouring the sample solution into the area framed by the ring so that the analyte remains concentrated in the delimited small area of the support as the hydrophobic paraffin avoids the capillary spreading of the solution.

Chromatographic spot tests based on the use of a strip of filter paper impregnated with basic or acidic alumina have also been proposed. The cations and anions ascend through the strip at different rates and thus they can be identified separately [42].

Currently, test strips also called dipsticks are abundantly used in various application fields of science and industry. The basic format of dipsticks includes 


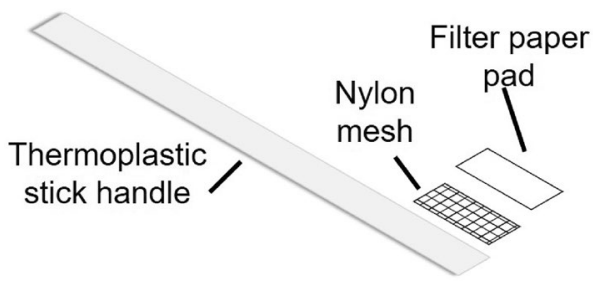

Fig. 12 Scheme of a dipstick that shows the different pad layers

a "detection zone" that consist of a pad made of filter paper impregnated with the reagents attached to the end of a carrier stick that acts as handle and is made of thermoplastic polymer (see carbonate dipstick in Fig. 8). The pad is fixed to the stick by a nylon mesh that prevents bleeding and ensures the uniform distribution of the sample and further color development (see Fig. 12). For quantitative purposes some dipsticks contain more than one pad with varying content and/or combination of reagents that enable quantitation according to the color changes in the pads (see sulfate and chloride dipstick in Fig. 8). Dipsticks with two or more pads for testing more than one analyte are commercially available (see nitrite/nitrate dipstick in Fig. 8).

Multilayered dipsticks are the most complex paperbased devices that enable one to illustrate how the customization with both stacking paper layers and lamination with a plastic film can improve the dipstick method. A top spreading layer comprising a porous polymer such as cellulose acetate is on top of the reacting zone and ensures the correct uptake and uniform distribution of the sample (Fig. 13). A pigment with high reflection power such as titanium dioxide is homogeneously distributed in this layer. In addition, adequate selection of the pore size prevents larger particles than those of the analyte from entering the layers beneath. The reagents are separately embedded in several intermediate hydrophilic layers of gelatin or agarose to avoid mutual influences among them. The successive reactions that take place between the analyte and the different reagents result in a colored complex formed in the lower reagent layer. At the bottom is located the stick made of a transparent polyester so that the measurement of the test color can be performed visually or with the help of an optical instrument. A relevant contribution by Hillscher et al., dedicated to functional materials for diagnostics, can be found in ChemTexts [43].

(b) Microtest tubes: Spot test based on sintering and fusion reactions, which can not proceed on paper supports, are conducted on this support. These spot tests have the advantage of increasing reactivity of solids at high temperatures. The sensitivity of the spot test
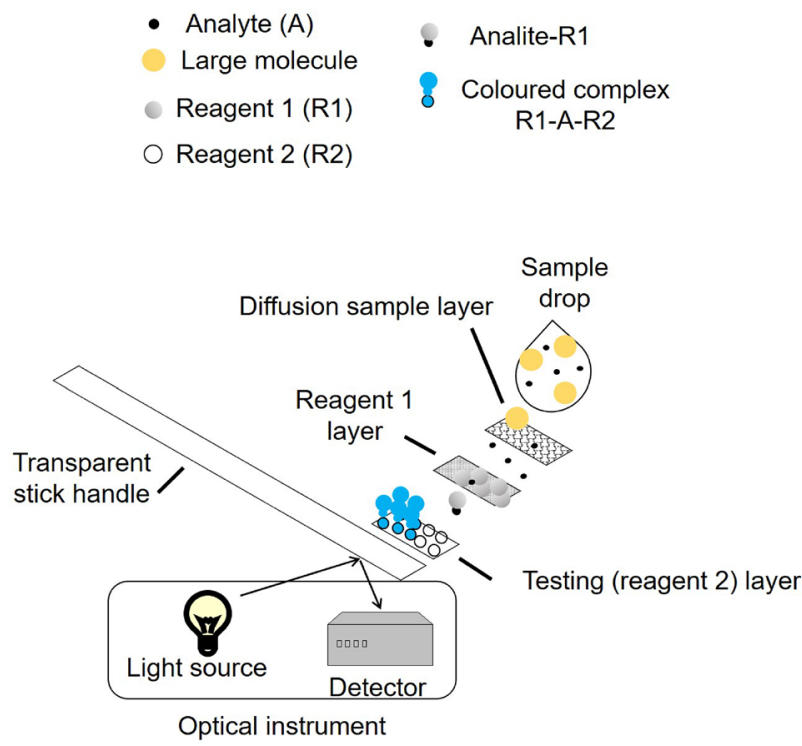

Fig. 13 Multilayered dipstick. The detection of a hypothetical analyte starts in the upper porous layer 1 that excludes the passage of molecules larger than the analyte. Colorless analyte (A) reacts with colorless reagent 1 (R1) in the layer underneath to form a colorless complex (A-R1). In the next layer the A-R1 complex reacts with the colorless reagent 2 (R2) and forms a blue R2-A-R1 sandwich complex. The transparent stick at the bottom enables visual detection of the color change or a more accurate measurement of the reflected light emitted by an optical instrument

conducted with aqueous test and reagent solutions can be improved by adding an organic solvent not miscible with water in which a colored analyte-reagent complex is extracted. A microtest tube is required in such instances.

(c) Metal equipment: Some spot tests require heating the sample at high temperature or a reactive system. Use of dishes, foils, crucibles, boats, spoons, loops, or wires made with metals such as nickel, nichrome, aluminum, steel, and preferably platinum is necessary in such instances.

(d) Other supports: Silk threads, gelatin foils, and paraffined porcelain dishes have been suggested as supports for spot tests.

(e) Other equipment and instrumentation: Apart from the support, a variety of laboratory material can be necessary for conducting a spot test: beakers, flasks (Erlenmeyer, for measuring, for suction, etc.), dishes (for crystallizing, evaporating, etc.), Barnes dropping bottles (a bottle fitted with a pipet), pipets and pipettes with removable tips, burettes, porcelain crucibles, vials, tubes (for macro and micro test, centrifugation, etc.), cover glasses, micro spatulas, needles, tweezers, micro sieves, micro distillation equipments, etc. 
Laboratory instruments such as balances, UV or infrared (IR) lamps, $\mathrm{pH}$ meters, ovens and furnaces, heating plates, Bunsen burners, heating blocks, water baths, centrifuges, ultrasonic baths, Vortex mixers, cooling blocks, microscopes, electrographic and melting point apparatus, etc. are also used.

\section{Special methods}

Ichnography, ring oven, Conway cell, and electro-spot testing are special methods proposed to improve the sensitivity and efficiency of the spot test.

(a) Ichnography: This method consists of applying a line (streak) of a few centimeters length with the solid material of the sample on the rough surface of a corundum or hard porcelain plate (like drawing a line on a touchstone for a gold assay). The material deposited on the plate is dissolved and then transferred to a filter paper impregnated with the reagent. A transparent sticky foil is used as transfer medium between the plate and the paper strip with the reagent. The term "ichnography" was coined by G. Skalos, referring to the Greek term for footprint, trace: i $\chi \nu o \varsigma$ (ichnos) [39, 44].

(b) Ring oven: This technique developed by Herbert Weisz (1922-2018) [26] is a modification of the conventional technique for conducting spot tests on paper. The main advantage of this technique is that the concentration and separation of a complex sample that includes a great number of ions (as many as 35 ) is allowed in a single drop of a few microliters of the dissolved sample with increased sensitivity. Initially, some of the ions precipitate in the center of the spot. Then, the unprecipitated ions are separated by washing them with suitable solvents from the center of the fleck to its periphery. Thus, the more soluble ions diffuse concentrically. To avoid the risk of excessive dilution of ions shifted along a wide area, the ring oven technique uses a thin aluminum ring, with diameter slightly larger than the original spot, which is placed on a base ring. The base ring is heated to evaporate the washing solvent, so that the ions are concentrated on the paper in a peripheral annular area (Fig. 14). The identification tests can be conducted in different sectors of the ring after cooling. The original spot of precipitated ions is cut out and placed on a new filter paper where the remaining ions are dissolved with suitable reagents followed by washing out into a new formed ring that is properly separated and tested. Figure 4 shows a scheme of separation using this technique.

(c) Microdiffusion Conway cell: On the basis of prior devices developed by Schlösing, Redrawn, and Fran-

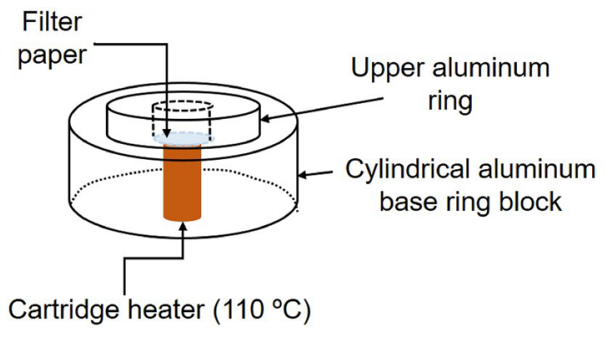

Fig. 14 Scheme of the ring oven

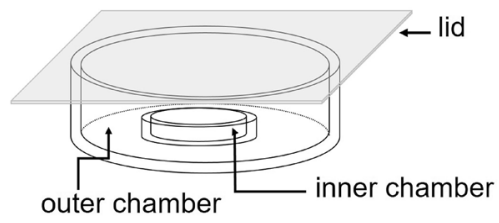

Fig. 15 Scheme of a simple microdiffusion Conway cell with two chambers

kel, Edward Joseph Conway (1894-1968) and Byrne [45] developed a microdiffusion cell for the microdetermination of volatile substances that was improved in later years. The apparatus consisted of a circular glass plate with one or two outer concentric chambers separated from the inner circular chamber by lower walls. The plate is sealed with a flat lid that rests on the outer wall allowing the diffusion of gas between the internal chambers. The gaseous product resulting from the reaction between the sample and a suitable reagent in the outer chamber exerts a certain tension on an absorbent placed in the inner chamber and thus is diffused towards there, where tension approaches zero. The identification reaction proceeds in the inner chamber with a suitable reagent (Fig. 15). This technique has been widely used for studying enzymatic activities and for the analysis of varied substances such as carbon monoxide, carbon dioxide, halogens, ammonia, alcohol, acetone, amides, amines, adenosine and their derivatives, lactic acid, or glucose [46].

(d) Electro-spot testing: This method is a special type of electrochemical separation also called electrolytic transfer or electro-transfer, which is based on driving ions from the phases of an ore or metallic sample that is conducting and ionizable into a paper reaction medium impregnated with a noninterfering electrolyte. Two microchemical techniques were developed in the first half of the twentieth century based on the electrotransfer phenomenon: the electro-spot testing and the electrographic testing. The former is described here. 


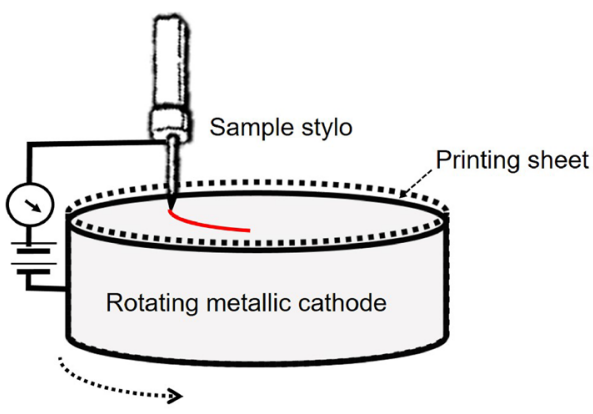

Fig. 16 Scheme of the experimental arrangement of the electro-spot tests by Fritz

Helmut Fritz [47] in 1929 developed a spot test method based on electro-transfer called the electro-spot test method (Elektro-Tüpfel-Methode in German). The first experimental arrangement developed is shown in Fig. 16. A paper impregnated with an electrolyte, and with a color-producing reagent if necessary, is placed on a rotatory cylindrical metal cathode. The sample is in the form of a stylus that contacts the paper sheet so that, when a potential is applied, oxidation of the atoms of the specimen results in a transfer of ions to the paper where they react and form a uniform streak on the moving paper. Measuring the current intensity in the circuit and the speed of the drum permits calculation of the amount of metal dissolved by applying Faraday's law. This method has the advantage of avoiding polarization as the electrolyte is continuously renewed.

For the identification of a single component in a metal or alloy removal of interferences by immersion of the spot print in solvents or in a solution containing masking reagents followed of washing and drying is required often. These steps are facilitated using printing media with a minimum wet strength to enable handling and with relatively open capillary structure such as Schleicher and Schüll 576 or Whatman 50 papers. A thicker backing paper such as Schüll 601 can be placed underneath the priming paper to increase its capacity for absorbing electrolyte, and to minimize possible interferences due to the contact with the metal electrode.

\section{Surface analysis}

The surface of a material is understood here as the outer layer of atoms or molecules of a solid material adjacent to a second phase, such as vacuum, air, or a liquid. The chemical composition varies through this layer, which has a thickness of 5-20 nm, until reaching the values characteristic of bulk material. Of course, compositional changes (a profile) can stretch up to a few micrometers into the material. The distribution of compounds in the surface of a material is not uniform and, commonly, each material is characterized by two-dimensional (2D) distributive patterns along the surface.
Examination and analysis of the surface of a material are of great interest because most of the chemical processes or physical properties such as corrosion, catalysis, or adhesion take place at the surface of the material. In other cases, thin layers of protective coatings of organic and inorganic nature are applied to the surface of the material. The object can also been subjected to mechanical processing such as grinding, polishing, or rolling that results in a change of the structure of the surface. For these reasons, surface analysis, which interrogates both the composition and the distribution of materials present on the surface and subsurface of a material, soon awaked the interest of scientists. Some of the oldest methods for studying the surface of an object are based on obtaining its surface analytical pattern. The analytical pattern may be defined as an imprint of the surface, interpreted in terms of its chemical composition, which is made on an external medium as a result of the controlled mechanism of its formation. The analytical patterns can be based on physical phenomena such as autoradiography, autoluminography, and magnetography, on chemical reactions, or on electrochemical processes. The last two types belong the chemical contact printing test, firstly developed and based on chemical reactions, and the electrographic testing based on electrochemical phenomena.

In the last decades, several highly sensitive surface analytical techniques have been developed (i.e., secondary ion mass spectrometry or scanning electrochemical microscopy), which afford 2D images and compositional mapping and surface depth profiles. These methods have displaced older surface analysis techniques based on chemical and electro-transfer printing. Nevertheless, it is of great interest to describe these pioneering techniques that were the starting point for the development of more advanced techniques of surface analysis.

\section{Chemical contact printing}

Baumann [48] published a chemical contact printing or imprint method in 1906. In the chemical contact printing technique the microstructural composition of the object surface is revealed by applying a suitable chemical etching reagent which is impregnated in a printing sheet (Fig. 17a). The sheet is brought into contact with the surface of the object and produces a 2D mapping of the distribution of elements that give certain chemical reactions, precipitates, or color (Fig. 17b). Photographic paper impregnated with diluted sulfuric acid as etching reagent is used to reveal an imprint of the sulfide segregations in steel alloys [49]. This technique was also conducted using filter paper or gelatin-coated paper [50]. Although simple, this method has limitations such as restrictions in the choice and use of the etching reagent, low control of the process of solution, and further diffusion of the dissolved ions into the printing medium. 


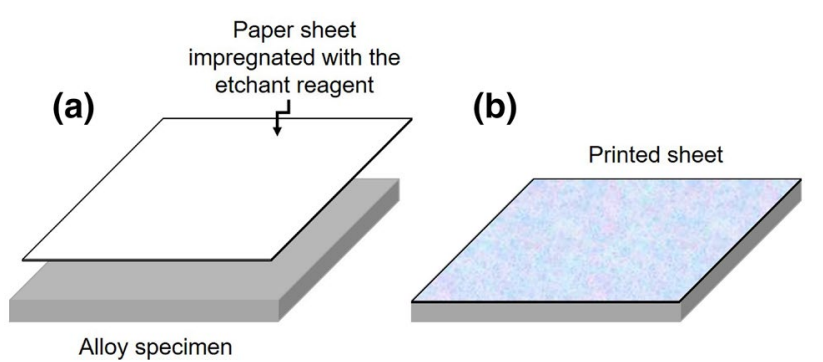

Fig. 17 Arrangement of the chemical contact printing test

\section{Electrographic testing}

The electrographic test, proposed by the Russian chemist Aleksandr Il'ich Glazunov [50] in 1929, is based on the electrolytic dissolution of surface constituents and their diffusion into an attached electrolyte-containing paper. The electrolytic solution of the metal specimen (or ore, mineral) can be achieved by connecting an external voltage source between a metallic plate that acts as cathode and the anodic specimen. In the simplest case, a paper impregnated with an inert electrolyte and a suitable reagent is sandwiched between the inert metal plate and the flat surface of the specimen. Both the plate and specimen are connected externally to an outside voltage source (Fig. 18a) so that the ions released from the specimen move into the paper where they react with the reagent and form a colored product. Thus, the selective dissolution of ions from the surface of an alloy can be achieved. Usually, the specimen acts as anode and the metal plate as cathode. Occasionally, the species to be recognized are anions and the specimen acts as cathode. Sometimes, the external source of potential can be suppressed and then the metal plate and the specimen are externally connected by a low resistance conductor: the galvanic cell formed in this way is activated as a result of the internal spontaneous electrolysis (Fig. 18b). In all cases, a rather uniform electrical field is formed between the two flat electrodes resulting in an orthogonal movement of the released ions, avoiding lateral diffusion of the ions along the paper. The reagent directly fixes the ions in the paper and provided a high-fidelity image of the 2D distribution pattern of the ions in the specimen.
The electrographic testing was mainly used to reproduce the granular distribution of phases of an ore, metal, or alloy in the form of an imprint (Fig. 19).

Sometimes, the formation of by-products due to undesirable interfering reactions does not allow one to place the metal plate in direct contact with the flat specimen with only the paper between. This problem was solved by placing the paper sheet on a glass plate and over an outer ring electrode around the specimen (Fig. 20).

Alternatively, specimens with small flat surfaces were tested with test probes. Specimens with irregular shape or others, like wires and pins, were held in the hand or in jewellers' pins orienting them on the paper. In such instances, electrical external connection was made with alligator clips. For large specimens or immovable objects,

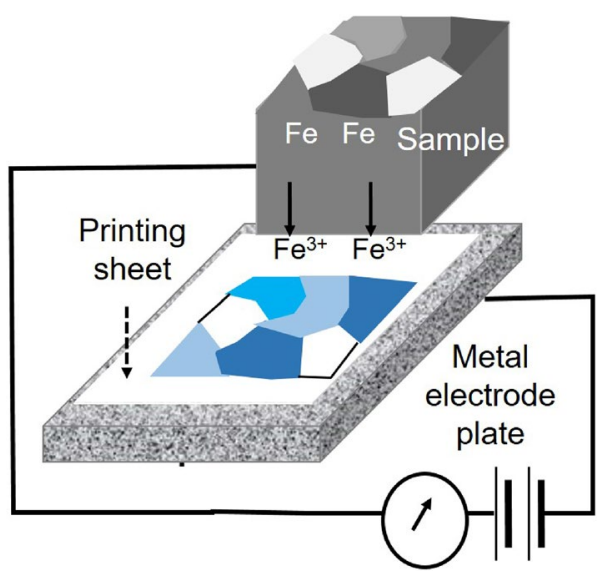

Fig. 19 Scheme of the experimental arrangement of the electrographic test by Glazunov. When the process is finished and the specimen is removed, the printed distribution pattern can be seen on the paper

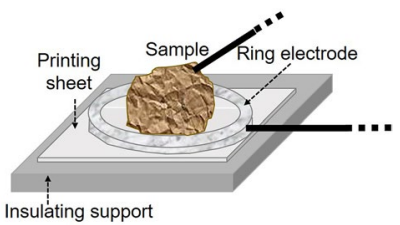

Fig. 20 Arrangement using an inert ring electrode
Fig. 18 Scheme of electrotransfer method: a arrangement with potential outside source; b arrangement with internal electrolysis (galvanic cell)

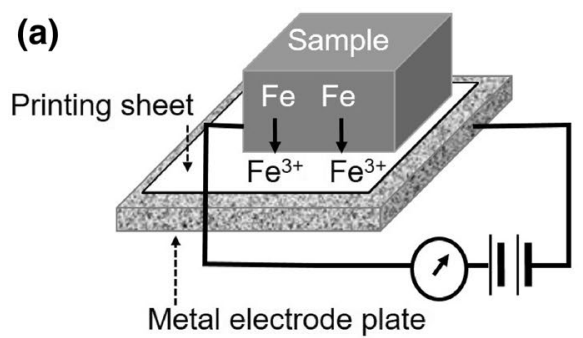

(b)

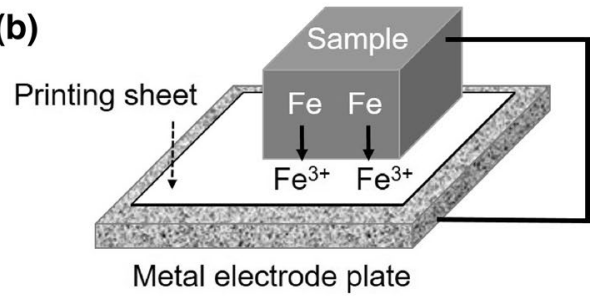




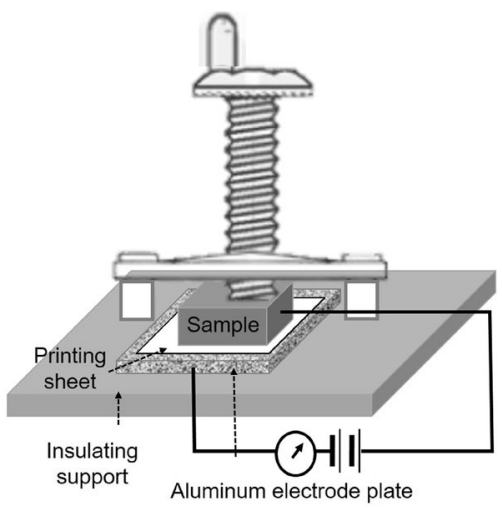

Fig. 21 Arrangement including a press for the control of pressure

spotting electrodes were used that contact the test area of the object, covered with the impregnated paper. For a high-fidelity reproduction of the distribution pattern of the surface of the object, accurate regulation of the pressure applied to the "specimen-paper-metal plate" system was required. Several methods were developed that allowed the control of a uniform, high, and stable pressure during the electrographic process. One of the simplest arrangements is shown in Fig. 21.

Simultaneous imaging of a large number of samples was accomplished with the arrangement shown in Fig. 22. The mounting block includes a number of pellets $18 \mathrm{~mm}$ long and $12 \mathrm{~mm}$ diameter of different materials that are inserted in a block with holes adapted to the size of the pellets and a spring in the bottom of the pellet for controlling the pressure applied on the system. When several electrographic imprints are made under analogous electrolysis conditions, the imprints can be easily compared (cf. Fig. 22). For this aim a series of pellets made with the same alloy, but containing different known amounts of the element to be analyzed are prepared and simultaneously analyzed with the pellet of the unknown material. The concentration of the analyte is calculated by comparing the color density of the imprint.

In electrographic testing the external electric circuit must include a series of parts: a battery to supply the suitable voltage with a polarity switch for changing the direction of the current through the printing cell, rheostats in series for constant and suitable currents, a milliammeter preferably covering 1 to $500 \mathrm{~mA}$, a voltmeter for 0 to $10 \mathrm{~V}, 1000 \Omega \mathrm{V}^{-1}$ and, optionally, a timing switch, if calculating the quantity of dissolved ion is required. The current and time for printing vary with the area and nature of the specimen, which determine the amount of ions transferred to obtain an appropriate print density according to Faraday's second law. Values in the range $20-70 \mu \mathrm{g} \mathrm{cm}^{-2}$ of electrolyzed ions are commonly used. Nevertheless, the current density should be selected to ensure that the anodic voltage does not exceed the decomposition potential of the analyte. Pressure applied to the system varies depending on each case. Pressures of $0.69 \times 10^{6} \mathrm{~N} \mathrm{~m}^{-2}$ to $0.17 \times 10^{6} \mathrm{~N} \mathrm{~m}^{-2}$ were commonly used but could increase up to $1.08 \times 10^{6} \mathrm{~N} \mathrm{~m}^{-2}$ if a hydraulic press was used.

Similar to common photography, electrographic imprints should allow for a better spatial resolution than the distribution of the details of the specimens. Therefore, the transfer medium (mostly paper) must accomplish a series of characteristics: a structureless material, transparent if possible, conductor of the electric current, resistant to the action of the chemical reagents, and exhibiting minimum distortion while drying. Bubbles must be avoided in the printing medium. Although commercial sized and filled papers such as heavy grade copying paper yielded prints with great details, gelatin-coated paper handcrafted or commercially supplied as "imbibition paper" was preferred owing to the high-quality prints. For microscopic observation, prints with transparent base of cellophane or plates of plaster of Paris $1 \mathrm{~mm}$ thick were used. This last material was also used when objects with irregular shape were analyzed.

Inert metals with scarce reactivity such as aluminum or steel were used as opposite electrode. Occasionally, if the object had irregular shape, thin foils of aluminum or tin were used as well as melted alloys such as Wood's metal. It must be taken into account that the electrode surface has to be cleaned frequently in order to avoid tarnish and deposition of electrolysis products that can cause contamination resulting in false distributive patterns.

Printing reagents depend on the metal to be identified. In some cases, direct printing was possible as for iron using potassium hexacyanoferrate(II), which reacts with diffused $\mathrm{Fe}^{3+}$ ions to form iron(III) hexacyanoferrate(II) (Prussian blue) [51]. A contribution by Kraft dedicated to Prussian blue can be also found in ChemTexts [52]. In other cases,

Fig. 22 Scheme of the standard specimen block
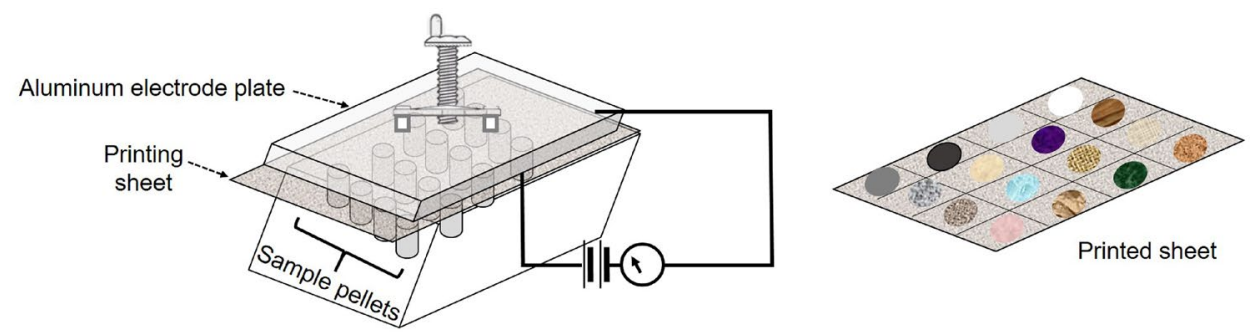
an indirect method was used in which the paper sheet that contains the metal ions is taken out of the printing arrangement and subjected to further operations with one or more reagents until the color product is obtained. For example, identification of manganese can be conducted by transferring the metal ions present in the metal or alloy specimen as sulfates. Then, the print is treated with ammonia and hydrogen peroxide to transform the manganese salt into hydrated manganese dioxide. Finally, the print sheet is treated with benzidine acetate so that the blue oxidation product of benzidine produces an image of the manganese. This procedure enables the separation of interferents such as copper, which is removed by washing.

The selection of the electrolyte was made considering that the role of this solution is to provide suitable conductivity between the two electrodes and through the interposed printing medium. Salt concentrations in the range of $0.2-0.5 \mathrm{~mol} \mathrm{~L}^{-1}$ are commonly used. The electrolytes containing color-forming ions suitable for direct electrographic printing are rather limited: iodide, sulfide, chromate, hexacyanoferrate(II) and (III), and nitrite are the most common. For this reason, electrolytes of general use are employed in combination with specific color-producing reagents. The $\mathrm{pH}$ plays an important role during the electrographic process when a neutral salt is used as electrolyte as the $\mathrm{pH}$ varies continuously through the printing sheet from acidic values at the anode to basic values at the cathode.

The preparation of the specimen varies according to the aim of the analysis. For the identification of inclusions and composition of the surface of an ore, metal, or alloy, a gentle treatment with organic solvents to remove dust and lipids deposited on the surface is enough. For structural studies of the material abrasive treatments are commonly used. Small objects can be directly treated with abrasive $\mathrm{SiC}$ powder or sheets, with alumina emulsion or diamond paste to obtain a polished and clean surface. For large site objects, a metallographic treatment is necessary. Currently, in the metallographic procedures the sample is embedded in a hardening synthetic resin (epoxy, polyester, acrylic) using silicone or PVC molds followed by wet polishing with $\mathrm{SiC}$ abrasive disks, alumina emulsion, or diamond paste applied with a felt.

\section{Spot tests in practice: past and present of this classical method}

In the late twentieth century a variety of novel analytical methods emerged, whose roots lie in the principles of the classical spot tests and have achieved improved fastness, sensitivity, specificity, ease of use, portability, and sustainability. Undoubtedly, the development of advanced spot testbased methods such as immunospot tests, immunoassays
(IAs), microfluidic devices, and multiplexed assays has been promoted by the rapid development of innovations in nanotechnologies, functionalized materials, three-dimensional (3D) printing, microchips, or smartphone spectroscopy, among others. For this reason, the first part of this section is devoted to describing these novel spot test-based methods and some of the advanced technologies that are boosting their development. The historical evolution of the spot tests in the more important application fields is also reviewed in the last subsections.

\section{Advanced technologies boosting innovations in spot testing}

The incorporation of nanotechnologies into the field of analytical chemistry has had an important impact on the development of novel analytical methods. On the other hand, the dramatic popularity of mobile phones has led to an advanced generation of instruments, the smartphones, with great capability and versatility; this has converted them into almost portable computers and given rise to a new science, smartphone spectroscopy, with interesting applications in analytical chemistry. These new technologies have contributed to the development of innovative and sophisticated spot testbased methods.

\section{Nanomaterials}

Nanotechnologies are manipulations of materials at the nanoscale for creating new nanoparticulated materials with special shapes at molecular scale (i.e., a unique dimension or large surface area to volume ratio) that improve their properties and increase their reactivity. Some advantages of nanoparticles (NPs) are their cost-effectiveness and time-saving and eco-friendly character. Among other applications, NPs play an important role as components of detection systems in which the NPs participate actively in the chemical, biochemical, or physicochemical process of detection at nano- or microscale by enhancing signals. Among the nanomaterialbased detectors (sensors) ${ }^{7}$, stand out those applied in IAs and microfluidic devices or devices combining both owing to their inexpensive, quick, sustainable, highly sensitive, selective, reliable, and easy-to-use character. In these techniques, the detectors are composed of two components: a chemical (molecular) or immunochemical recognition system (receptor) and a physicochemical transducer. In classical spot tests

\footnotetext{
${ }^{7}$ IUPAC has defined a chemical sensor as: "a device that transforms chemical information, ranging from concentration of a specific sample component to total composition analysis, into an analytically useful signal”. Hulanicki A, Gląb S, Ingman F (1991) Pure Appl Chem 63:1247-1250.
} 
the receptor is the reagent system yielding an end-product whereas in IAs an affinity-ligand-based solid-state arrangement is formed in which the NPs, directly or indirectly, link the target species forming a labeled analyte-containing complex species. The transducer connected to the receptor generates a readable signal from the labeled complex. Four principal classes of transducers are employed in IAs: optical, electrochemical, piezoelectric or mass sensitive, and magnetic [53]. A contribution by Gründler dedicated to chemical sensors can be found in ChemTexts [54].

Optical detectors are popular owing to the rapid signal generation and reading based on the irradiation of the immunosystem with light. Optical detectors are classified according to the phenomenon involved in surface plasmon resonance and luminescence detectors.

The most employed labeling strategy is the colorimetric detection based on the ability of NPs to exhibit a different color after attaching to the analyte complex. The signal readout is carried out with the naked eye in the simplest tests or using a colorimetric digital or spectrophotometric detector in more sophisticated tests [53].

Luminiscence is the property of the label NP to directly or indirectly induce an emission of light that is used as signal. Three main luminescent detections are used depending on the mechanism employed: photoluminescence, chemiluminiscence, and electrochemiluminiscence [53].

In photoluminescence, the end-product of the reactive test process re-emits radiation coming from an external light source as a consequence of the transition of this species to an excited electronic state and further decay to the ground state. Fluorescence is the form of photoluminescence most extensively applied. The fluorescent signal can be generated by several NP labels: dye surface-functionalized NPs, metallic NPs, and quantum dots (QDs) (see hereafter). In tests based on metallic NP clusters, one uses the change in intensity of the fluorescence signal that is deactivated by the resonance transfer of fluorescent energy between the clusters of metallic NPs and the analyte (e.g., AuNPs and some bacteria). The quenching ${ }^{8}$ ability of AuNPs is used in some IAs to improve the sensitivity [53].

Chemiluminescence (CL) IAs are based on the ability of NPs to act as catalysts and promote the transformation of a chemiluminiscent substrate (e.g., luminol) to an excited end-product, which emits photons of light as it decays to a lower energy level. The specific luminescent signal detected is characteristic of a specific analyte [53, 55-57].

Electrogenerated chemiluminiscence or electrochemiluminiscence (ECL) is a particular type of chemiluminiscence in which the chemiluminiscent reaction is preceded by an

\footnotetext{
${ }^{8}$ Quenching is a term used to refer to any process in which the fluorescent signal from a substance is decreased.
}

electrochemical reaction. The electrochemiluminiscence sensor includes an external circuit that applies an electrical potential on an electrode (transducer) in contact with the reactant system. This potential initiates an exergonic electron transfer reaction on the surface of the electrode, where an activator analyte or a co-reactant promotes the transition of a label or luminophore to excited states in a series of redox steps as a result of which light (IR, visible, or UV) is emitted. The intensity of the resulting light depends on the analyte content. ECL tags are required in IAs where the target biomolecules are not ECL-active. Luminol and its derivatives, QDs, and other nanomaterials are used for this purpose [53, 58].

Electrochemical detectors use suitable labels that amplify the change in the electrochemical signal due to the immunoreaction. Typical labels are bioactive enzymes, electroactive metal ions, and nanostructures such as metal NP, carbon nanotubes (CNTs), and QDs [53].

The piezoelectric detectors are quartz crystal microbalance devices that measure the mass changes during the IA by the frequency changes induced in the piezoelectric quartz crystal. AuNPs are used for the amplification of the signal [53].

Magnetic NPs (MNPs), when conjugated to antibodies in an IA, form a dark precipitate easily detectable with the naked eye. In addition, they yield a signal with low background that can be measured using a small-scale magnetic reader [55].

A variety of NPs and nanomaterials have been developed with specific properties that depend on their nature and morphology. Additionally, more specific properties and behavior of the NPs can be obtained by functionalization (compositional surface modification) and immobilization by physisorption, affinity interaction, covalent conjugation, or entrapment in sol-gel matrices [53]. As previously outlined, photoactivity is an intrinsic property of certain NPs due to their composition that makes them suitable as lightresponsive receptors. A contribution by González-Carrero and Pérez-Prieto dedicated to photoactive NPs can be found in ChemTexts [59]. According to the properties and structure exhibited by the NPs, several groups can be established [53, 55, 57, 59-66]:

- Colloidal metallic NP, especially AuNPs ( $\varnothing \sim 1-150 \mathrm{~nm})$, exhibit a distinct color. Since the 1980s, this ability has been applied for colorimetric detection in IAs and several strategies have been developed for enhancing the signal: Modification of size and shape (nanospheres, nanorods, nanoshells, and nanostars). Laser excitation induces signal enhancement due to the surface plasmon resonance effect (SPR). SPR is the property for photoinducing collective electron charge resonant oscillations of the conduction electrons in a NP when it is irradiated 
at the resonance wavelength. The absorption of radiation at the resonance range of wavelengths results in a change of the color in the NP that thus acts as a label. The intensity and hue of the detection signal induced by the label NP depend on the shape, size, geometry, surface cover, or the dispersion degree. For example, AuNPs at ca. $30 \mathrm{~nm}$ diameter absorb blue-green light at ca. $450 \mathrm{~nm}$ and re-emits light at ca. $700 \mathrm{~nm}$, which results in a red color. This phenomenon is used in the technique named thermal contrast amplification (TCA). Surface enhanced Raman spectroscopy (SERS)-active AuNPs are hollow or flower shaped so that the SPR effect, which is localized in the nano-gaps of the surface, enhances the Raman signal that is measured with a Raman spectroscope detector. AuNPs are also used in chemiluminiscent and piezoelectric detectors and gold nanorods (AuNR) in electrochemiluminiscence detectors [53]. Amplification can be obtained by surface modification forming a core-shell NP (i.e., $\mathrm{SiO}_{2}$ or polyethylene glycol). Silver- and goldbased amplification is based on the reduction of silver or gold ions by a reducing agent at the place of the AuNPs that act as centers of nucleation of the metallic crystals, which results in a signal amplification [55, 59]. Nanoclusters (AuNCs) are AuNPs with dimensions smaller than $2 \mathrm{~nm}$, so that they adopt discrete electronic structure and exhibit electron energy quantization. AuNCs are fluorescent labels with large Stokes shift [59].

In certain kind of biosensors the label NPs are attached directly to the microorganisms. An illustrative example of this particular detection strategy is the work of Rotello et al. [67, 68], who have developed a nanomaterial-based detector for rapid detection of bacteria in water. AuNPs functionalized with a cationic surfactant are attached to molecules of $\beta$-galactosidase, so that the enzyme is covered with the AuNPs and, consequently, is reversibly inhibited (see Fig. 23). This NP-enzyme complex and the chlorophenol red- $\beta$-D-galactopyranoside colorimetric substrate of the enzyme are inkjet-printed on the pad of a dipstick forming a frame. When the dipstick is in contact with the sample, the positively charged AuNPs, which are released from the enzyme molecule, bind to the negatively charged surface of the bacteria present in the contaminated water. In parallel, the enzyme molecules retrieve their activity and cleave the yellow substrate to yield an intense purple color. The detection of Salmonella typhimurium in drinking water is carried out by the aggregation of antibody-conjugated AuNPs on the surface of the bacterium, which produces a visible color change with sensitivity highly dependent on NP shape [61].

- Quantum dots are colloidal semiconductor NPs ranging from 2 to $10 \mathrm{~nm}$ in diameter of element of groups II-VI (CdSe, CdTe, CdS, ZnS, ZnSe), III-V (GaAs, GaN, InP,
InAs), and IV-VI (PbS, PbSe, PbTe) with a spherical shell. This structure induces quantum confinement in the movement of the electrons of the continuous conduction band that, in turn, acquires a discrete energy level structure. The new electronic configuration gives fluorescence. The emitted light can be tuned by selecting the composition, shape, and size of the NPs. Different-sized QDs can be excited by single wavelength light sources and the emission spectra are tuned resulting in narrow emission profiles [53, 55, 59]. Since 2010s, QDs can be prepared as nanobeads (QBs) or synthesized by embedding single QDs (i.e., $\mathrm{CdSe} / \mathrm{ZnS}$ ) into a polymer matrix [62, 63].

- Porous silica NPs are used as biocompatible labels owing to their semiconductive properties, water dispersibility, and surface functionality. AuNP-decorated silica nanorods (AuNP-SiNRs) are used as visual labels. Labels can be made by embedding pitaya-type porous silica with high-density QDs (i.e., CdTe QDs). The pitaya-type silica spheres are made by forming pores in a process of mercaptografting followed by embedding different colored QDs into the pores and encapsulating the NPs with a dense organosilica shell for protection. Other NPbased labels yielding photoluminscent signals are luminescent dye surface-functionalized silica and polystyrene NPs (dye-doped) and organic compound microspheres that induce fluorescent emission by aggregation [55].

- Upconversion NPs (UCNPs) are fluorescent labels prepared from NPs made of an inert host matrix such as $\mathrm{NaYF}_{4}$ or phosphor doped with lanthanide ions such as $\mathrm{Eu}$ and $\mathrm{Yb}$ (i.e., doped $\mathrm{NaYF}_{4}: \mathrm{Yb} / \mathrm{Eu}$ ) or other rare earths. The UCNPs exhibit photon upconversion in which an exciting low energy photon (i.e., near-infrared (NIR)) is absorbed in a non-radiative process by the UCNP and converted into other photon of higher energy (i.e., UVvisible) $[55,59,64]$.

- Time-resolved fluorescence NPs (TRFNPs) are La-doped NPs modified with chelates containing bifunctional groups. This structure confers these NPs a fluorescence decay time longer than that of conventional NPs (at ca. $10^{3}-10^{6}$ times), which makes time resolution feasible. In addition, the signal produced has high sensitivity and low background due to the large Stokes shift and broad excitation light spectrum of the fluorescence emitted. Common lanthanide chelates are made with europium $(\mathrm{Eu})$, terbium (Tb), samarium (Sm), and dysprosium (Dy) [55].

- Nanozymes are metallic NPs such as PtNPs or core-shell Pd-PtNPs used as chemiluminiscent labels owing to their enzyme-like activity that mimics peroxidase enzymatic activity. Hybrid label signal enhancement is obtained with Pt-coated AuNPs, which have peroxidase-like catalytic activity while AuNPs maintain their plasmonic activity resulting in a dual amplified signal $[62,65]$. 


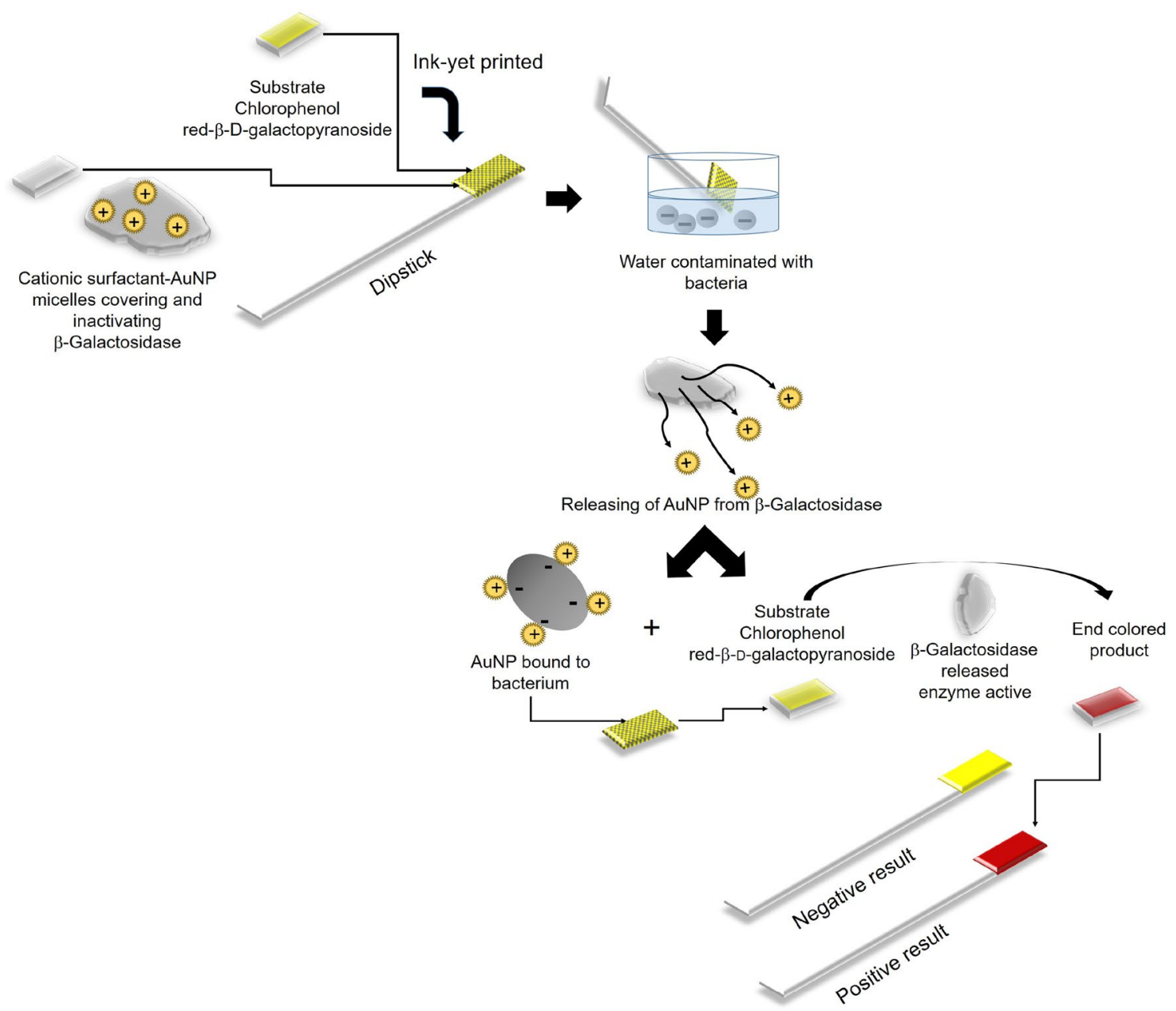

Fig. 23 Scheme of the chemical and biochemical processes involved in the nanomaterial-based detector with inkjet-printed dipstick format used for identifying bacteria in water. Adapted from $[67,68]$

- Magnetic NPs (MNPs) are used as color, chemiluminiscent, and magnetic labels. MNPs are mainly made with $\mathrm{Fe}_{3} \mathrm{O}_{4}$. Other MNPs are made as $\mathrm{Fe}_{3} \mathrm{O}_{4}-\mathrm{Au}$ core-shell NPs, Au-coated MNP complex, or Au magnetic bifunctional NPs [55, 57, 62].

- Carbon nanomaterials include carbon NPs (CNPs) and graphene (GR) combined with silica NPs. These NPs have been used as labels owing to their excellent mechanical, electrical, and chemical properties. Currently, carbon nanotubes (CNTs) are made of hexagonal grid graphite in their two structures,single-walled carbon nanotubes (SWCNT) and multiwalled carbon nanotubes (MWCNT). CNTs possess good stability, easy preparation, and are eco-friendly materials. CNTs can absorb many biomolecules and hence are suitable biosensors of microbial pathogens owing to their special properties such as high surface area-to-volume ratio. That promotes the change of the surface shape by the attachment of bacteria, which yields a higher signal-to-noise ratio than the former planar structures. In addition, the fast electron conductivity enables quantitation to be performed by simple membrane-resistance measurement. Carbon nanomaterials, in general, are used in electrochemical labeling. The development of NIR labels for photoluminescent detection profits from the intrinsic photoluminescence of SWNTs. GR QDs are used in electrochemiluminiscence sensors [66]. Magnetized multiwalled carbon nanotubes (MMWCNTs) can be prepared by deposition of magnetite NPs on MWCNTs. These NPs are used as labels in IAs. The magnetization enables a suitable separation of the MWCNT-labeled analyte using an external magnet, which results in an enhancement of the sensitivity of the signal. The detection is achieved by observing with the naked eye the characteristic brown color of the labeled complexes formed [55, 69].

CNTs can also act by establishing synergies with metals and organic/inorganic materials to enhance the sensitivity of immunosensors. For this purpose they are 
Table 3 Common applications of NPs used in IAs [53, 55, 57, 60-66]

\begin{tabular}{ll}
\hline Type of detection & Nanoparticles \\
\hline Colorimetry & Colloidal metal NPs (AuNPs (spherical and rose-like), AgNPs, SeNsP, etc.), AuNP-SiNRs, AuNCs, latex beads, \\
& PtNP nanozymes, core-shell Pd-Pt NPs, MNPs, COFs, MOFs, CNPs, MSNPs, CNTs \\
Photoluminiscence & $\begin{array}{c}\text { Luminescent dye-doped (surface functionalized) silica and polystyrene NPs, CdTe QDs in silica NPs, porous silica } \\
\text { of pitaya-type with high-density QDs, AuNP-SiNRs, UCNPs, lanthanide chelate NPs such as Eu, Tb, Sm, and }\end{array}$ \\
& Dy chelate NPs, single- and multi-shell (CdSe-ZnS) QDs, core-shell CuInZn $\mathrm{S}_{2+\mathrm{x}} / \mathrm{ZnS} \mathrm{QDs,} \mathrm{biotin-QD-MnO}_{2}$, \\
& Mn-doped ZnS QDs, graphene QDs-AgNPs, chitosan-derived carbon dot-CdTe QD pairs, organic compound \\
& microspheres, chelated MSNPs, SWCNTs, COFs, MOFs \\
Chemiluminiscence & AuNPs, UCP labels, MNPs, CNTs \\
Electrochemiluminiscence & Graphene QDs, CNTs, AuNRs \\
Electrochemical & CNTs, SWCNTs, MWCNTs, QDs, metal NPs \\
Piezoelectric & AuNPs \\
Magnetic signal & Magnetic loaded NPs (MNPs): gold- and CNT-coated MNPs such as Au-Fe $\mathrm{O}_{4}$ core-shell-NPs, Fe $\mathrm{O}_{4}$-core and \\
& SiO ${ }_{2}$-shell NPs, poly(acrylic acid) (PAA)-modified gold magnetic NPs, AuNP-coated starch magnetic beads, \\
& gold magnetic bifunctional NPs
\end{tabular}

coupled with metals and oxides such as $\mathrm{Fe}_{3} \mathrm{O}_{4}$ (colorimetric, photoluminescent, and electrochemical), $\mathrm{ZnO}$ (electrochemiluminiscent, photoluminiscent), AuNPs (colorimetric, electrochemical), and SWCNT-chitosan and MWCNT-polystyrene (electrochemical). A variety of electrochemical platform IAs have been developed using (a) multilayer CNTs combined with other metal NPs and materials such as MWCNTs-chitosan and platinum-Prussian blue hybrid NPs, (b) CNTs incorporated with mesoporous silica (MPS) and GR, and (c) CNTs incorporated with ionic liquid (IL) and fullerene $\left(\mathrm{C}_{60}\right)$ [66].

- Metal organic frameworks (MOF), covalent organic frameworks (COF), and mesoporous NPs (MSNPs) have been used as support of labels in several colorimetric and fluorescence detection procedures [62].

Table 3 summarizes some of the most common detection strategies and the corresponding nanomaterials used in IAs $[53,55,57,60-66]$.

\section{Cellphone technologies}

The mobile phone or cellphone (CP) was devised originally as an apparatus for portable communication in the 1990s. Since then, advances in artificial intelligence algorithms and electronics led CPs to improvements in the direction of a personal microcomputer-like platform. Currently the new generation of CPs, the smartphones, are equipped with multi-core processors, touchscreens, cameras, large capacity batteries, and other common components. However, the range of applications can be extended to other fast optical imaging systems and devices such as accelerometers, hygrometers, scanners, and heart rate sensors. In parallel to the conventional wireless communication, smartphones can also transmit data between individuals or to the cloud. All that opens the possibility to collect and process data via external attachments and third-party applications. Therefore, smartphones have become robust portable instruments that require minimal training. The possibility of using CPs as an independent platform that provides fast optical measurements with the built-in camera and app without other accessories has resulted in their progressive application in both classical and advanced spot testing. Fields such as food, forensic, clinical, and environmental analysis, where on-site analysis in crime scene, point-of-care (POC), or monitoring in real time is required, have exponentially increased the number of methods in which this technology has been implemented [70, 71].

According to Chen et al. [70], the term smartphone spectroscopy "refers to the process of taking images using the built-in optical camera and then analyzing the images with additional attachments or applications provided by a third party". Those same authors establish three types of camera or sensor systems based on the type of light signal processed: micro-imaging, colorimetric, and luminescence. In the following, the focus of consideration is put on the description of these sensors. In addition, smartphones can be used as electrochemical sensors [71].

The latest generation of smartphones have built-in dual cameras, which contain contact image sensors (CIS) made with complementary metal-oxide semiconductors (CMOS) and equipped with microlens and color filters. ${ }^{9}$ Alternatively,

\footnotetext{
9 There are two main types of image sensors: the CIS and the chargecoupled device (CCD). The former is mainly used in CPs and flatbed scanners owing to its smaller size. The CIS device is composed of focusing lens, a linear array of detectors, and LEDs as light source, which confers the CIS a high power efficiency. Usually power supply is covered via USB connection.
} 
(a)

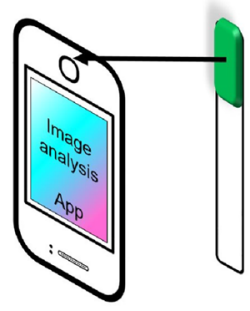

(b)

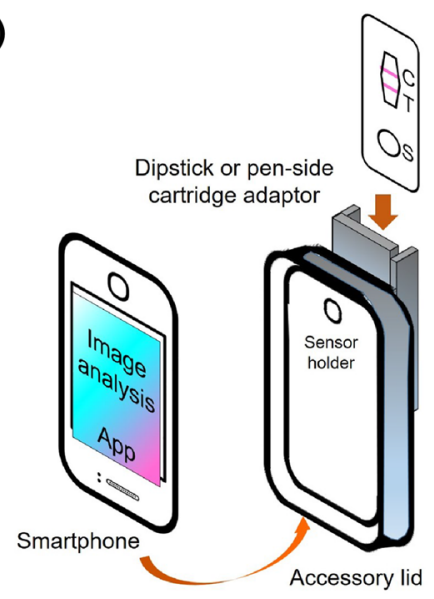

(c)

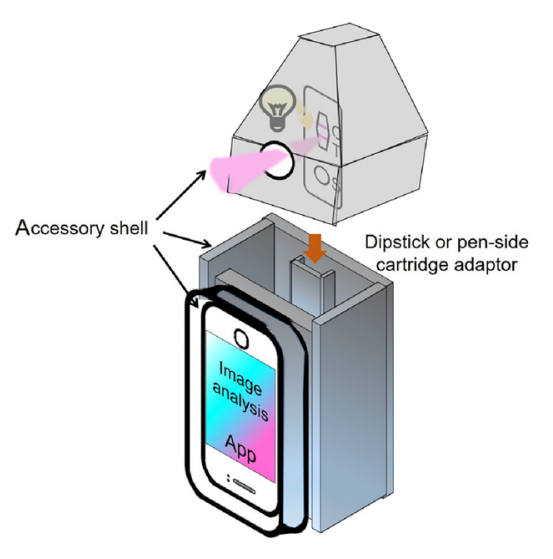

Fig. 24 a Scheme of the simplest smartphone colorimetric device; b customized smartphone colorimetric device with a smartphone lid and cartridge adaptor for the dipstick or pen-side device; c custom- ized smartphone device with holder, cartridge adaptor, and accessory shell for photoluminescence detection

taken with the digital camera, the color filters of the CIS Bayer mosaic provide the intensity color of each pixel in the digital image. ${ }^{10}$ The raw data of the pixel grid that conforms with the digital image are stored in the smartphone so that they can be processed further. A simple approach consists of measuring the change of reflectance of the strip or plate at the end of the test, which enables quantitative analysis provided that a prior calibration is performed. The values obtained can be processed into signal values related to absorbance and, therefore, Lambert-Beer's law can be applied. A contribution by Scholz and Scholz as well as by Oldham and Parnis on first-order differential equations and Beer's law, respectively, can be found in ChemTexts [72, 73]. Nevertheless, the most frequent method for determining the analyte concentration is based on measuring the change in the color yielded in the test. Red-green-blue (RGB) values but also hue-saturation-brightness (HSB) or hue-saturation-value (HSV) are used. Color values as well as pixel, color, and luminous intensity ${ }^{11}$ can be obtained by specific apps for

\footnotetext{
10 The Bayer filter mosaic is a color filter array (CFA) formed by a square grid of photosensor cells outfitted with alternated filters of the three primary colors RGB in different proportion, $25 \% \mathrm{R}, 50 \% \mathrm{G}$, and $25 \%$ B. The Bayer mosaic is placed on the photosensor of the CIS. This array enables one to yield a color image formed by the grid of pixels with only one sensor. The resulting color in each cell or pixel is calculated by a process of demosaicing based on the interpolation of the RGB values of the neighboring cells. The digital matrix of light intensity $(I)$ stores each R, G, B color value in 256 levels in a scale from 0 to 255 , where 0 is pure black and 255 is pure white. Thus, each pixel has a color characterized by the $I_{\mathrm{R}}, I_{\mathrm{G}}$, and $I_{\mathrm{B}}$ combination of values.

11 Luminous intensity measured in candelas (cd) is defined in photometry as the wavelength-weighted power emitted by a light source in a specific direction per unit solid angle. The SI unit is the candela (Sears FW (1971) Fundamentos de Física III. Óptica (Fundamentals of Physics III. Optics), Aguilar, Madrid).
} 


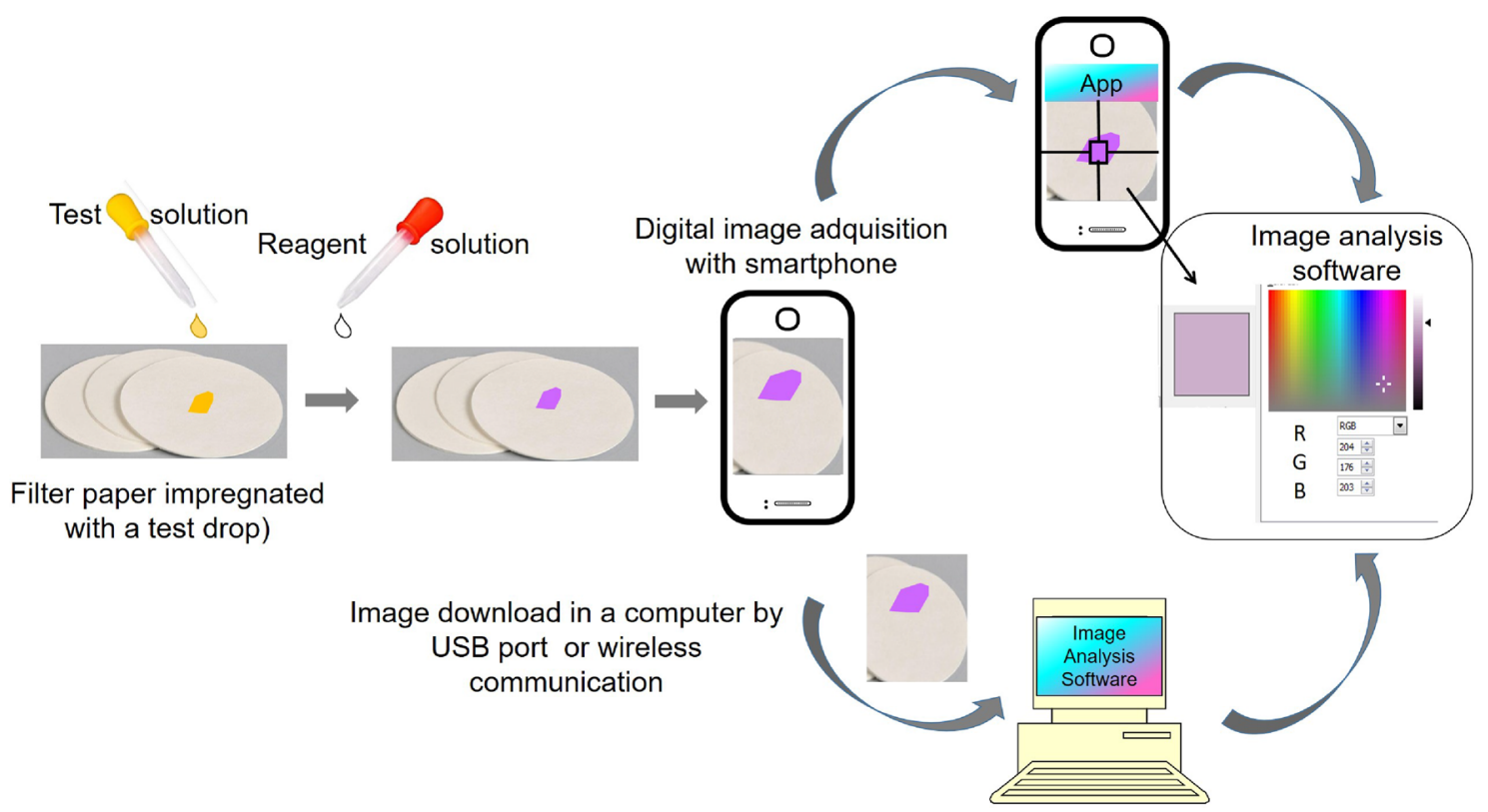

Fig. 25 Scheme of the process of spot test combined with digital image acquired with a smartphone camera and processed with smartphone image analysis app. Alternatively, the image is downloaded from the smartphone in a computer via USB port or wireless communication

color analysis such as PhotoMetrix, ImageJ, AdobePhotoShop, Touch Color, Color Picker, MatLab, and Color Grad compatible with iOS or/and Android operating systems or by means of self-developed apps. Alternatively, the images acquired with the smartphone are download into a computer equipped with image analysis software via USB port or wireless communication (Fig. 25). The digital sensing information obtained can be saved on cloud servers, pushed on social web media or on an internet map service for public sharing and viewing [70, 71].

Sometimes accessory instruments are adapted to the basic smartphone device for performing DIC, e.g., an optomechanical highly selective colorimetric detection unit coupled to a LED source. A specific app transforms images into binary mask images that are processed into signal values related to absorbance.

Other types of sensors based on luminescence measurements can be implemented in smartphone-based devices: photoluminescence, chemiluminiscence, and electrochemiluminiscence sensors. The simplest fluorescence smartphone platforms include an adapter for a battery-powered laser diode with disposable tubes and filter, sample holder, and back hole. The camera of the smartphone receives the filtered fluorescent signal that is treated in a custom data processor for analysis. More sophisticated appliances can perform simultaneously colorimetry and fluorescence analysis. The smartphone platform for chemiluminiscence detection comprises a cartridge to hold the reactant system and a customized lid or shell adapted to the smartphone with plano-convex lens [70, 71]. Electrochemiluminiscence sensors are mainly implemented in microfluidics technology owing to its reduced dimensions. Paper-based microfluidic electrochemiluminescence devices have three layers: a top transparent plastic layer, a middle layer where the reaction takes place, and the bottom layer with screen-printed electrodes (SPEs) [70, 74]. ECL signals and electrochemical potentials can be controlled by the smartphone via the USB onthe-go (USB-OTG) ports [70].

Advanced smartphones can be connected to electrochemical systems and thus they can be operated as portable electrochemical sensors based on both redox and non-redox reactions. Electron transfer between species (molecules, ions, or particles) is involved in redox reaction-based electrochemical detection. Non-redox electrochemical sensors determine the amount of analyte by measuring the intensity of several electrical parameters. Smartphone-based non-redox electrochemical sensors can be classified into amperometric, potentiometric, and impedimetric $[71,75]$. Electrochemical detection systems can also be used in IAs for antigen detection. Antigens are labeled with antibodies conjugated to oxidizing agents that are then mixed with electron mediators and applied on a working electrode. Detection is based on the oxidation or reduction of electroactive species induced by the application of a specific electrochemical potential between the reference and the working electrode. The entire process is controlled by means of the smartphone platform [76].

\section{Advanced methods of spot testing}

\section{Immunologic analysis}

An important goal in immunology is the identification of the disease in a patient by detection of the presence of a specific antigen 
or toxic substance in body fluids. That has led to development of analytical methods based on the binding of the unknown antigen to its antibody counterpart for its unambiguous identification, which has given rise to analytical immunology or immunomics. Two factors have been essential tools in this methodology: the artificial production of antibodies and the development of methods for recognizing the antigen-antibody couple formation via naked eye or with suitable reading instruments.

\section{Immunospot tests}

Between 1957 and 1963 Joseph G. Feinberg stated the concept of immunospot test for clinical diagnostics by developing the "microspot test" technique, which was initially applied by this scientist to detect autoimmune antibodies and tissue antigens on agar [77, 78] and cellulose acetate membranes [79] in patients with autoimmune thyroid disease. This method, which has been considered by some authors as the first protein micro-spotting technique [80], is a simple and quick microprocedure that enables detection of low levels of either antibody or antigen. The spot test consists of forming a microspot by embedding antiserum in an agar or cellulose acetate thin film placed on a suitable support (i.e., a microscope cover slide). Then the antigens are dropped onto the microspots with pipettes or capillary tubes. After a period of incubation in a moist environment, ranging from $1 \mathrm{~h}$ to 1 day, the presence of an antibody-antigen precipitate indicates a positive test result. The microspot test was later abandoned until 1990 when Ekins et al. [81, 82], inspired by this methodology, developed the multi-microspot IAs. This new generation of immunospot tests was fundamental to the development of multiplex microarrays. Multiplex spot tests and multiplexed immunotests have since become robust spot multianalyte detection methodologies that have experienced a notable growth and expansion (see hereafter).

\section{Immunoassays}

In parallel to the development of immunospot tests, the IAs methodology started to be developed. IAs combine the classical spot test principles with those of biochemistry and immunology. The IA is a process carried out at microscale $(\geq 100 \mu \mathrm{L}$ of sample), in which a target analyte, present in a sample (matrix) such as blood, urine, saliva, sweet, water, beer, milk, juice, etc., binds selectively to a key reagent, the antibody, forming a thermodynamically stable antigen-antibody complex that is detected using a labeling mechanism. The US physicist Rosalyn Susmman Yalow (1921-2011) (Fig. 26) aided by the US physician Solomon Aaron Berson (1918-1972) developed the first immunoassay, a radioimmunoassay (RIA), for detecting human endogenous plasma insulin in 1959 [83, 84]. A radioisotope was used as label for monitoring the analyte. Yalow was awarded the Nobel Prize in Medicine in 1977 for this innovation.

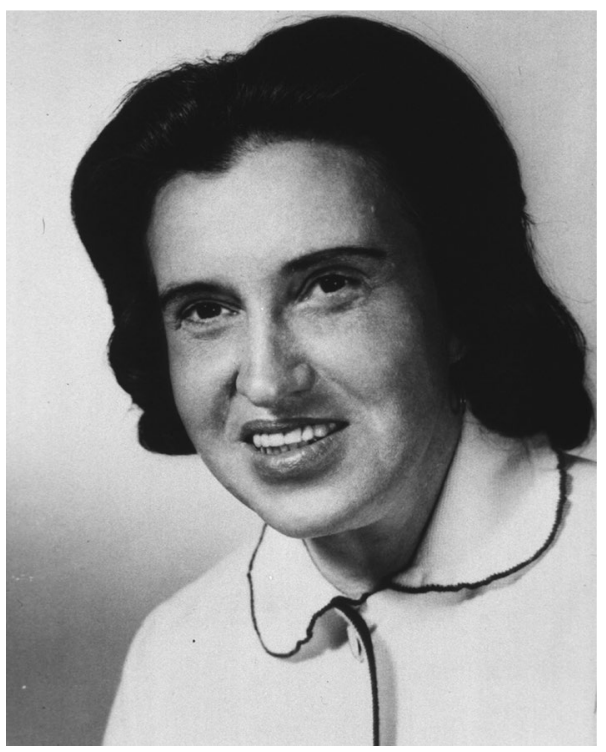

Fig. 26 Rosalyn Susmman Yalow. Photographic collection of the US National Library of Medicine

Since then, nonisotopic immunoassays (hereafter immunoassays, IA) have been gaining general acceptance, mainly because of restrictions in the use of radioactive substances. Moreover, the sorts of target analytes have expanded from protein antigens to other macromolecules or microorganisms as well as small organic molecules (haptens ${ }^{12}$ ) such as a pesticides or drugs.

Nowadays three IAs are by far the most abundantly used: the dipstick immunoassay, lateral flow immunoassay (LFIA), ${ }^{13}$ and enzyme-linked immunosorbent assay (ELISA) [85]. These techniques differ in the IA principles on which they are based (Fig. 27). While ELISAs are enzyme immunoassays (EIAs), dipstick IA and LFIAs are immunomigration assays. Dipstick IA, LFIA, and ELISA have made a fundamental contribution to microanalysis in forensics, life sciences, pharmacology, environmental sciences, industry, and agriculture as well as for the diseases diagnostics in medicine and veterinary [85].

\footnotetext{
12 A hapten is a small molecule (molecular mass $<100$ ) that cannot unaided induce an antibody response. The hapten couples by the amino or carboxyl group to a large carrier protein to yield an immunogen (antibody). Most analytes in the environmental and pharmacological fields are hapten molecules: toxins, drugs, or hormones. Miller JM, Reinhardn N, Knopp D (2001) In: Handbook of Analytical Techniques. (Günzler H, Williams H eds) Wiley VCH, New York, pp 166-168.

${ }^{13}$ LFIA is also called lateral flow immunochromatographic assay (LFICA). LFIA and dipstick IA are generically called rapid strip test (RST), rapid detection test (RDT), express tests, pen-side tests, or quick tests.
} 
Fig. 27 Scheme of the studied IA techniques and the different formats in which they can be performed

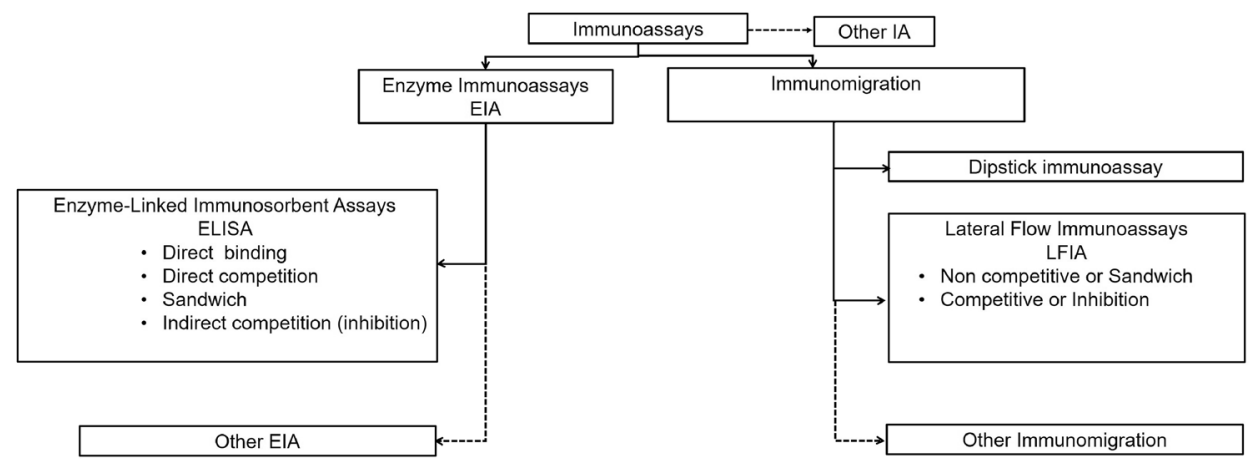

(a) Dipstick immunoassay: This analytical method combines the principles of IAs and those of spot tests conducted in a planar support (paper or membranes). Membrane-based IAs date back to the 1950s and their roots lie in the latex agglutination assays. The advances in manufacturing of nitrocellulose or polyvinylidene difluoride membranes and reagent-dispersing devices promoted the expansion of this new technology in the 1970s. The sample solution containing the target antigen (analyte) is mixed with a reagent solution that consists of specific antibodies attached to a label such as a metallic NP ( $\mathrm{Au}, \mathrm{Ag}$ ) with chromophore properties forming an antibody-NP conjugate. The antigens are bound to the antibody-NP conjugates forming antigen-antibody-NP conjugate complexes (Fig. 28a). The dipstick is immersed in the solution that flows up by capillary forces until it reaches the test pad containing capture antibodies. The capture antibodies, in turn, react with the antigen-antibody-NP complexes and form a colored capture antibody-antigen-antibody-NP complex. The unbound antibody-NP species are captured in the control line by a different capture antibody. A positive test result is indicated by the change in color of both test and control lines, whereas a negative result is indicated by a colorless test line and colored control line showing suitable migration throughout the stick. The readout can be performed with the naked eye or by means of an appropriate reader instrument (Fig. 28b) [85].

(b) Lateral flow test immunoassay (LFIA): The first LFIAs were commercialized in the 1990s and are a technical advancement of the dipstick IA. The scheme of com- mercially available LFIAs is shown in Fig. 29. The device, a few centimeters long, has a window labeled $\mathrm{S}$ (sample) and a second window containing two reactant lines labeled T (test) and C (control) (Fig. 29a). The $\mathrm{S}, \mathrm{T}$, and $\mathrm{C}$ areas contain fixed pads that consist of capillary beds made of thin pieces of porous paper, or microstructured or sintered polymer. The pads have the capacity to spontaneously retain fluids (water, blood, urine, saliva, etc.). For dry solid samples such as blood stains a buffer included in the LFIA kit (buffer small flask + pen-side device) is used to dissolve the dry material or body fluid. The sample pad or the buffer solution can also contain surfactants, proteins, or substances to control the flow rate, among other additives [85]. (a)

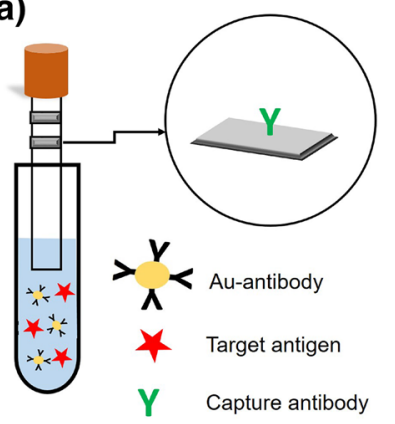

(b)

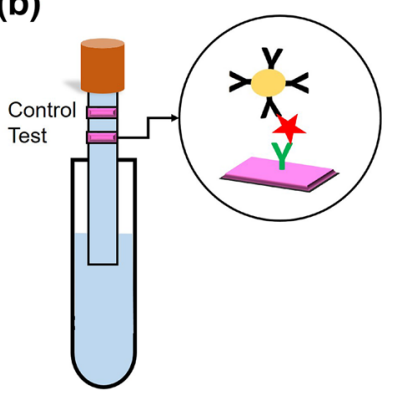

Fig. 28 Scheme of a dipstick IA. a A solution containing the target antigens (analyte) and antibody-NP conjugates; b the dipstick is immersed in the solution that flows up by capillary forces until it reaches the test and control pads containing capture antibodies that react with antigen-antibody-NP complexes in the test line. Unbound antibody-NP are captured by capture antibodies in the control line. Adapted from [85] 


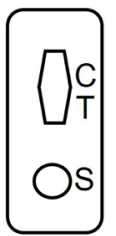

A

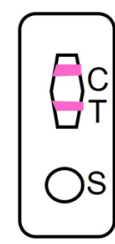

B

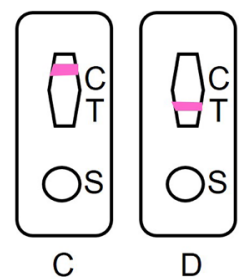

$\mathrm{D}$
Fig. 29 Scheme of the device for the LFIA. a Before sample is poured in the window $S$; $\mathbf{b}$ positive result; $\mathbf{c}$ negative result; $\mathbf{d}$ failed test

LFIAs can be produced in two formats (cf. Fig. 27). Sandwich LFIA is used for detecting high molecular weight molecules with several epitopes ${ }^{14}$ such as proteins and microorganisms. Here the response in the test zone is proportional to the amount of analyte in the sample. A scheme showing how the LFIA in sandwich format works is illustrated in Fig. 30. The dry spot containing the target protein is dissolved with the buffer and poured into the window S pad (Fig. 30a). Then the slurry sample flows to a second pad (the conjugate pad), following the sample pad, where antibodies specific for the analyte (antigen) are immobilized on the pad surface linked to labels such as NPs forming an antibody-NP conjugate (Fig. 30a). The conjugate pad also stores carbohydrates, which act as preservative and resolubilization agents for the antigen-antibodyNP conjugated complex and all the reagents required to optimally produce well-defined results in the test line. Colored complexes formed by an antigen and an antibody conjugated to a chromophore NP, which can be detected with the naked eye, is the most common signal label, but also other signals (i.e., nanozymes, photoluminescent, chemiluminiscent, electrochemical, magnetic, SERS) from suitable conjugated labels can be produced (Fig. 30b). The mobile complexes of antigen-antibody-NP conjugate, the free antibody-NP conjugates, and the rest of the reagents migrate through the absorbent nitrocellulose membrane strip towards a pad or test area $\mathrm{T}$ where a second series of antibodies are fixed, the capture antibodies (Fig. 30c). There a colored sandwich capture antibody-antigen-antibodyNP conjugate complex is formed (Fig. 30d). The free antibody-NP conjugates are linked to the capture antibodies in pad $\mathrm{C}$ forming a colored product that is used as control to indicate correct performance of the assay (Figs. 29b, 30d). Then, the result of the test is considered positive. All the materials that have not reacted are absorbed in the wicking pad, thus preventing backflow

\footnotetext{
14 An epitope is a part or sequence of the macromolecular antigen that binds to a specific antibody.
}

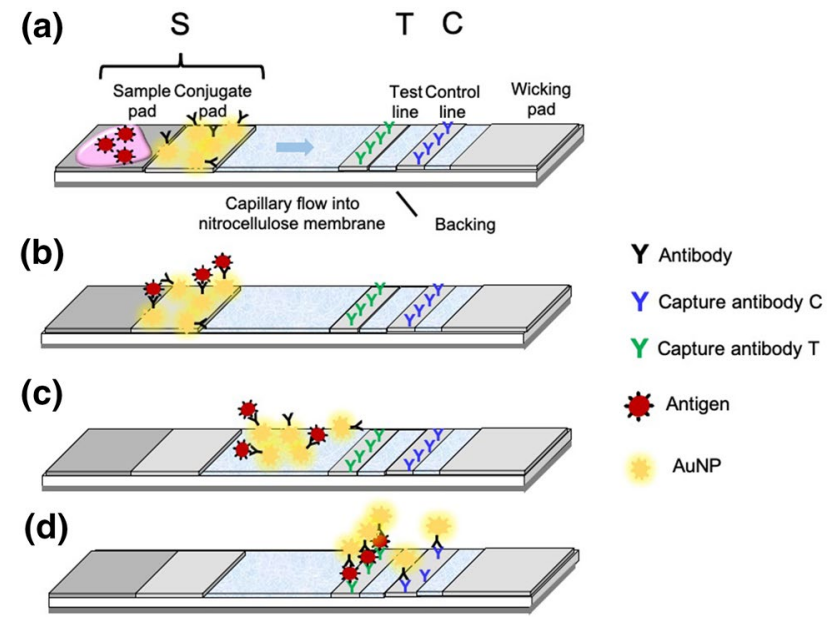

Fig. 30 Scheme showing how the LFIA in sandwich format works. a Sample is poured onto a pad placed in the window $\mathrm{S}$; $\mathbf{b}$ the antigens are bound to the NP-conjugated antibody in a second pad forming a complex antigen-antibody-NP conjugate; c the complex migrates through a capillary membrane towards the pad $\mathrm{T}$; $\mathbf{d}$ in pad $\mathrm{T}$ the complex antigen-antibody-NP conjugate links to a capture antibody forming a colored sandwich capture antibody-antigen-antibody-NP conjugate whereas the excess antibody-NP conjugates are linked to a second capture antibody in pad $\mathrm{C}$ to form a colored product. The unreacted materials are absorbed in the wicking pad

of reagents, buffer, or sample. If only a colored spot appears in the control area $\mathrm{C}$, the fluid has migrated adequately through the device, demonstrating that the device has worked correctly in the absence of target antigens in the sample, and the test is considered negative (Fig. 29c). If only the line $\mathrm{T}$ is colored the assay has failed (Fig. 29d) [85].

The competitive or inhibition format is used to detect low molecular weight molecules with a single epitope such as mycotoxins and antibiotics. In this format, the analyte competes with analyte-like proteins immobilized on the test line $\mathrm{T}$ to bind labeled antibodies. The response is negatively proportional to the analyte concentration. Therefore, the absence of color in test line $\mathrm{T}$ indicates a positive result whereas a colored line $\mathrm{T}$ denotes a negative result.

The LFIA can be performed within minutes, as a few steps are performed in small-scale, portable individual devices that accommodate crude (not purified) biofluid samples. LFIA kits use containers such as portable penside cassettes and casings. Therefore, LFIAs are suitable for in situ tests.

(c) Enzyme-linked immunosorbent assay: Developed independently and simultaneously in 1971 by the Swedish scientists Eva Engvall and Peter Perlmann for detecting immunoglobulin G [86] and by the Dutch scientists Bauke van Weeman and Anton Schuurs who made a pregnancy test [87], ELISA analyzers (cf. Fig. 27) have 
(a)

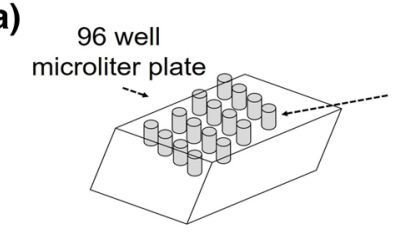

Antigen from sample

Y Primary antibody

Enzyme or label

V Enzymatic chromogen substrate

Color end product
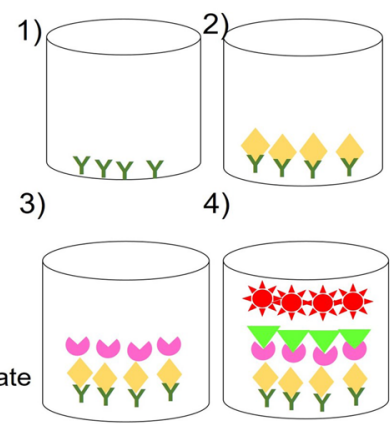

(b)
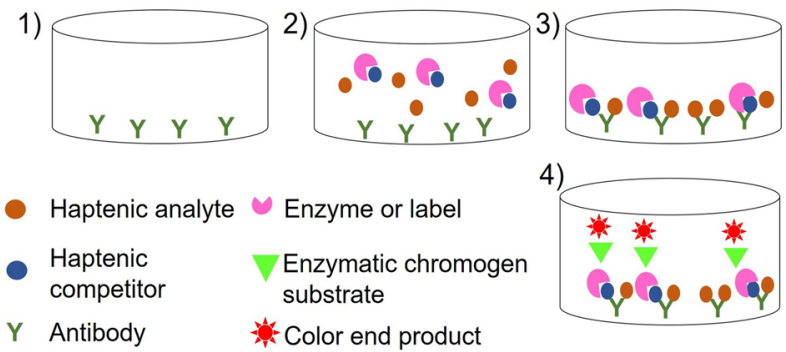

(c)

1)

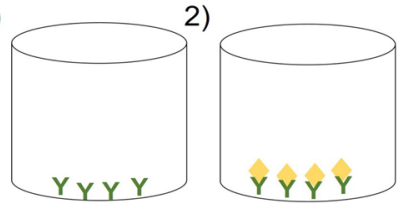

Antigen from

sample

ᄉ Primary antibody

Enzymatic chromogen
substrate

^ Secondary antibody Color end product
3)

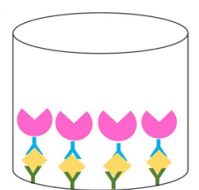

4)

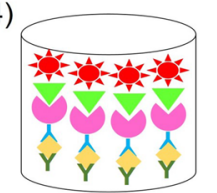

(d)

1)

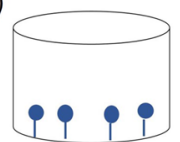

2)

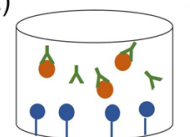

3)

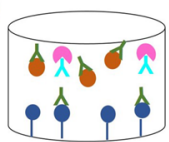

4)

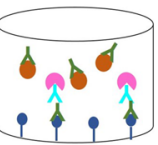

$\begin{array}{lll}\text { Haptenic analyte } & \text { Enzyme or label } \\ \text { Haptenic competitor } \checkmark & \begin{array}{l}\text { Enzymatic chromogen } \\ \text { substrate }\end{array} \\ \text { Y Primary antibody } & \text { Color end product } \\ \text { Y Secondary antibody } & \end{array}$

5)

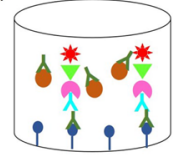

Fig. 31 Scheme of the most usual ELISA formats. a Direct binding, $\mathbf{b}$ direct competition, $\mathbf{c}$ sandwich, and $\mathbf{d}$ indirect competition or inhibition

become very popular. This technique is characterized by its higher sensitivity than that of dipstick IA and LFIA, the low amount of sample required, its easiness to be automatized, and its inexpensiveness. ELISA is a heterogeneous IA typically conducted in a well plate. The antibody or the analyte (antigen or hapten) is immobilized on the bottom of the well via covalent bonds or by adsorption and the remaining sites are blocked with sacrificial proteins. ELISA, like other types of EIAs, employs enzyme labeling. Among the enzymes most frequently used are alkaline phosphatase, glucose oxidase, and horseradish peroxidase [88]. The enzyme or label molecule catalyzes a chemical reaction that transforms many molecules of substrate into an end-product easily detectable with a colorimetric device. Alternatively, a photoluminiscent, chemiluminiscent, or an electroactive end-product can be formed [85].

Figure 31 illustrates the basic stages of the four most commonly used ELISA formats: (a) direct, (b) direct competition, (c) sandwich, and (d) indirect competition or inhibition. In the direct method the targeted analyte is attached to an enzyme-labeled antibody that is displayed by means of a suitable substrate yielding a colored end-product (Fig. 29a). In the direct competition format (Fig. 29b), primary antibodies are competitively bound to the analyte and the labeled haptenic competitors so that the larger the amount of analyte the lower the amount of label and detector signal. In the sandwich format (Fig. 29c) the primary antibody is anchored to the well bottom and then binds to the analyte (a macromolecule with at least two different epitopes). After this, the analyte is bound to a labeled second antibody forming a sandwich (Fig. 29c). In the indirect competition format (Fig. 29d), the haptenic competitor is anchored to the well bottom and then the analyte and the labeled antibody solutions are poured in. The analyte binds to the antibody and the rest of the antibodies bind to the haptenic competitors. After this, labeled secondary antibodies are bound to the haptenic competitor-primary antibody complex so that the larger the amount of analyte the lower the amount of label and signal. The two formats more frequently used are the sandwich format applied for detecting high molecular weight molecules such as foodborne pathogens and allergen proteins and the competitive format for small molecules such as antibiotics, mycotoxins, or pesticides [85, 89].

The complete ELISA process is commonly carried out in 96-well microplates made of polystyrene; however, magnetic particles, membranes with reactive strips format, microarrays of silicon derivative, plastic, etc., with flat surface format containing a high number of assay microareas are also used. An end instrument pocket photometer or spectrophotometer is required to detect the color or fluorescence of the end-products from the 
antigen-antibody labeled complexes. As a result of the multiple steps required, it is evident that ELISA requires strict protocols that include multiple operations such as freezing, thawing, vortexing, centrifuging, and pipetting samples and chemical reagents into the support, followed by multiple washing cycles to remove all unbound residues from the fluid apart from the bound analyte. As result of all these operations several hours or days are often spent completing the test [85].

\section{Microfluidics}

Classical dipsticks and their successors in immunology, dipstick IAs and LFIAs, as well as classical plate spot tests and ELISA, can be considered conventional paper-based and plate-based methods of microanalysis, respectively, because a newer generation of microanalytical methods has been developed based on microfluidics, a novel science that has flourished since the 1990s. Microfluidics is aimed at developing microanalytical devices that process amounts of sample on the microscale or less $(1 \mu \mathrm{L}$ to $1 \mathrm{~nL})$. Two microfluidic devices (MFDs) also called micro-total analytical systems ( $\mu$ TASs) are of interest here owing to their direct connection with spot tests, immunotests, and IAs: paperbased methods and some types of lab-on-a-chip (LOC) or simply "chips".

Paper-based methods: Among the MFDs, the microfluidic paper-based analytical devices ${ }^{15}$ ( $\mu$ PAD or lab-on-apaper) have become a common platform for microanalytical testing. R. H. Müller and D. L. Clegg introduced the concept of guided fluid flow inside paper for application in chromatography in 1949 [90], but it was in 2007 when George M. Whitesides et al. [91] developed the first $\mu$ PAD for the simultaneous detection of glucose and protein in urine.

Advances in $\mu$ PADs are addressed to overcome the limitations of more conventional dipsticks and LFIA diagnostic platforms. Among their advantages, $\mu$ PADs have the ubiquity and inexpensiveness of the raw material (paper and porous membranes) and the ability to handle small volumes of fluids by passive transport due to capillary forces following the Lucas-Washburn model, ${ }^{16}$ which avoids the use

\footnotetext{
15 The term "paper" in this section refers to the use of any paper or porous membrane used as support in microfluidic devices or $\mu$ PADs [85].

16 The Lucas-Washburn model describes the penetration $L$ of a liquid into a capillary pore or thin tube with time $t$ following a simplified model of diffusion.

$L=(D t)^{1 / 2}$

The diffusion coefficient $D$, characteristic of the system, is governed by both the geometry of the capillary (radius $r$ for cylindrical pores) and the properties of the penetrating fluid (dynamic viscosity, surface tension, and contact angle between the penetrating liquid and the solid pore wall) following the relationship:
}

of pumps. In addition, $\mu$ PADs have the ability to conduct assays that require sequential steps and can be designed as multiplexed setups, i.e., devices in which several assays can be conducted simultaneously (see hereafter). Since $\mu$ PADs are portable, easy to use, and instrument-free devices that do not require electrical power supply, they are a promising technology for POC and for use in settings with limited resources. In summary, $\mu$ PADs meet the affordable, sensitive, specific, user-friendly, rapid and robust, equipmentfree, and deliverable to end-users (ASSURED) criteria that the World Health Organization (WHO) has established for the diagnostics of infectious and non-communicable diseases [43, 85, 92, 93].

The $\mu$ PADs are small pieces of paper or porous membranes in which is made a pattern of channels. The design and manufacture of $\mu$ PADs are firstly determined by the method of patterning of the support. Broadly speaking, patterning methods can be classified into physical, by cutting the paper, and chemical patterning that includes modification or functionalization of the fibers by blocking the pores using photoresist agents or specific polymers (polydimethlysiloxane, or photoreactive acrylate and methacrylate) as well as printing, plotting, masking, or stamping the pattern, which previously was drawn and drafted with a computer program [93]. The micropatterning technique of plotting barriers on the paper support with hydrophobic ink loaded into a pen has become a standard method. Hydrophobic wax- or polystyrene-based inks are used for delimitating the hydrophilic channels. Dimensions of the channels and barriers are down to 400 and $200 \mu \mathrm{m}$, respectively (Fig. 32) [43, 92]. Dipsticks combining the NP technologies with inkjet-print technique, already described previously $[67,68]$, are a good example of the inkjet printing technique (cf. Fig. 23). Printing can also be done using laser, flexographic, and screen techniques. Masking techniques include photolithography, plasma etching, and chemical vapor deposition. Stamping is done with a patterned stamp with waxes or polymers previously heated

Footnote 16 (continued)

$L=\sqrt{\frac{\gamma r t \cos (\varnothing)}{2 \eta}}$

The Washburn equation developed by Edward Wight Washburn in 1921 (Washburn EW (1921) The Dynamics of Capillary Flow. Phys Rev 17: 273) is also called Lucas-Washburn equation as Richard Lucas proposed a similar model 3 years earlier (Lucas R (1918) Ueber das Zeitgesetz des Kapillaren Aufstiegs von Flussigkeiten. Kolloid Z. 23: 15). Sometimes this equation is called the Bell-Cameron-Lucas-Washburn equation, considering that J.M. Bell and F.K. Cameron's developed the form of the equation in 1906 (Bell JM, Cameron FK (1906) The flow of liquids through capillary spaces. J Phys Chem 10: 658-674). 
(a)

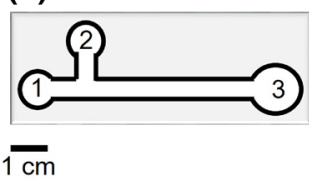

$\square$ Hydrophylic channel

- Hydrophobic barrier

1 Sample zone

2 Reagent zone

3 Test zone (b)

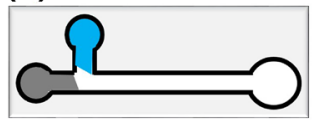

(c)

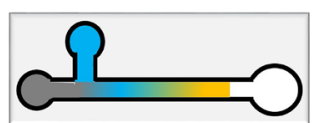

(d)

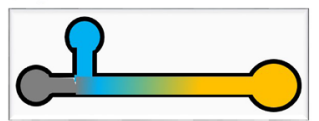

Fig. 32 Scheme of a simple wax-based ink printed $\mu$ PAD. a Description of the different zones of the device; $\mathbf{b}$ initial stage of the assay with sample and reagent confined in their zones; $\mathbf{c}$ sample and reagent flow through the hydrophilic channel and start to react; $\mathbf{d}$ end of the assay with the end-product of the reaction reaching the test zone. Adapted from [92]

that harden by cooling or using inks that are stamped and dried at room temperature [93].

The adaptation of the $\mu$ PADs to the intended application requires a customization that encloses operations such as lamination of the channels with a plastic film, stacking of several patterned layers of paper to obtain a three dimensional (3D) device, incorporation of detectors or electrodes, addition of chemicals for controlling wicking, or addition and drying of reagents in the $\mu \mathrm{PAD}[93,94]$.

$3 \mathrm{D} \mu \mathrm{PADs}$ are designed as $2 \mathrm{D}$ patterns of channels in two or more paper sheets stacked alternating with hydrophobic sheets (a double-side tape or a polymer sheet fixed with adhesive spray) in which are located holes filled with hydrophobic powdered material that vertically connect the two 2D horizontal channel networks in the above and underneath paper sheets. Thus, the fluids can move in all three dimensions $(x, y, z)$. The channel patterns and holes can be made using any of the described methods above, e.g., photolithographic method by exposing the paper sheet to UV light with a previously fixed negative photoresist and a photomask that cross-links the photoresist in the designed channels pattern (Fig. 33). The main advantage of 3D $\mu$ PADs is that they enable to one conduct simultaneously multiple tests by distributing a sample into distinct channeled circuits in the $x y$ plane of the support and in the orthogonal $z$ direction [93-95]. By overlapping multiple layers it is possible to increase the number of tests and samples that can be conducted simultaneously (e.g., two separate tests on two separate samples, thus performing multiplex tests).

Detection in $\mu$ PADs in the majority of devices does not require an attached readout instrument because in most cases the detection strategy is based on a color change observed with the naked eye. Semiquantitative colorimetric determination is made by comparison with calibration color charts, measurement of the distance along the channel where the color change occurs, or chronometric detection based on the time that the fluid takes to wick across the channel. Readout instrumentation based on DIC is required for quantitative
Fig. 33 Scheme of a 3D $\mu$ PAD for conducting the independent analyses of two separate samples. a Front view of the patterned paper layer. From left to right is shown the evolution of a test performed with two samples that flow simultaneously through the 3D channel network towards the reagent spot. b Cross-section view of the paper sheet section in dotted lines in a. c Oblique view showing the three layered $\mu$ PAD structure that includes a top patterned paper layer, an intermediate hydrophobic layer patterned with hydrophilic vertical channels, and a bottom patterned paper layer. Adapted from [94] (a)
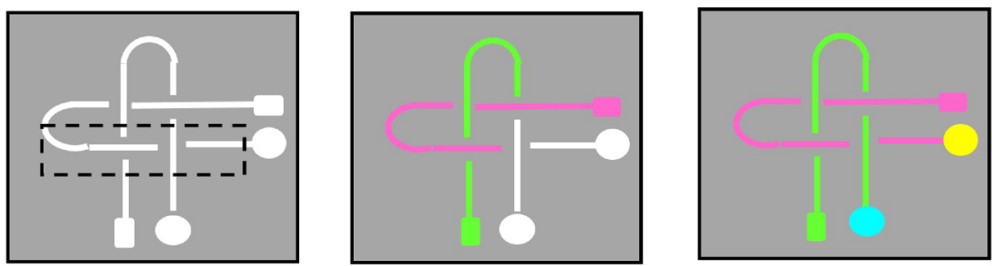

(b)
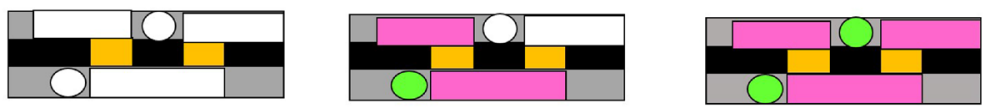

(c)

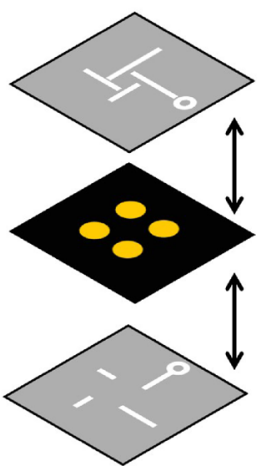

Top layer of patterned paper

Tape with

patterned sealed holes

Bottom layer of patterned paper

\section{Photoresist}

\section{Paper}

Hole sealed with cellulose powder

- Tape

$\square$ Fluid 1

Fluid 2

Endproduct 1

Endproduct 2 
Fig. 34 Scheme of the basic structure of an ePAD: a simplest ePAD with an electrochemical test zone; $\mathbf{b}$ ePAD with color and electrochemical test zone; and $\mathbf{c}$ multiplexed ePAD for performing three simultaneous electrochemical assays from a single sample. Adapted from $[97,98]$ (a)

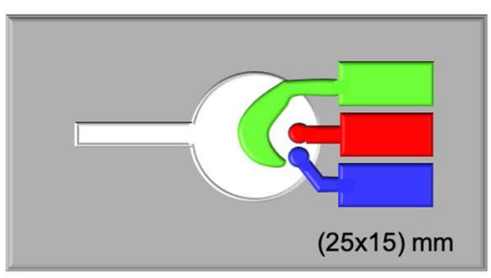

(b)

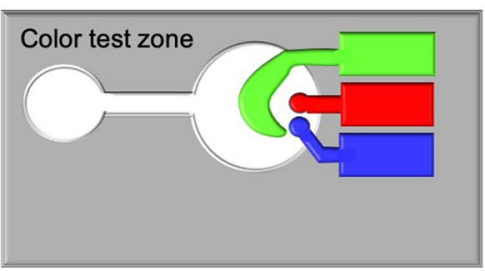

(c)

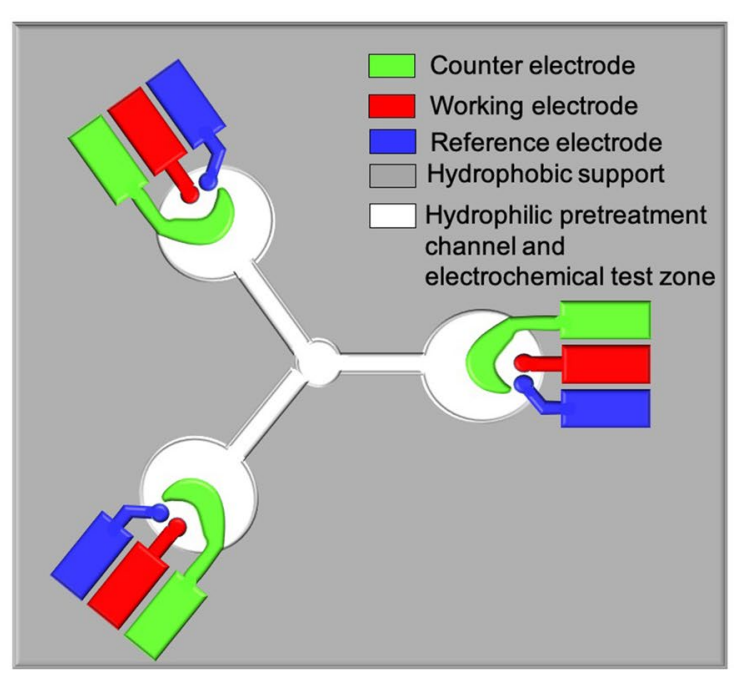

colorimetric assays. CL, chemiresistive,${ }^{17} \mathrm{ECL}$, and electrochemical detectors are also used owing to their reduced dimensions. Paper-based microfluidic ECL devices have three layers: a top transparent plastic layer, a middle layer where the reaction takes place, and the bottom layer with SPEs $[70,74]$. The compact $\mu$ PADs with integrated electrochemical sensors are easy-to-use sensor strips called electrochemical PADs (ePADs) and work with samples of ca. $50 \mu \mathrm{L}$. The fabrication of an ePAD has the added difficulty of integrating the electrode system in the microfluidic platform of the $\mu \mathrm{PAD}$ and thus inkjet, screen, or stencil printing and microwire placement on flexible or paper-based substrates have been developed as more suitable alternative methods to the standard manufacturing of semiconductorbased electrochemical sensors. The electrodes are manually patterned onto the paper surface or ink-printed, stencil printed, or screened (screen-printed electrodes) SPEs [93]. Carbon, graphite doped with electrode modifiers, noble metals, and NPs are used as patterning materials. $\mathrm{Ag} / \mathrm{AgCl}$ ink is commonly used for making the reference electrode [93, 96]. Figure 34 shows the scheme of the most common structures of ePAD, irrespective of the method of manufacturing used. Figure 34a shows the simplest structure of an ePAD with a hydrophilic channel or pretreatment zone where the sample and reagents are dropped and flow until reaching

\footnotetext{
17 Chemiresistor is a chemical sensor made of a sensing material (metal oxide semiconductors, conductive polymers, or nanomaterials such as GR or CNTs) whose electrical resistance changes with the nearby analyte. The analyte can establish different bonds (covalent, hydrogen bond, molecular recognition, etc.) that modify the electrical behavior of the sensor. Basically, the device is integrated with two electrodes between which is interposed the sensing material. The latter, in contact with the analyte, modifies its electrical resistance that is measured by a reader located in the external circuit that connects both electrodes.
}

the electrochemical test zone containing a three-electrode device. All these components are patterned on a hydrophobic substrate. Figure $34 \mathrm{~b}$ shows a similar ePAD structure that includes a color and electrochemical test zone that enable two complementary detections. Figure $34 \mathrm{c}$ depicts a more sophisticated multiplexed ePAD for performing three simultaneous electrochemical assays from a single sample that is dropped in the central pretreatment zone and flows through the channels to the three separate electrochemical test zones [97, 98].

ELISAs have also been added into the list of testing methods that have been miniaturized as microfluidic paper-based devices. The paper-ELISA (P-ELISA) device has been made by wax printing in a paper sheet 96-microzone plates that mimic the 96-well plate of the conventional ELISA. The successive steps of ELISA are conducted in each microzone like in the conventional method. The colorimetric detection is carried out by DIC. A 3D microfluidic device replaces pipetting steps with sliding operations. This method enables POC testing [85].

Lab-on-a-chip devices: The first LOC was developed in 1975 by the US scientist S. C. Terry as a miniaturized gas chromatograph. Since then, this field has notably grown [99]. A basic LOC is a microchip in which basic operation units are integrated for performing a complete analytical process: preparation, extraction, reaction, and detection. The channels, pumps, valves, filters, sensors, detectors, and other pieces are made on a miniaturized substrate of silicon, metal, polymer, glass, paper, or porous membranes among other materials. Five methods for liquid propulsion are used in microfluidic platforms: capillary, pressuredriven, centrifugal, electrokinetic, and acoustic. Detection techniques include a wide range of technologies from the simplest change of color observed with the naked eye and semiquantitative determination by comparison with 
Fig. 35 a Scheme of a miniaturized LOC device. Top and perspective view. b Scheme of a LOC for POC diagnostic. Adapted from [101]

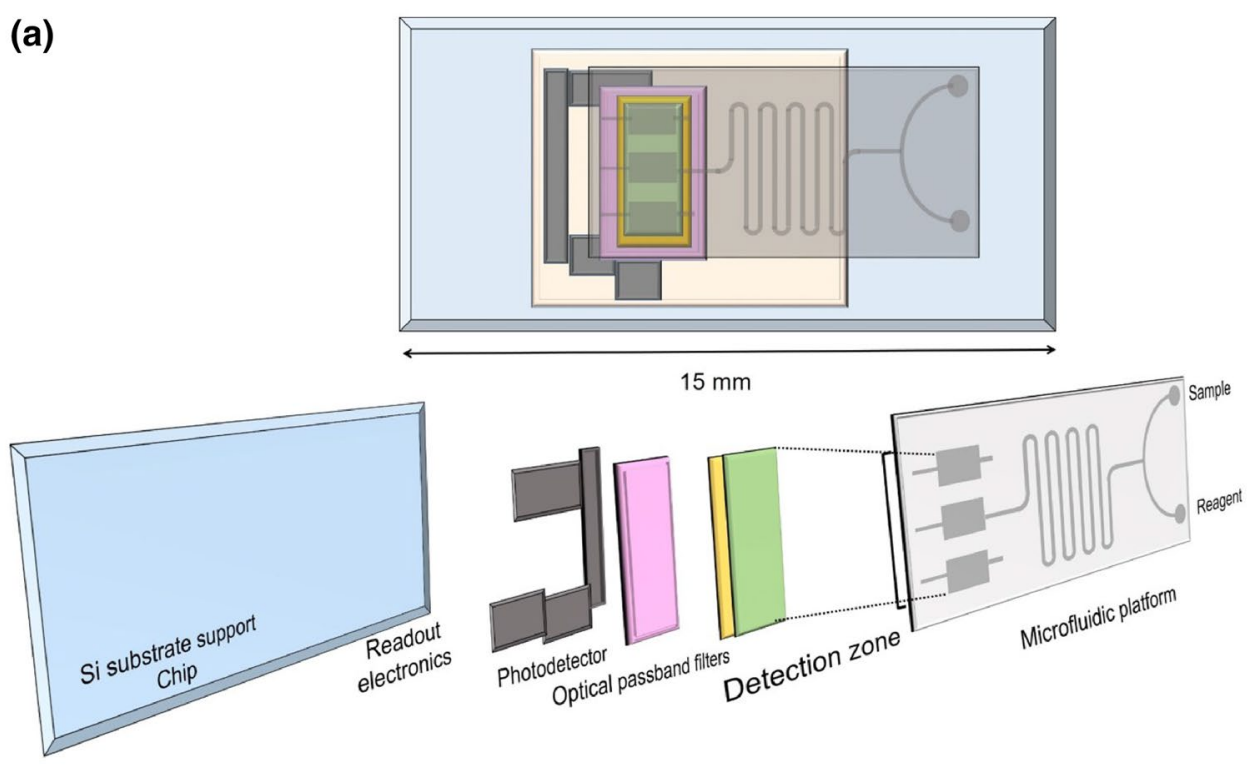

(b)

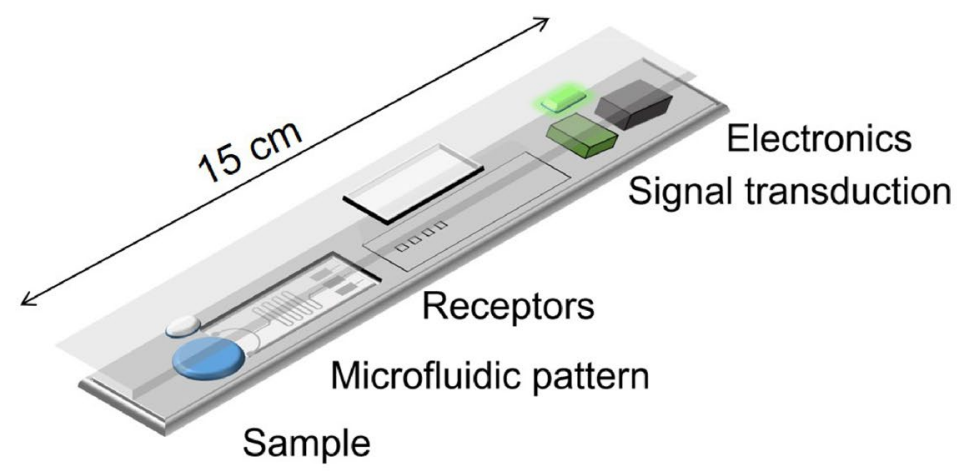

calibration charts to DIC, UV-Vis spectrophotometry, electrochemical devices, mass spectrometry, chemiluminescence, and fluorescence analysis [100]. As for $\mu$ PADs, the main advantages of LOCs are their ability to perform several tests in parallel on the same sample. A variety of laboratory prototypes and commercial devices have been developed, which have wide applications in microanalysis. Two analytical LOC devices are shown in Fig. 35a and b as examples illustrating how these devices work. In the miniaturized device shown in Fig. 35a the different components are overlapped in successive layers on a silicon substrate chip. On the top is placed the microfluidic platform with microcapillary channels (approximately $15 \mathrm{~mm}$ length, $10 \mu \mathrm{m}$ depth, and $50 \mu \mathrm{m}$ width) that end in the detection zone. Underneath the detection zone are placed a series of optical passband filters and the photodetector. Around that are located the readout electronic circuits. In the POC device a more compact system is integranted in a portable cartridge (Fig. 35b) [101].

A third example is the portable electrochemical LOC controlled by a smartphone platform shown in Fig. 36. The device is integrated with an ePAD or other microfluidic device with miniaturized or microscale three-electrode sensor used along with a customized electronic board containing an audio jack unit and other electronic elements that are connected to a smartphone. The use of miniaturized electrodes enables working with a small time constant [72], fast mass transport, and low ohmic and capacitive current. A customized app processes the data from the audio jack system of the phone, used as sensing interface, and draws the spectrum with the built-in digital camera. The audio jack system can generate smooth waveforms by controlling the tone frequency which enables one to conduct the voltammetric assays. Contributions by Scholz as well as by Mirceski et al. on fundamentals of voltammetric techniques can be found in ChemTexts [102, 103]. Alternatively, the audio jack also enables the transference of control signals between the phone and an external potentiostat [75].

Hybrid microfluidic devices or hybrid chips have been developed for POC testing by integrating passive fluid flow PADs with active microfluidic chips that provide better performance in conducting and transporting liquid. A 

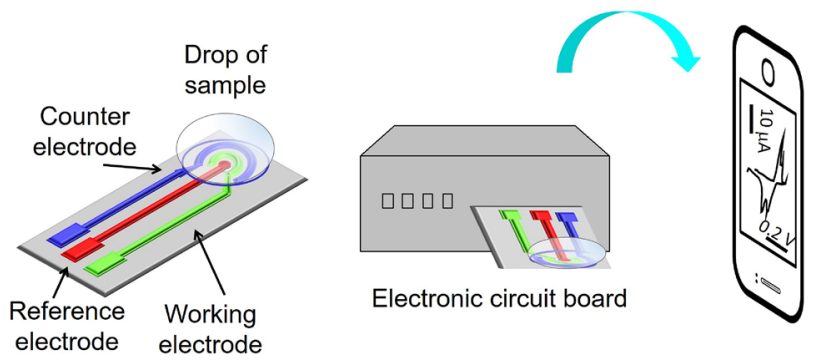

Fig. 36 Scheme of a three-electrode sensor. The assay is performed on the working electrode of the electrochemical sensor that is inserted into the board circuit containing the audio jack and other electronic components. An app in the smartphone controls the development of the electrochemical test and the processing of raw data. Adapted from [75]

miniaturized paper/poly(methylmethacrylate) (PMMA) hybrid microfluidic ELISA is a good example of this kind of appliance [104].

\section{Multiplex spot test and multiplexed immunotests}

Multiplex or multiplexed tests are systems (arrays) that enable the simultaneous detection of multiple analytes (multiparametric measurements) from a single sample under standard conditions. For this reason, these analytical methodologies address a wide range of applications, especially in the fields of clinical diagnostic, food, and environmental sciences. Among the advantages of multiplex tests are the requirement of a small amount of sample, ca. $25-50 \mu \mathrm{L}$ compared with $100 \mu \mathrm{L}$ per target required for ELISA. In addition, multiplex tests maintain linearity over three-five orders of magnitude more than ELISA, which is essential when the sample contains analytes that are present at different concentrations. A variety of designs have been proposed for performing multiplex tests, and microfluidic technologies have played an essential role [105]. Similarly, the previously described methods for performing IAs have been the basis for developing multiplex immunoassays (MIAs) [106].

Multiplex tests can be divided into two general categories $[63,106]$ :

- Site-encoded identification (planar microarrays). The detection of multiple analytes is performed by immobilization on a solid surface with spatially separated test zones. It can be used with a unique or multiple probes for detecting analytes with or without labels. Examples of multiplex tests with monoprobes are ELISA plates and LFIA conducted in monolabel mode through a linear membrane. The labeled antibody-analyte-capture antibody for each analyte is immobilized in separate test lines (LFIA) (Fig. 37) or in microtiter plates or wells (ELISA). Other site-encoded platforms are multiplexed microarrays that consist of a solid

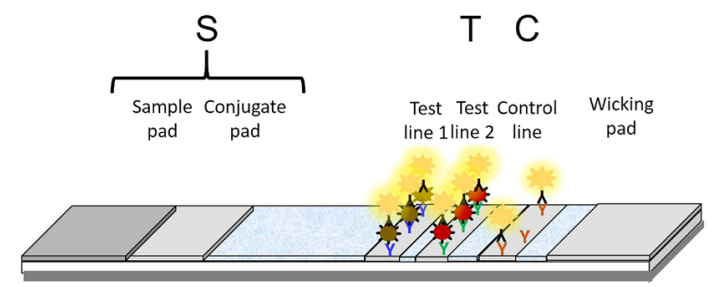

Fig. 37 LFIA with two test lines that enable the identification of two different antigens (brown and red) present in the same sample using the same antibody-AuNP conjugate (black-yellow) label and two capture antibodies (blue and green) specific for each antigen present in the sample

planar surface such as glass slides or silicone films where reagents are immobilized in a set of well-organized miniaturized reaction areas. Here, a colorimetric or luminescent system is used for detection. Array-based biosensors are also included in this category of multiplexed tests.

The multiprobe mode includes a different detector for each analyte (i.e., chemical reagent, enzymes, fluorescence dyes, NPs, or electrochemiluminescent, radioisotope probes, etc.). A simple multiplex spot test in multiprobe mode can be conducted in branch-shaped paper devices designed with a multiarmed (i.e., five-leaved) configuration, so that separate reagents, specific for each analyte, are placed in the end of each branch (Fig. 38). A drop of sample is poured into the bottom of the branched paper device. The drop flows by capillary forces until reaching each reagent at the different reaction points enabling colorimetric identification [107].

Another simple 3D multiplex $\mu \mathrm{PAD}$ is prepared by folding three types of paper sheets with sample windows, channeled patterns, and reagents deposited in spots, respectively, using photolithography according to the principles of origami [108]. Figure 39a shows the fivesheet $\mu \mathrm{PAD}$ that enables the simultaneous analysis of four samples with eight reagents. Figure $39 \mathrm{~b}$ illustrates the folding procedure for the first series of four analyses and Fig. 39c illustrates the folding procedure for the second series of four analyses.

Detection with multiple labels (multiprobe) can also be performed in MIAs. One of the most simple MIAs is the multiplex LFIA that enables the identification of several analytes with several labels. The "traffic light" test is an example of a multilabel LFIA method. The principle of the traffic light test is the competition for binding the QD-labeled antibodies between the analyte in the sample and the hapten-protein immobilized on the surface of the test lines. Thus, the greater the concentration of analyte in the sample the lower the color intensity in its test line. Figure 40a shows a two-analytes traffic light LFIA before the analysis with the specific labeled antibodies in the conjugate pad and the competi- 

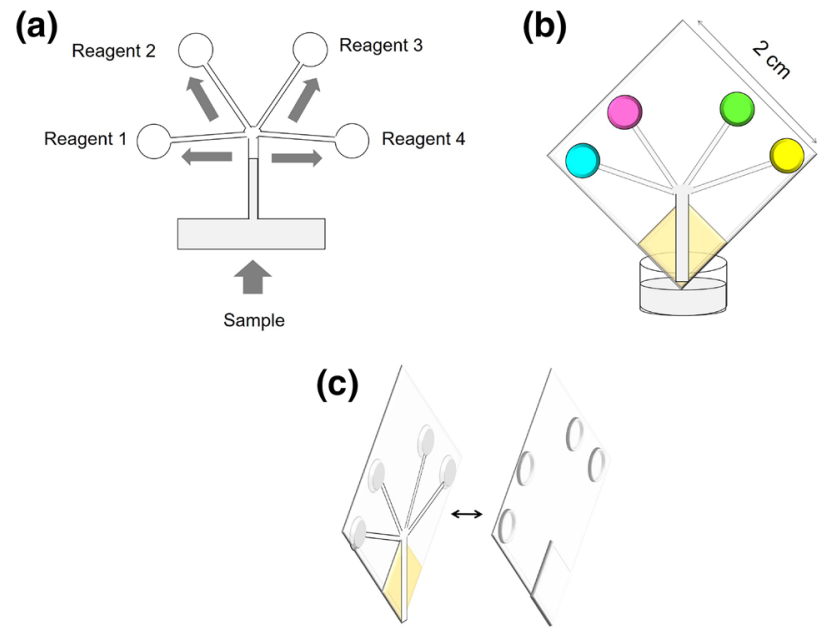

Fig. 38 a A drop of the fluid sample is poured into the bottom of the branched strip and spontaneously flows by capillary forces throughout the trunk and the four branches. b When the sample reaches the different reagent places, a color reaction occurs that enables the identification of each one of the analytes present in the sample. c Perspective view showing the two layers of the $\mu \mathrm{PAD}$, the bottom sheet with the patterned channels and reagent places with the dried reagent spots (left) and the protecting top layer with reagents and sample windows (right). Adapted from [107]

tor hapten-proteins in the test lines. In Fig. 40b a drop of the sample, composed only of analyte 2 , is placed in the sample pad. Identification of analyte 2 is observed by the red color of the complex hapten-labeled antibody retained in the test line 1 and the absence of color in line 2 because no labeled antibody has been retained in this test line. In Fig. 40c a drop of the sample, composed only of analyte 1 , is placed in the sample pad. Identification of analyte 1 is observed by the yellow color of the complex hapten-labeled antibody retained in test line 2 and the absence of color in line 1 [109].

Another type of multiprobe multiplex assay called flow-through tests is performed in microcolumns in which the sample flows along several column layers that contain specific reagents or antibodies to the analytes [106].

An example of site-encoded multiplexed LOC is the SlipChip test shown in Fig. 41. Several reagent solutions are loaded and allowed to dry in a portable plate with a row formed by individual microwells. The sample is loaded in a second row of microwells. The sample wells can be individually connected to the reagent wells by slipping the chip cover that has a channeled design that enables the flow of the sample solution towards the adjacent well where the analyte reacts with the specific reagent (Fig. 41a). Conversely, the SlipChip device can be prepared for analyzing several samples with a single (a)

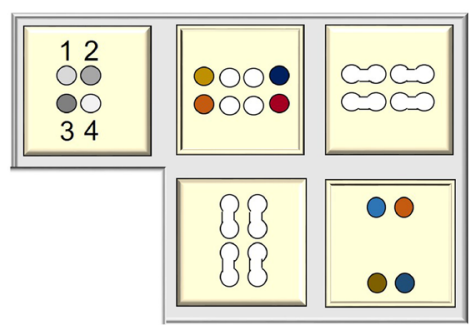

(b)
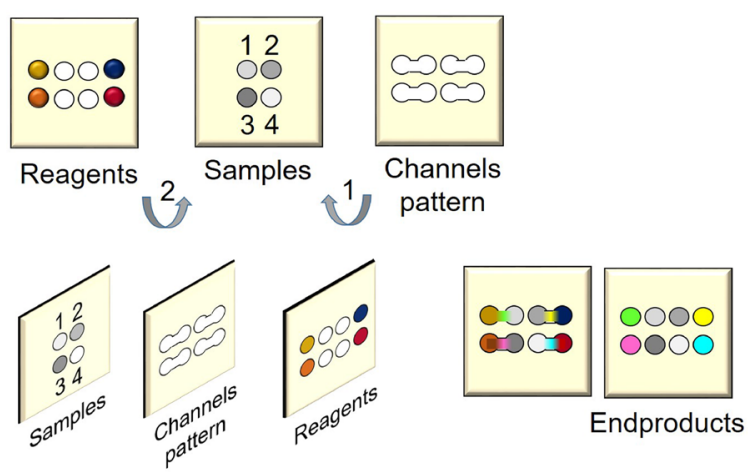

(c)
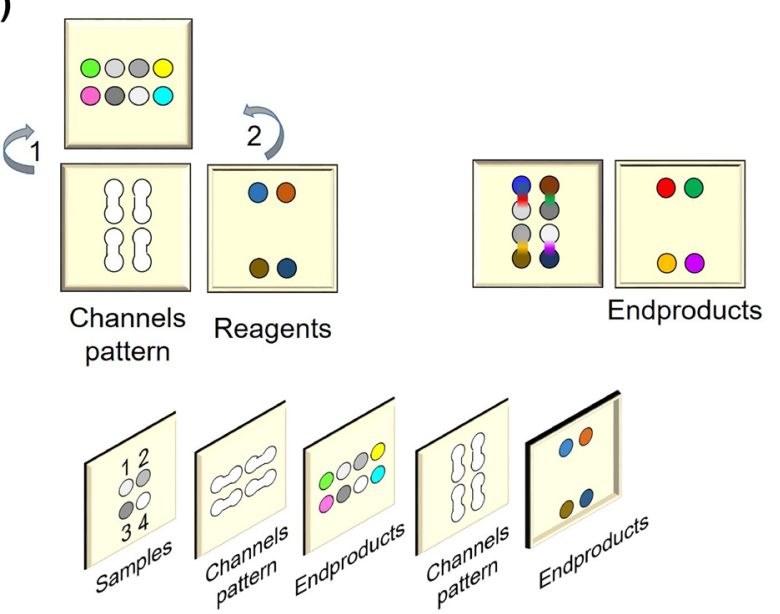

Fig. 39 a Multiplexed 3D $\mu$ PAD of origami type. a Scheme of the five panels used for performing the analyses. b Sheet folding procedure for the first series of four analyses. $\mathbf{c}$ Sheet folding procedure for the second series of four analyses. Adapted from [108]

reagent (Fig. 41b) or for the simultaneous analysis of several analytes in several samples (Fig. 41c) [110].

- Label-encoded multiplexed platforms or multiple quantitation tags (planar microarrays and non-planar or suspension arrays). In this type of multiplex test the discrimination of analytes is due to the attachment to multiple labels that are encoded carriers differentiated by their intrinsic properties. Beads and microspheres in which are trapped dyes with a specific emission spectrum that act as a unique code are typical encoding carriers. Other optical encoding carriers are QDs and upconversion NPs. Structural surface color-encoding carriers are based on the interference of light generated through photonic crystals that have a modu- 
Fig. 40 a LFIA before analyses with two analytes in the sample drop, the specific labeled antibodies in the conjugate pad, and the hapten-protein conjugates in the test lines; b LFIA with a positive result for analyte 2 via colorless test line 2. c LFIA with a positive result for analyte 1 via colorless test line 1 . Adapted from [109]

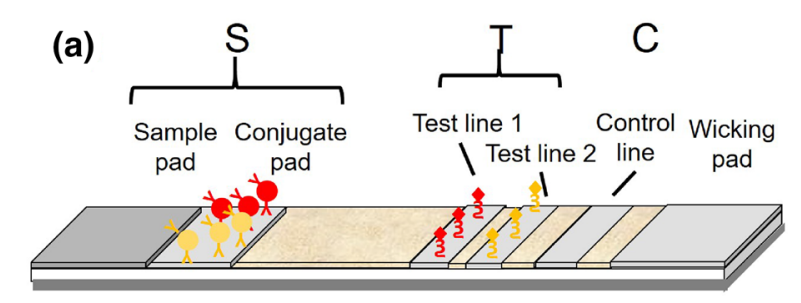

(b)

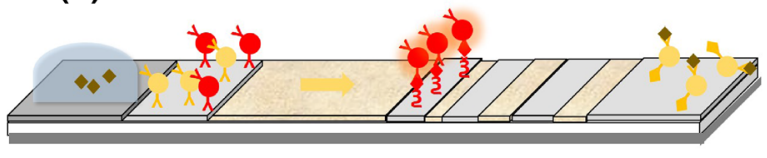

Hapten 1-protein conjugate

Hapten 2-protein conjugate

Antibody 1-QD 1 conjugate

Antibody 2-QD 2 conjugate

- Analyte 1

- Analyte 2

(c)

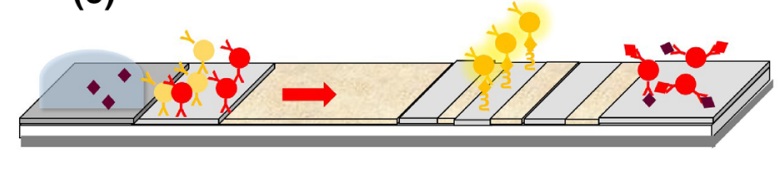

(a)

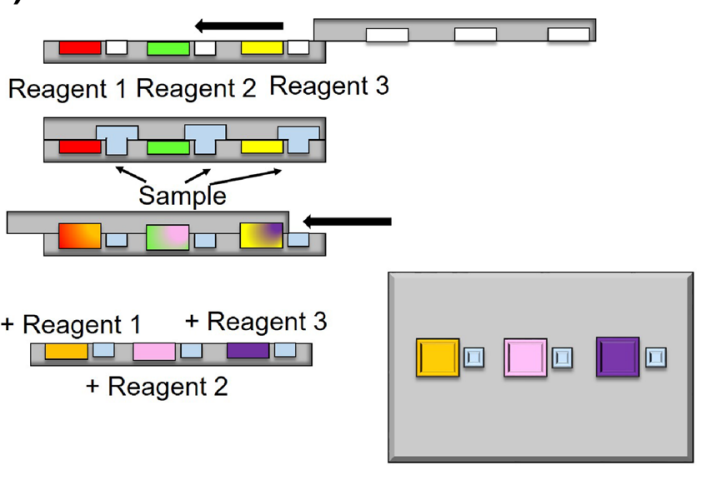

(b)

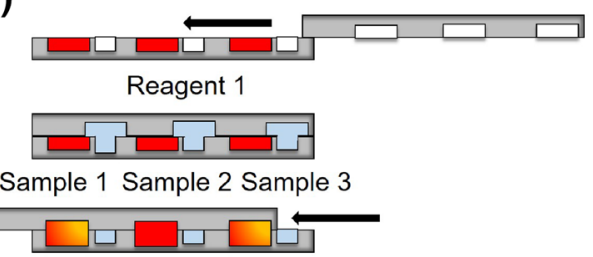

+ Sample 1 + Sample 3

- Sample 2

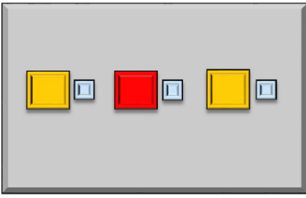

(c)

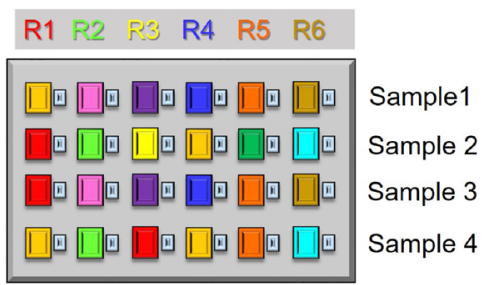

Fig. 41 Scheme of the SlipChip devices: $\mathbf{a}$ analysis of a single sample with three reagents; $\mathbf{b}$ analysis of three samples with a single reagent; $\mathbf{c}$ mosaic of multiplex SlipChip test performed with six reagents located in each column and four samples located in each row. Adapted from [110]

lated dielectric constant and a photonic bandgap. SERS barcode particles are composed of a plasmonic nucleus for the generation of the electric field, a Raman reporter for the unique vibrational fingerprint, and a coating layer that enables chemical functionalization and prevents code leaching. Electrochemical tags are specific immunoreagent labels. Redox-active molecules such as dyes (thionine, toluidine blue, or methylene blue) and heavy metal ions $\left(\mathrm{Cu}^{2+}, \mathrm{Cd}^{2+}\right.$, or $\left.\mathrm{Pb}^{2+}\right)$ are redox species potentiometrically detected. ${ }^{18}$ Shape encoding uses different shapes to generate the codes. Silica NT with different hollow structures and silica-polymer coated particles fabricated with optoflu-

\footnotetext{
18 Potentiometric devices effectively measure the potential difference between the working electrode and a reference electrode. Detection can be made either voltammetrically (measuring the current upon application of time-dependent potential inputs) or amperometrically (measuring the current upon application of a constant potential input or a potential pulse).
} 
Fig. 42 a LFIA before analyses with two analytes in the sample pad, specific QD-labeled antibody 1 and 2 in conjugate pad, capture antibody 1 and 2 in test line and capture antibody in control line. b Analytes 1 and 2 bind conjugated antibody-QDs 1 and 2 , respectively, in the conjugate pad and migrate. $\mathbf{c}$ Conjugated antibody-QD-analyte-capture antibody 1 and 2 complexes are formed in the test line. The unbound conjugated antibody-QDs are captured in control line by the capture antibody. Adapted from [112]

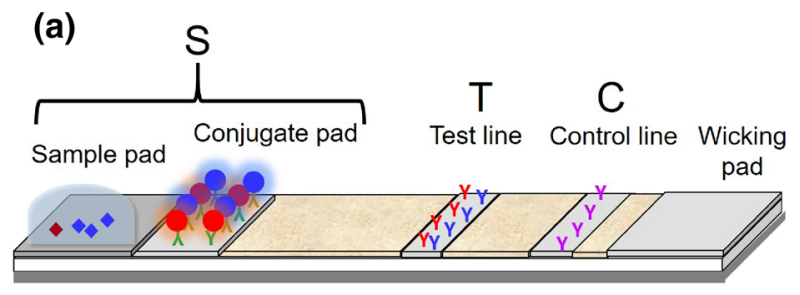

(b)

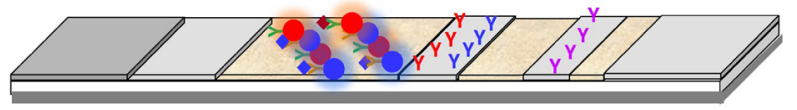

(c)

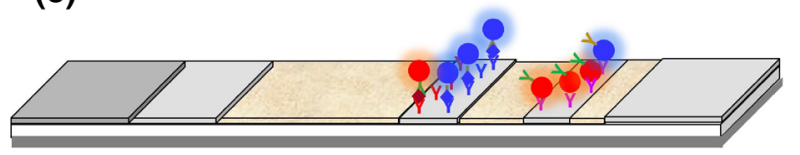

- Analyte 1

- Analyte 2

Antibody1-QD 1 conjugate

Antibody 2-QD 2 conjugate

y Capture antibody 1

Y Capture antibody 2

Y Capture antibody control idic maskless lithography are common encoded carriers of this type. Graphical encoding generates a pattern of holes or optical elements on a microcarrier such as striped rods, ridged particles, and dot-patterned particles. Lithographic methods also allow the fabrication of graphically encoded particles with novel encoding patterns. Magnetic encoding uses magnetic properties of some NPs such as $\mathrm{Fe}_{3} \mathrm{O}_{4}$, $\mathrm{Fe}_{2} \mathrm{O}_{3}$, and $\mathrm{CrO}_{2}$. Multisegmented metallic nanowires combine magnetic and optical coding. Hybrid encoding methods combine fluorescent encoding with organic dyes or QDs with shaped, structural color, SERS, or magnetic encoding. Other encoding carriers are chemically biofunctionalized beads with labels such as enzymes, metal ions, redox tags, and QDs. For example, QD barcoded microbeads functionalized with reagents or droplets prepared from solutions barcoded with known nucleic acid sequences or fluorophore combinations or concentrations are used to form suspension arrays [63, 105, 111].

Nevertheless, label-encoded multiplexed planar microarrays can be as simple as an LFIA device (Fig. 42). A simple method of this category is the multiplexed multicolor NPs LFIA based on the detection of multiple analytes by immobilization in a single test zone. Before the analysis the sample, containing two analytes, is placed in the sample pad. Specific antibodies labeled with two different QDs are immobilized in the conjugate pad. A couple of specific capture antibodies 1 and 2 are placed in the test line pad and a third series of capture antibodies is fixed in the control line (Fig. 42a). Analytes 1 and 2 are attached to the QD-labeled antibody conjugates 1 and 2 and form analyte-QD-labeled antibody complexes in the conjugate pad (Fig. 42b). The analyte-QD-labeled antibody complexes 1 and 2 are anchored by capture antibodies 1 and 2 in the test line and the unbound QD- labeled antibody conjugates are captured by the capture antibody in the control line (Fig. 42c). The positive result for analytes 1 and 2 is observed simultaneously in the test line as the QD-labeled complexes emit light at different wavelength depending on the QD-labeled antibody conjugate-analyte-capture antibody complex formed [112].

Most label-encoded multiplexed platforms are nonplanar arrays. The most common non-planar array is the flow cytometer that is a microfluidic instrument that measures a specific property (i.e., fluorescence) of the analyte immobilized in fluorescent marked beads or particles in suspension. The suspension flows through a fine capillary column where the analytes are sequentially detected with a suitable readout device (i.e., a laser light source that irradiates the suspension coupled with a spectrophotometric sensor) (Fig. 43) [113].

Development of more sophisticated microfluidic systems has promoted the growth of platforms for multiplexed assays owing to the capacity for suitable fluid routing and handling in small size devices. A number of technologies have been incorporated into the design of multiplex testing devices in addition to the already described surface-patterned platforms and microbeadbased tests: $\mu$ PAD formats (i.e., a 96-well ELISA on photoresist patterned paper or barrier-free $\mu$ PAD made by stacking two paper membranes with different wicking rates); compact disck (CD) formats for centrifugally driven fluid actuation; electrowetting-based digital microfluidics platforms that improve the sample handling; homogeneous and heterogeneous tests based on hydrogels used with electrophoresis support; large-scale integration of microreactors for heterogeneous assays; label-free detection (i.e., conjugated aptamer redox tags 


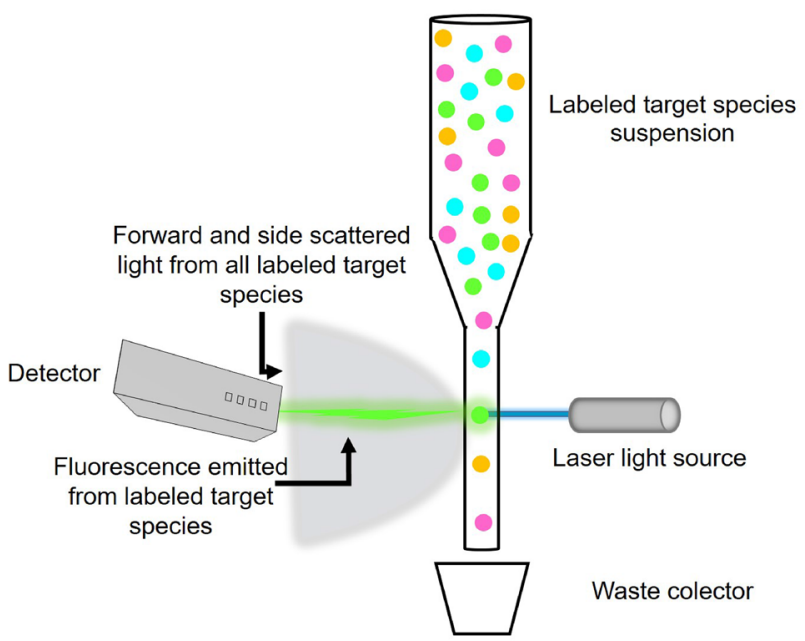

Fig. 43 Scheme of a flow cytometer

and electrochemical sensors) and hand-powered multiplexed platforms for POC applications [105].

LOC devices for massive parallel analysis have already been developed that can process simultaneously up to millions of assays. Examples of these appliances are the microarrays, bead-based assays, and pyro-sequencing platforms of picowell-plates used for gene expression and sequencing [114].

Multiplexed testing for on-site food testing, water and air quality screening, or emerging applications such as personalized monitoring, global healthcare, battlefield diagnostics and space research are driving the future of multiplexed testing towards the development of largescale platforms and digitally connected networks of portable devices [115].

\section{Forensic sciences}

Application of spot tests in forensic sciences dates back to the eighteenth century with the German-Swedish chemist Carl Wihelm Scheele (1742-1786), who in 1773 developed a spot test for detecting arsenious oxide in corpses. Further, the German pharmacist and chemist Valentin Rose (the younger) (1762-1807) in 1803 expanded this work by applying it to the identification of poisons in the walls of the victim's stomach $[116,117]$. The British chemist James Marsh (1794-1846) introduced in 1832 toxicology as an experimental science in forensic practice, which studies the effect of drugs and poisons on the human body. This scientist is known for the Marsh test that enables the identification of arsenic in a sample of human fluids using sulfuric acid and arsenic-free zinc as reagents. The reaction results in releasing arsine $\left(\mathrm{AsH}_{3}\right)$ gas that, after ignition and condensation of the formed arsenic on a cool surface, is recognized by the silvery-black deposit of metallic arsenic [118]. The first assay in serology was conducted by the Spanish born French scientist Mathieu-Joseph-Bonaventure Orfila (1787-1853) in 1827. This scientist developed a test for blood based on the solubility of the components of a bloodstain in water, and the behavior of the aqueous extract toward a number of reagents such as litmus paper [119].

Nowadays, forensic sciences encompass a large number of branches among which one can find several that use spot tests. In addition to the aforementioned toxicology and serology, detection and identification of illicit drugs and other hazardous materials such as explosives have to be mentioned [120].

\section{Serology}

There are three fluids of principal interest: blood, semen, and saliva. Other body fluids of interest are vaginal fluid, urine, and sweat.

Blood: Presumptive tests for blood detection are classed into catalytic tests (i) and chemiluminiscence and fluorescence tests (ii). Catalytic tests are based on the peroxidase activity exhibited by hemoglobin and a number of its derivatives. The guaiacum test also known as van Deen's test or Day's test was the first catalytic test devised for forensic purposes. This test is based on the work of the German-Swiss chemist Christian Friedrich Schönbein (1799-1868) in 1863 that was extended by the Dutch physiologist Izaak A. van Deen (1804-1869) on the guaiacum reaction with blood. This test uses the reaction of guaiaconic acid $\left(\mathrm{C}_{20} \mathrm{H}_{24} \mathrm{O}_{5}\right)$, the main component of the guaiacum resin exuded by Guaiacum officinale and Guaiacum sanctum trees that grow in South America and the West Indies. Guaiaconic acid is oxidized to guaiacum blue $\left(\mathrm{C}_{20} \mathrm{H}_{20} \mathrm{O}_{6}\right)$ in the presence of Fe-containing blood. The benzidine or Adler's test, developed in 1904 by the Austrian physician Oscar Adler (1879-1936) in collaboration with Rudolf Adler, is based on the oxidation of benzidine ( $p$-diaminodiphenyl) to benzidine blue with peroxide catalyzed by blood. Since the 1970s this test is seldom used because of the carcinogenic character of benzidine [121, 122]. Currently used is the Kastle-Meyer or phenolphthalin test developed by the American chemist Joseph Hoeing Kastle (1864-1916) in 1901 and that was further improved by the German physician and chemist Erich Meyer (1874-1927) in 1903. This test is based on the peroxidase catalytic activity of hemoglobin for oxidizing phenolphthalin $\left(\mathrm{C}_{20} \mathrm{H}_{16} \mathrm{O}_{4}\right)$ to phenolphthalein $\left(\mathrm{C}_{20} \mathrm{H}_{14} \mathrm{O}_{4}\right)$ in slightly alkaline solutions. The reagent, phenolphthalin, is prepared from phenolphthalein by reduction in the presence of $\mathrm{Zn}$ and a strong alkali. In the 1990s, the Hemastix ${ }^{\circledR}$ test (Miles Laboratories) became available as a dipstick. In this test benzidine is replaced by tetramethylbenzidine (TMB) [121, 122].

The first presumptive test based on the fluorescence effect was developed by Fleig in 1910. The fluorescin/fluorescein test is based on the fluorescence emitted by fluorescein when 
is exposed to a light source in the range $425-485 \mathrm{~nm}$. The test uses the capability of heme molecules to catalyze the oxidation of fluorescin to fluorescein $[121,122]$. The chemiluminiscent luminol test, developed by the German forensic scientist Walter Specht (1907-1977) in 1937, is based on the capability of blood to enhance the luminescence of luminol in an alkaline solution of hydrogen peroxide [121, 122]. More recently a derivative of luminol (3-aminophthalhydrazide) has been developed for rapid detection of blood [123].

Currently, it is frequent to use combinations of these tests for presumptive analysis of bloodstains in parallel with the use of alternative light sources or smartphones that incorporate a Raspberry Pi as UV imaging equipment $[121,124]$.

Crystal tests are mainly used as confirmatory tests. The Polish anatomist Ludwik Karol Teichmann (1823-1895) found in 1853 that blood pigmented species such as hemin, a protoporphyrin IX, contains $\mathrm{Fe}^{3+}$ coordinated with chloride ligands. That species, when isolated, forms microscopic crystals with characteristic morphology that confers analytical applications: the crystal Teichmann, Hemin, or Hematin halide test [121, 122]. The Takayama test, developed by the Japanese scientist Masaeo Takayama (1871-1944) in 1912, is a hemochromogen crystal assay based on the formation of red crystals of pyridine ferroprotoporphyrin as result of the interaction between ferrous iron from hemoglobin and pyridine solution. ${ }^{19}$ These tests, together with other crystal tests that use porphyrins such as hematin and acetone chlorhemin tests, have been abandoned [121, 122]. A number of procedures have been developed for applying ELISA as confirmatory test for blood and other biofluids such as semen, sweat, saliva, and tears. In these assays, a variety of blood serum proteins are used as target antigen analytes: human hemoglobin $\mathrm{A} 0$ ( $\mathrm{HbA} 0)$, human leukocyte antigen (HLA), anti-human immunoglobulin $\mathrm{G}(\mathrm{IgG})$, muscle-specific beta-enolase, and human immunoglobulins [125, 126].

Smartphones and microfluidic devices have been introduced as alternative methods for in-field presumptive blood tests: on-site blood sensors based on the colorimetric measurements with a smartphone hardware and software [70] and $\mu \mathrm{PAD}$ methods for the determination of the cause of death and the estimation of postmortem interval [127] are good examples of these novel methods.

Table 4 summarizes the currently most frequently used tests for blood identification [121-126].

After the presence of blood is confirmed, the forensic scientist may perform tests to determine blood individualization. Currently most of these analyses are carried out by means of instrumental techniques that characterize the deoxyribonucleic acid (DNA) of the individual's blood

\footnotetext{
19 The original test by Takayama was improved by A. L. Hatch in 1993 who added dithiothreitol to the Takayama's reagent (Cleland's reagent). This compound reduces the competing effect of oxygen with pyridine for the binding sites in the heme molecule.
}

(human or animal) [123] and ribonucleic acid (RNA) [128]. This technique was used for the first time in 1984 for forensic purposes by the British geneticist Sir Alec John Jeffreys to resolve an immigration case [129]. Nevertheless, some simpler tests were used before this sophisticated technology was implemented. These tests are classified as (a) tests for discriminating blood source or origin (human or animal) and (b) tests for identifying the person's blood.

(a) Tests for identifying blood. The identification of the origin of blood can be performed by identifying specific serum and non-serum proteins. Assays based on serum protein analysis currently are carried out with electrophoretic techniques but some spot test can also be used, which employ immunoprecipitation. This phenomenon, which was independently reported by the German scientists Paul Uhlenhuth (1870-1957) in Greifswald [130, 131] and by August Paul von Wassermann (1866-1925) aided by Albert Schütze, in Berlin in 1901 [132], is based on the formation of a precipitate, in either gel or solution phase, when an antiserum and an antigen are brought into contact. The antiserum is obtained by harvesting the antibodies generated when the immune system of the host species (i.e., a mouse) is inoculated with a foreign serum protein (i.e., from a human). This method reliably and specifically detects the human or animal's blood origin in a dried stain. An example of this methodology is the ring precipitin test in which the simple diffusion between two liquids, namely, the antiserum and the bloodstain extract, is used. The bloodstain extract is poured carefully into a test tube containing the antiserum denser layer. Antibodies and antigens from the respective layers diffuse and a thin precipitate layer is formed in the interphase between the two liquid layers (Fig. 44a). The Ouchterlony double diffusion test, developed by the Swedish bacteriologist and immunologist Örjan Thomas Gunnarson Ouchterlony (1914-2004) in 1949 [133], employs a thin layer of agar gel formed on a Petri plate in which are punched four or six holes or wells around another central well. The antiserum is placed in the central well and the bloodstain extracts in the surrounding wells. After a certain period of incubation at constant temperature the extracts diffuse and immunoprecipitate lines of insoluble antigen-antibody complex are observed in the gel between the wells (Fig. 44b). A staining reagent can be used for better recognition of the immunoprecipitate lines formed.

The identification of human or animal individuals by identifying specific non-serum proteins uses the anti-human hemoglobin owing to the species-specific immunological characteristics of hemoglobin molecules. A. Klein in 1905 [134], L. Hektoen and K. Schul- 
Table 4 Tests for forensic identification of blood [121-126]

\begin{tabular}{|c|c|c|}
\hline Tests & Component identified & Author/year/source \\
\hline \multicolumn{3}{|l|}{ Presumptive tests } \\
\hline \multicolumn{3}{|l|}{ Exposure to light sources } \\
\hline Polilight test (alternative light test) & Whole blood & Margot et al. /1983/[123] \\
\hline Smartphone ultraviolet imaging & & Wilkes et al./2016/[123] \\
\hline \multicolumn{3}{|l|}{ Catalytic tests } \\
\hline Guaiacum/van Deen's test & Hemoglobin & Van Deen/1862/[121, 122] \\
\hline Day's test & & Day/1867/[122] \\
\hline Aloin & & Klunge/1882/[122] \\
\hline Phenolphthalin/Kastle-Meyer test & & $\begin{array}{l}\text { Kastle and Shedd/1901; Meyer/1903; Utz/1903; } \\
\text { Higaki and Philp, 1976/[121, 122] }\end{array}$ \\
\hline Leucomalachite Green & & Adler and Adler/1904/[121, 122] \\
\hline Benzidine/Adler's test & & Adler and Adler/1904/[121, 122] \\
\hline$o$-Tolidine & & Ruttan and Hardisty/1912 /[122] \\
\hline$o$-Toluidine & & Gershenfeld/1939/[122] \\
\hline$o$-Dianisidine & & Owen et al./1958/[122] \\
\hline Tetramethylbenzidine (TMB) $\left(\right.$ Hemastix $\left.^{\circledR}\right)$ & & Holland et al./1974; Garner et al./1976/[121, 122] \\
\hline \multicolumn{3}{|l|}{ Chemiluminiscence and fluorescence tests } \\
\hline Luminol & Hemoglobin & Specht/1937/[121, 122] \\
\hline Luminol (3-aminophthalhydrazide) & & Blum/2009/[124] \\
\hline Fluorescin/fluorescein & & Fleig/1910; Lee et al./1979; Cheesman/1995[121, 122] \\
\hline \multicolumn{3}{|l|}{ Confirmatory tests } \\
\hline \multicolumn{3}{|l|}{ Immunological } \\
\hline $\begin{array}{l}\text { ELISA } \\
\text { ELISA membrane }\end{array}$ & Antibodies & $\begin{array}{l}\text { Fletcher et al./1984/[125] } \\
\mathrm{Wu} \text { and Zaman/2012/[126] }\end{array}$ \\
\hline \multicolumn{3}{|l|}{ Crystal test } \\
\hline Hemin/hematin halide/Teichmann's test & Hemoglobin & Teichmann/1853/[121, 122] \\
\hline Pyridine hemochromogen/Takayama's test & & Takayama/1912; Hatch/1993[121, 122] \\
\hline Acetone chlorhemin/modified Teichmann & & Wagenaar/1935/[121, 122] \\
\hline
\end{tabular}

(a)

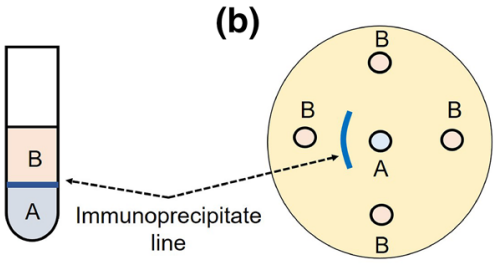

Fig. 44 Scheme of the devices for immunoprecipitation tests. a Ring precipitin test; b Ouchterlony double diffusion test. A antibody solution, B bloodstain extract

hof in 1923 [135], and S. J. Baxter and B. Rees in 1974 [136] successively developed improved tests based on this methodology but H. C. Lee and P. R. DeForest in 1977 [137] developed a more reliable test based on a procedure that enabled the production of a high titer of anti-human hemoglobin serum. Nonetheless, this method exhibited a cross-reactivity with proteins from higher primates and, for this reason, nowadays tests based on IA methodology are preferably used to overcome this setback. LFIAs for discriminating the origin species are based on the affinity between specific monoclonal antibodies for proteins present in red blood cells such as glycophorin A or hemoglobin that act as antigens. M. N. Hochmeinster et al. [138], in 1999, developed the first antigen test for detecting human blood at crime scenes based on an LFIA method, the Hexagon OBTI ${ }^{\circledR}$ one-step test. Currently, improved LFIAs such as RSID ${ }^{\text {TM}}$-Blood (based on glycophorin A antigen) [139] or HemaTrace ${ }^{\circledR}$ ABAcard $^{\circledR}$ (based on human hemoglobin antigen, hHb) [140] are used as presumptive tests [123]. Specific mouse monoclonal antibodies and anti-mouse IgG are used in RSID ${ }^{\mathrm{TM}}$-Blood whereas monoclonal anti-hHb antibodies are used in HemaTrace ${ }^{\circledR}$ ABAcard ${ }^{\circledR}$. 
(b) Tests for identifying the person's blood. The first and most common method for characterizing the individual's blood type is the ABO system. The blood groups are genetic markers of blood of great clinical and forensic interest. The ABO system, widely used worldwide, was discovered by the Austrian biologist and pathologist Karl Landsteiner (1868-1943) awarded with the Nobel Prize in Medicine in 1930. This is a polymorphic blood group system based on the fact that the antigens and the corresponding antibodies have more than one phenotype (type or observable variants). The four types $\mathrm{A}, \mathrm{B}, \mathrm{AB}$, and $\mathrm{O}$ are based on the different structure of the antigen glycoproteins (agglutinogens) A, B, A and $\mathrm{B}$, and $\mathrm{H}$, respectively, present in the membrane of the red blood cells of each group. The corresponding antibodies (agglutinins) are also present in the plasma of each type of blood: anti-B in group A, anti-A in group $\mathrm{B}$, none in group $\mathrm{AB}$, and anti-A and anti-B in group $\mathrm{O}$ [141]. The forensic testing for the ABO markers is focused on the identification of either characteristic antigen and antibody for each group. The analytical methods used are the Lattes crust test, the absorptioninhibition test, and the absorption-elution test. The Lattes crust test or Lattes test was developed in 1913 by the Italian serologist and medico-legal Leone Lattes (1887-1954) [121, 122]. The test consists of exposing three portions of the bloodstain to diluted suspensions of A, B, and O cells. After a suitable period of time, the antibodies are eluted from the stain and undergo an agglutination reaction with the cells, which is observed using a microscope [121]. The absorption-inhibition test was introduced in forensic studies by the Italian forensic scientist Vittorio Siracusa, ${ }^{20}$ in 1923 [142]. In this test the blood type is identified by measuring the strength of a specific antiserum (i.e., anti-B antiserum) after it is brought together with the bloodstain. If the unknown blood contains A antigens, some of the anti-B antibodies of the antiserum will bind to the $\mathrm{A}$ antigens (absorption) and the strength of the original antiserum is decreased (inhibition). This method was replaced by the absorption-elution test, also developed by V. Siracusa, owing to its higher sensitivity [143]. In the absorption-elution test improved by S. S. Kind in 1960 [142], the specific antigens are identified by their agglutination with the homologous antibodies from a small sample of the bloodspot despite the blood cells being lysed in the dry blood. The antigen is immobilized on a solid matrix and then the antibodies are added and absorbed. The unbound antibodies are

${ }^{20} \mathrm{~V}$. Siracusa worked at the Institute of Legal Medicine of the R. University of Messina (Italy). This scientist developed the absorptioninhibition test in collaboration with his mentor L. Lattes. removed by washing and the bound antibodies (anti-A, anti-B, or anti-O antibody) are eluted at high temperature and identified by an agglutination assay using $\mathrm{A}$, B, AB, or O indicator cells (Fig. 45) [121]. This spot test also enables the identification of the $\mathrm{Rh}$ factor, the Rhesus system discovered by the Austrian-US-American biologist, physician, and immunologist Karl Landsteiner (1868-1943, Nobel Prize in 1930) and the US pathologist Alexander Solomon Wiener (1907-1976) in 1940 [121, 122]. Most recently, A and B antigens are rapid and sensitively detected using solid double phase LFIA. The A and B antibodies are immobilized on a nitrocellulose membrane strip. The bound antigens are probed by enzyme-labeled second antibodies of mouse monoclonal type. The dipsticks are applicable not only to blood samples but also to samples of tissues, saliva, urine, or sweat [144]. $\mu$ PADs have been developed for performing in-field identification/analysis of DNA, ABO system, and Rh factor [127]. LFIAs and $\mu$ PAD have been developed for routine clinical analysis but are suitable to assist in forensic analyses.

Saliva: ALS of UV light, high intensity quartz tubes can be used as presumptive test for saliva [123]. The first spot test proposed for identifying saliva was based on the detection of alpha-amylase, secreted by the parotid glands, and responsible for the enzymatic catalysis of breakdown of bonds within the polyglucan chains that compose certain foods. The starch-iodine test was devised by W. Roberts in 1881 [141]. A sample of starch died in blue with iodine is faded when it is brought into contact with a sample of saliva as alpha-amylase acts on starch to break it down and the dye is solubilized. The Phadebas test or Phadebas amylase test (PAT) was developed by G. M. Willott in 1974 [145]. The Phadebas test ${ }^{21}$ is the most commonly presumptive test used for identifying saliva. In the first developed procedure a tablet containing the reagent is brought into contact in a tube test with the liquid sample and the intensity of the color developed in the liquid phase is measured by a spectrophotometer. In 1981 Willott developed the procedure called press test that consists of impregnating filter paper sheets with the solubilized reagent (Procion red amylopectin, PRA). This reagent requires the use of toxic substances such as ethylene glycol monomethyl ether. For this reason, this test has undergone improvements. Currently, the press test is based on microspheres of starch linked to a blue dye that are immobilized on a filter paper sheet. This substrate is digested by the alpha-amylase enzyme of the saliva to release the blue dye proportionally to the amount of alpha-amylase present in

\footnotetext{
${ }^{21}$ Phadebas is the acronym of Pharmacia Diagnostics Biologically Active Substances.
} 


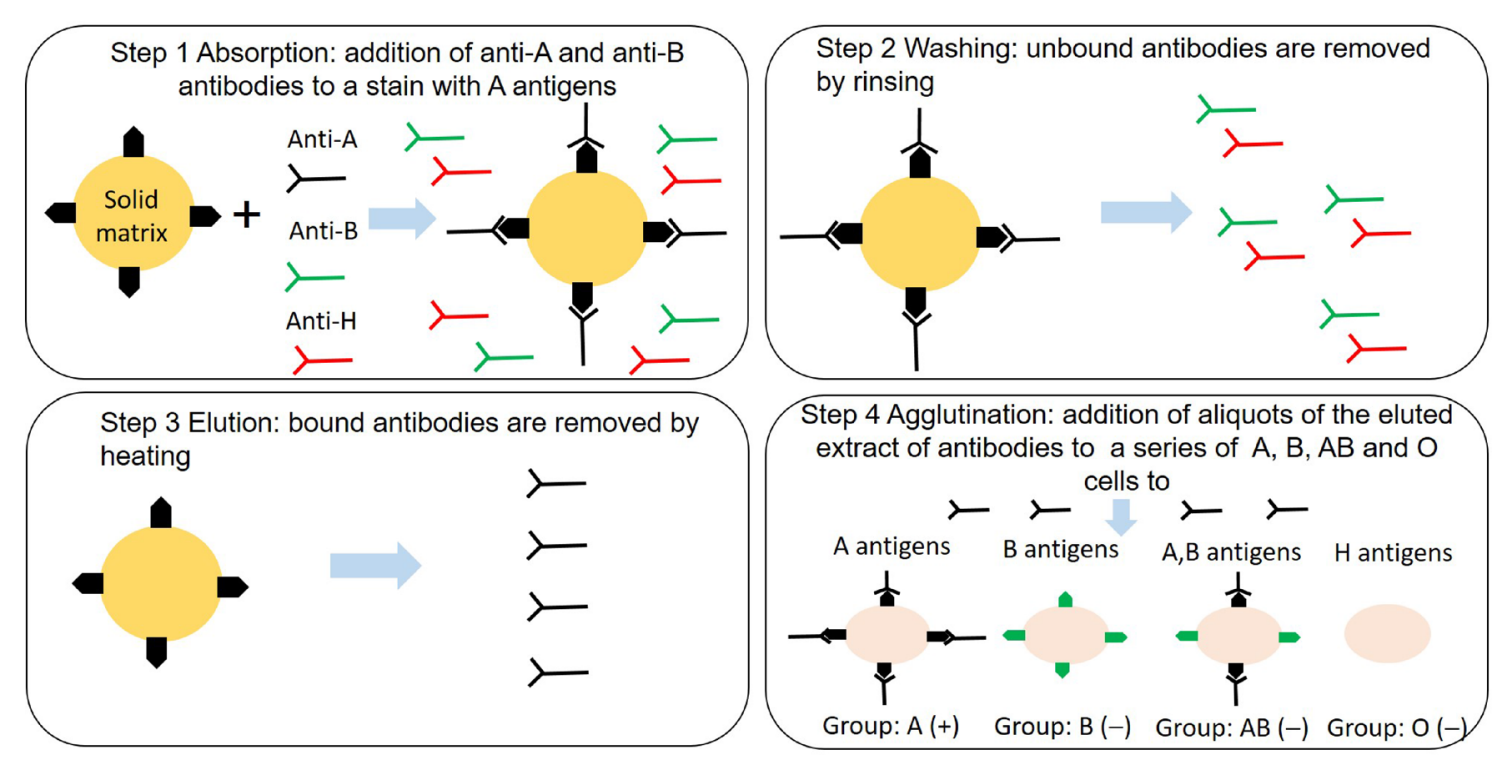

Fig. 45 Scheme of the steps of the absorption-elution test

the sample. A synthetic biochemical substrate with an active component DSM-P, which consists of microspheres containing a chemically cross-linked blue dye, is also used. In addition, Phadebas test is used in hospitals to detect diseases such as acute pancreatitis as well as in the detergent, textile, paper, and food industries. Similar to the Phadebas test is the Amylose Azure test in which the resulting compound of the amylase hydrolysis forms a blue insoluble amylase-dye complex that enables its identification [123]. This test, in combination with a specific LFIA, the RSID ${ }^{\mathrm{TM}^{\mathrm{M}} \text {-saliva test }}$ [146], is used as confirmatory test for human saliva in forensic laboratories.

Semen: Similarly to blood and saliva, there are several spot tests used for presumptive recognition of semen. Seminal acid phosphatase (SAP or AP) catalyzes the hydrolysis of organic phosphates to yield a compound that reacts with a diazonium salt chromogen. The brentamine spot test is based on the detection of the AP enzyme contained in semen by the dark purple color formed in the presence of $\alpha$-naphthyl acid phosphate and brentamine fast blue B. Other less popular spot tests are based on the identification of other enzymes such as leucine aminopeptidase (LAP), glycylproline dipeptidyl aminopeptidase (GDA), cystine aminopeptidase (CAP), and $\gamma$-glutamyltransferase ( $\gamma$-GTP) using Fast Garnet GBC salt and anaphthylamine as indicators. The dipstick test for seminal zinc is another alternative presumptive test [123].

The Christmas tree stain test is a crystal test widely used for confirmation of semen presence in a sample. The identification of sperm cells under a microscope is improved by adding consecutively two staining reagents, picroindigocarmine and nuclear fast red (Kernechtrot), that stain the tail and head of the cellule in blue-green and red, respectively
[147]. Nevertheless, false negatives can be obtained when the individual is azoospermic or was subjected to a vasectomy.

Immunomigration assays commercialized in rapid tests and kit formats have become popular as confirmatory tests for their fastness and ease of use. The LFIA ABAcard or p30 test is based on the screening of prostate specific antigen (PSA) that uses the mobile p30 monoclonal antihuman anti-

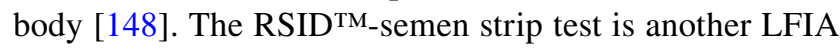
that identifies semengelin, a seminal vesicle-specific antigen [149]. Other rapid tests developed are Biosign ${ }^{\circledR}$ PSA test, Chembio, Medpro, Onco-screen, PSAcheck-1, and Seratec ${ }^{\circledR}$ PSA Semiquant and SMITEST [123].

$\mathrm{Sema}^{\circledR}$ is an ELISA based on the reaction between the seminal vessel-specific antigen (SVSA) also known as $\operatorname{IgG}_{1}$ (MHS-5) and a monoclonal MHS-5 antibody. GGT ELISA is based on the isozyme gamma-glutamyl transpeptidase (GGT), of spermic or testicular nature, present in seminal plasma [123].

Table 5 summarizes the most current tests that have been used for semen and saliva identification [123, 145-149].

\section{Forensic chemistry}

Forensic chemistry is the application of analytical chemistry to the identification of unknown materials found at a crime scene. The subfield forensic toxicology combines toxicology, analytical chemistry, pharmacology, and clinical chemistry to resolve investigations of cases of death, poisoning, and drug abuse. The samples can be residues of drugs or poisons or human fluids and tissues (blood, urine, or hair), which are used to identify illicit or toxic substances. 
Table 5 Tests for forensic identification of semen and saliva

\begin{tabular}{|c|c|c|}
\hline Saliva & Component identified & Source \\
\hline \multicolumn{3}{|l|}{ Presumptive tests } \\
\hline \multicolumn{3}{|l|}{ Exposure to alternative light sources } \\
\hline UV light, high intensity quartz are tubes & Whole fluid & [123] \\
\hline \multicolumn{3}{|l|}{ Chemical } \\
\hline Starch-iodine & Amylase & {$[123]$} \\
\hline Phadebas $^{\circledR}$ & Amylase & {$[123,145]$} \\
\hline Amylose azure & Amylase & {$[123]$} \\
\hline \multicolumn{3}{|l|}{ Immunological } \\
\hline ELISA & Antibodies & [123] \\
\hline \multicolumn{3}{|l|}{ Confirmatory test } \\
\hline \multicolumn{3}{|l|}{ Immunological + chemical } \\
\hline 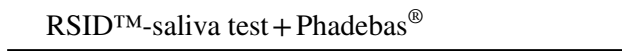 & Antigen & {$[123,146]$} \\
\hline Semen & Component identified & Source \\
\hline \multicolumn{3}{|l|}{ Presumptive tests } \\
\hline \multicolumn{3}{|l|}{ Exposure to alternative light sources } \\
\hline Wood's Lamp, Bluemaxx BM500, Polilight & Whole fluid & {$[123]$} \\
\hline \multicolumn{3}{|l|}{ Chemical tests } \\
\hline Zinc dipstick & Zinc ion & {$[123]$} \\
\hline \multicolumn{3}{|l|}{ Catalytic tests } \\
\hline Acid phosphatase (SAP) & Enzymes & {$[123]$} \\
\hline Leucine aminopeptidase (LAP) & & [123] \\
\hline Glycylproline dipeptidyl aminopeptidase (GDA) & & {$[123]$} \\
\hline Cystine aminopeptidase (CAP) & & {$[123]$} \\
\hline$\gamma$-Glutamyltransferase $(\gamma$-GTP) & & [123] \\
\hline \multicolumn{3}{|l|}{ Confirmatory tests } \\
\hline \multicolumn{3}{|l|}{ Immunological } \\
\hline GGT ELISA & Isozyme & {$[123]$} \\
\hline Sema $^{\circledR}$ & SVSA & [123] \\
\hline ABAcard or p30 test & Prostate specific antigen & {$[123,148]$} \\
\hline RSID $^{\mathrm{TM}}$-semen & Seminal vesicle-specific antigen & {$[123,149]$} \\
\hline \multicolumn{3}{|l|}{ Crystal test } \\
\hline Christmas tree stain & Spermine & {$[123,147]$} \\
\hline
\end{tabular}

Nowadays the majority of confirmatory tests in forensic chemistry use instrumental techniques such as gas chromatography (GC), mass spectrometry (MS), Fourier transform infrared (FTIR) and Raman spectroscopy, nuclear magnetic resonance (NMR), or X-ray diffraction (XRD). Nevertheless, a number of spot tests and crystal tests are employed as presumptive tests together with other instrumental techniques and microscopic examination [150]. Spot tests or color tests are useful as long as they are quick, sensitive, and specific. In particular, in forensic toxicology the sensitivity of a test is given by the limit of detection for a certain drug. The US National Institute of Justice (NIJ) has stated that "a color test's limit of detection is to be defined as ten times the lowest quantity at which a color change occurs five out of five times" (NIJ Standard 0604.01: Color Test Reagents/Kits for
Preliminary Identification of Drugs of Abuse) [151]. Concerning sampling, apart from blood and urine, other body materials such as saliva or hair are used for detecting toxic or illicit substances. Table 6 summarizes some of the most common spot tests used for identifying drugs [151, 152].

Use of crystal tests, like spot tests, has diminished over the years with the incorporation of instrumental techniques. Table 7 summarizes some of the most commonly used [153].

In addition to the classical color and crystal tests, currently one can find a number of commercial portable color test kits for presumptive detection of drugs of abuse in-field (point-of-collection testing, POCT), which have been standardized by the NIJ. The kits are provided in different formats: purity test, test pouches with tube tests and ampoules, sprays and drug wipes, and reagent dropper bottles and plates. The 
Table 6 Spot tests currently used for identifying drugs and poisonous substances [151, 152]

\begin{tabular}{|c|c|c|}
\hline Test & Reagent & Drug identified \\
\hline Liebermann's test & $\begin{array}{l}\text { Sodium nitrite } \\
\text { Sulfuric acid }\end{array}$ & $\begin{array}{l}\text { Alkaloids (cocaine, morphine, etc.): yellow, orange } \\
\text { to brown }\end{array}$ \\
\hline Mandelin's test & $\begin{array}{l}\text { Ammonium metavanadate } \\
\text { Sulfuric acid }\end{array}$ & $\begin{array}{l}\text { Alkaloids (cocaine, morphine, opium, etc.): } \\
\text { orange, red, green, gray, etc. }\end{array}$ \\
\hline Froehde test & $\begin{array}{l}\text { Molybdic acid } \\
\text { Sulfuric acid }\end{array}$ & Aromatic compounds \\
\hline Dille-Koppanyi test & $\begin{array}{l}\text { Cobalt(II) acetate tetrahydrate in methanol } \\
\text { Glacial acetic acid } \\
\text { Isopropylamine in methanol }\end{array}$ & Barbiturates \\
\hline Zwikker test & $\begin{array}{l}\text { Copper(II) sulfate pentahydrate } \\
\text { Pyridine } \\
\text { Chloroform }\end{array}$ & Barbiturates \\
\hline p-DMABA test & $\begin{array}{l}\text { Ethanol } \\
p \text {-Dimethylaminobenzaldehyde } \\
\text { Hydrochloric acid conc. }\end{array}$ & $\begin{array}{l}\text { Benzocaine or procaine: bright yellow } \\
\text { LSD: purple color }\end{array}$ \\
\hline Formaldehyde-sulfuric acid test & $\begin{array}{l}\text { Sulfuric acid conc } \\
\text { Formaldehyde, solution (i.e., } 37 \% \text { formaldehyde) }\end{array}$ & Benzodiazepines: orange color \\
\hline Zimmermann test & $\begin{array}{l}\text { 2,4-Dinitrobenzene in methanol } \\
\text { Potassium hydroxide }\end{array}$ & $\begin{array}{l}\text { Benzodiazepine derivatives and synthetic cathi- } \\
\text { nones }\end{array}$ \\
\hline Duquenois-Levine test & $\begin{array}{l}\text { Vanillin } \\
\text { 95\% Ethanol } \\
\text { Acetaldehyde } \\
\text { Hydrochloric acid conc. } \\
\text { Chloroform } \\
\text { Petroleum ether }\end{array}$ & $\begin{array}{l}\text { Cannabinoids (marijuana, hashish): blue to violet } \\
\text { and purple after adding chloroform }\end{array}$ \\
\hline Fast blue B test & Fast blue B salt (diazotized $o$-dianisidine) & Cannabinoids in cannabis \\
\hline Ehrlich test & $\begin{array}{l}p \text {-Dimethylaminobenzaldehyde in methanol } \\
o \text {-Phosphoric acid }\end{array}$ & Ergot alkaloids and LSD \\
\hline $\mathrm{CO}$ microdiffusion Conway cell test & $\begin{array}{l}\text { Sulfuric acid conc. } \\
\text { Palladium chloride }\end{array}$ & Carbon monoxide \\
\hline Cobalt thiocyanate test & $\begin{array}{l}\text { Cobalt thiocyanate } \\
\text { Deionized water } \\
\text { Hydrochloric acid, acetic acid, or other diluted } \\
\text { acid }\end{array}$ & Cocaine or similar: blue color \\
\hline Scott's test & $\begin{array}{l}\text { Cobalt thiocyanate in glycerin } \\
\text { Hydrochloric acid } \\
\text { Chloroform }\end{array}$ & Cocaine (and methadone) \\
\hline $\mathrm{CO}$ microdiffusion Conway cell test & $\begin{array}{l}\text { Diluted sulfuric acid or tartaric acid } \\
\text { Diluted alkali }\end{array}$ & Cyanide \\
\hline Cyanide color test & $\begin{array}{l}\text { Chloramine-T } \\
\text { Barbituric acid }\end{array}$ & Cyanide \\
\hline Cyanide color test & $\begin{array}{l}p \text {-Nitrobenzaldehide } \\
o \text {-Dinitrobenzene }\end{array}$ & Cyanide \\
\hline Ethanol microdiffusion Conway cell test & $\begin{array}{l}\text { Potassium dichromate } \\
\text { Sulfuric acid }\end{array}$ & Ethanol \\
\hline Ethchlorvynol color test & $\begin{array}{l}\text { Diphenylamine } \\
\text { Sulfuric acid conc. }\end{array}$ & Ethchlorvynol \\
\hline Chen-Kao test & $\begin{array}{l}\text { Acetic acid solution } \\
\text { Copper sulfate solution } \\
\text { Sodium hydroxide solution }\end{array}$ & Ephedrine (and norephedrine) \\
\hline Cobalt nitrite test & $\begin{array}{l}\text { Cobalt nitrate } \\
\text { Isopropylamine } \\
95 \% \text { ethanol }\end{array}$ & $\begin{array}{l}\text { Gamma-hydroxybutyrate }(\mathrm{GHB}+) \text { : purple } \\
\text { Barbiturates }(\mathrm{Barb}+) \text { : purple color by adding } 5 \% \\
\text { isopropylamine in ethanol }\end{array}$ \\
\hline Ferric chloride test & $\begin{array}{l}\text { Ferric chloride hexahydrate } \\
\text { Deionized water or better } \\
\text { Quality-test reagent with GHB or aspirin }\end{array}$ & $\begin{array}{l}\text { GHB: orange-brown } \\
\text { Salicylates: dark purple } \\
\text { Acetaminophen: bluish-gray }\end{array}$ \\
\hline
\end{tabular}


Table 6 (continued)

\begin{tabular}{|c|c|c|}
\hline Test & Reagent & Drug identified \\
\hline Marquis test & $\begin{array}{l}\text { Sulfuric acid } \\
\text { Formaldehyde }\end{array}$ & $\begin{array}{l}\text { Heroin: purple } \\
\text { Methamphetamine: orange-brown } \\
\text { Oxycodone: yellow to brown to violet } \\
\text { Amphetamine: orange-brown } \\
\text { Acetylsalicylic acid: pink-red } \\
\text { Cocaine: salmon }\end{array}$ \\
\hline Forrest test & $\begin{array}{l}\text { Potassium dichromate } \\
\text { Sulfuric acid } \\
\text { Perchloric acid } \\
\text { Nitric acid }\end{array}$ & Imipramine \\
\hline Janovsky test & $\begin{array}{l}m \text {-Dinitrobenzene } \\
\text { Potassium hydroxide } \\
\text { Absolute ethanol }\end{array}$ & $\begin{array}{l}\text { Ketamine: initial brown/purple color with purple } \\
\text { precipitate or specks } \\
\text { Flunitrazepam: strong purple that fades to brown }\end{array}$ \\
\hline Sodium nitroprusside test & $\begin{array}{l}\text { Sodium nitroprusside } \\
\text { Purified water } \\
\text { Acetaldehyde } \\
1 \text { M Sodium hydroxide } \\
1 \text { M Sodium carbonate }\end{array}$ & Methamphetamine: blue color \\
\hline Methanol color test & $\begin{array}{l}\text { Potassium permanganate } \\
\text { Sodium bisulfite } \\
\text { Chromotropic acid } \\
\text { Sulfuric acid }\end{array}$ & Methanol \\
\hline Nitrite color test & $\begin{array}{l}\text { Sulfanilic acid } \\
\text { Hydrochloric acid } \\
\text { Naphthylamine }\end{array}$ & Nitrite \\
\hline Mecke test & $\begin{array}{l}\text { Selenious acid } \\
\text { Sulfuric acid }\end{array}$ & Opium alkaloids \\
\hline Simon's test & $\begin{array}{l}\text { Acetaldehyde in sodium nitroprusside solution } \\
\text { Sodium carbonate }\end{array}$ & Primary and secondary amines \\
\hline Weber test & $\begin{array}{l}\text { Fast blue B (o-dianisidine bis(diazotized) zinc } \\
\text { double salt) } \\
\text { Hydrochloric acid conc. } \\
\text { Deionized water }\end{array}$ & $\begin{array}{l}\text { Psilocin: red color adding the fast blue } \mathrm{B} \text { and blue } \\
\text { after adding } \mathrm{HCl}\end{array}$ \\
\hline Ehrlich's test & $\begin{array}{l}p \text {-Dimethylaminobenzaldehyde } \\
\text { Distilled water } \\
\text { Concentrated hydrochloric acid }\end{array}$ & Psilocybin, psilocin, or LSD: violet \\
\hline Mayer's test & $\begin{array}{l}\text { Mercuric chloride } \\
\text { Distilled water } \\
\text { Potassium Iodide } \\
10 \% \text { Hydrochloric acid (or acetic acid) }\end{array}$ & Tertiary amine: white precipitate \\
\hline
\end{tabular}

most frequently used are NARK ${ }^{\circledR}$ (Sirchie), Narcotic field tests (MMC International BV), NIK ${ }^{\circledR}$ tests $\left(\mathrm{NIK}^{\circledR}\right.$ Public Safety), NarcoTest and NarcoPouch (ODVTM), QuickChech (Lynn Peavey company), EZ test (EZ) and Touch\&Know and TestKitPlus kits supplied by IDenta [151, 154].

ELISA kits are included among the methods used by the International Criminal Police Organization (Interpol) for detecting new psychoactive substances (NPS) in biological samples. Nevertheless, cross-reactivity can reduce the effectivity of the analyses in some cases such as fentanyls, a family of opiate narcotics, as confirmed by recent screening studies [155]. The case of homicidal insulin overdose is a good example of use of ELISA in forensic toxicology. Analysis of insulin is challenging because of the rapid degradation of this molecule in postmortem samples. In such instances, a double-antibody sandwich ELISA has been successfully used [156]. ELISA analyzers are also commonly used as presumptive tests for illicit drugs [157-159]. ELISA and LFIA have been used in the detection of explosives such as trinitroglycerine (TNT) in forensic samples [160].

Smartphone platforms and microfluidic devices have been introduced for testing illicit drugs. Choodum et al. [161-163] have developed a new method based on the application of the DIC to presumptive color tests for illicit drugs such as methamphetamine, amphetamine, and opiates. The digital image can be processed with Adobe PhotoShop or with an application (app) that reads the RGB values of the image. Most accurate analyses can be carried out with pocket 
Table 7 Crystal test commonly used in drug analysis [153]

\begin{tabular}{|c|c|c|c|}
\hline Drug & Reagent & Crystal morphology & Regulation \\
\hline D-Amphetamine & $\mathrm{HAuBr}_{4}, \mathrm{H}_{3} \mathrm{PO}_{4}, \mathrm{H}_{2} \mathrm{SO}_{4}$ & Trapezoidal blades & ASTM E1969-11 \\
\hline D-Amphetamine & $\mathrm{HAuCl}_{4} \cdot 3 \mathrm{H}_{2} \mathrm{O}, \mathrm{H}_{3} \mathrm{PO}_{4}$ & Long yellow rods & ASTM E1969-11 \\
\hline D-Amphetamine & $\mathrm{H}_{2} \mathrm{PtCl}_{6} \cdot 6 \mathrm{H}_{2} \mathrm{O}, \mathrm{H}_{3} \mathrm{PO}_{4}$ & Colorless needles and rods & ASTM E1969-11 \\
\hline DL-Amphetamine & $\mathrm{HAuCl}_{4} \cdot 3 \mathrm{H}_{2} \mathrm{O}, \mathrm{H}_{3} \mathrm{PO}_{4}$ & $\begin{array}{l}\text { Irregular and serrated plates in star or branched- } \\
\text { cluster aggregates and square plates }\end{array}$ & ASTM E1969-11 \\
\hline DL-Amphetamine & $\mathrm{HAuCl} \cdot 3 \mathrm{H}_{2} \mathrm{O}, \mathrm{H}_{3} \mathrm{PO}_{4}$ & $\begin{array}{l}\text { Colorless blades and rods that can aggregate in } \\
\text { rosettes }\end{array}$ & ASTM E1969-11 \\
\hline L-Benzylpiperazine & $\mathrm{H}_{2} \mathrm{PtBr}_{6}$ & $\begin{array}{l}\text { Yellow rectangular and square plates and serrated } \\
\text { X-shaped plates }\end{array}$ & - \\
\hline Clonazepam & $\mathrm{H}_{2} \mathrm{PtCl}_{6} \cdot 6 \mathrm{H}_{2} \mathrm{O}$ & $\begin{array}{l}\text { Blades and rods that can aggregate in fans and } \\
\text { rosettes }\end{array}$ & - \\
\hline Cocaine & $\mathrm{HAuCl}_{4} \cdot 3 \mathrm{H}_{2} \mathrm{O}, \mathrm{HOAc}$ & Long rods with short arms at right angles & ASTM E1968-11 \\
\hline Cocaine & $\mathrm{HAuCl}_{4} \cdot 3 \mathrm{H}_{2} \mathrm{O}, \mathrm{HCl}$ & $\begin{array}{l}\text { Comb-like crosses, individual combs, and clusters } \\
\text { of rods }\end{array}$ & ASTM E1968-11 \\
\hline Cocaine & $\mathrm{H}_{2} \mathrm{PtCl}_{6} \cdot 6 \mathrm{H}_{2} \mathrm{O}, \mathrm{HOAc}$ & Feathery and spiny combs, K- or V-shaped & ASTM E1968-11 \\
\hline Cocaine & $\mathrm{H}_{2} \mathrm{PtCl}_{6} \cdot 6 \mathrm{H}_{2} \mathrm{O}, \mathrm{HCl}$ & Dendritic and feathery, combs, K- and V-shaped & ASTM E1968-11 \\
\hline Codeine & $\begin{array}{l}\text { Marme's reagent-cadmium iodide }\left(\mathrm{CdI}_{2}\right) \text { and } \\
\text { potassium iodide }(\mathrm{KI})\end{array}$ & $\begin{array}{l}\text { Equant tablets, equant and elongated prisms aggre- } \\
\text { gated in rosettes }\end{array}$ & - \\
\hline Codeine & Fulton's O-2 reagent (I-KI), I-KI solution, HOAc & $\begin{array}{l}\text { Hatchet blades, hourglasses, triangles, semicircles, } \\
\text { trapezoids, and rosettes }\end{array}$ & - \\
\hline Codeine & $\begin{array}{l}\text { Fulton's C-3 reagent } \\
\text { I-KI solution, } \mathrm{HOAc}, \mathrm{H}_{2} \mathrm{SO}_{4}\end{array}$ & Triangles and rosettes of rods and triangles & - \\
\hline Diazepam & $\mathrm{H}_{2} \mathrm{PtCl}_{6} \cdot 6 \mathrm{H}_{2} \mathrm{O}$ & Sheaves and rosettes of needles & - \\
\hline Diazepam & $\mathrm{NH}_{4} \mathrm{SCN}$ & Rosettes of thin blades and branched blades & - \\
\hline L-Ephedrine & $\mathrm{HAuBr}_{4}, \mathrm{H}_{2} \mathrm{SO}_{4}$ & Clothespin-shaped needles & - \\
\hline L-Ephedrine & $\mathrm{HAuCl}_{4} 3 \mathrm{H}_{2} \mathrm{O}, \mathrm{H}_{3} \mathrm{PO}_{4}$ & Colorless, elongated plates & - \\
\hline Heroin & $\mathrm{HgCl}_{2}$ & Rosettes of needles & - \\
\hline Heroin & $\mathrm{HgI}_{2}$ & $\begin{array}{l}\text { Colorless needles, fans, branched paddles, and } \\
\text { blades that aggregate in straight-branched or } \\
\text { forked dendrites }\end{array}$ & - \\
\hline Hydrocodone & $\mathrm{H}_{2} \mathrm{PtBr}_{6}, \mathrm{H}_{2} \mathrm{SO}_{4}$ & Needles aggregated in rosettes & - \\
\hline Hydromorphone & $\mathrm{H}_{2} \mathrm{PtBr}_{6}$ & Needles, fans, rosettes, and sheaves & - \\
\hline Hydromorphone & $\mathrm{Na}_{2}\left[\mathrm{Fe}(\mathrm{CN})_{5} \mathrm{NO}\right] \cdot 2 \mathrm{H}_{2} \mathrm{O}$ & $\begin{array}{l}\text { Rods and elongated prisms, aggregated in rosettes } \\
\text { and stars }\end{array}$ & - \\
\hline $\mathrm{MDMA}^{\mathrm{a}}$ & $\mathrm{HAuCl}_{4} \cdot 3 \mathrm{H}_{2} \mathrm{O}, \mathrm{H}_{3} \mathrm{PO}_{4}$ & X-shaped needles & - \\
\hline Methadone & $\mathrm{HgCl}_{2}$ & $\begin{array}{l}\text { Needles and rods aggregated in rosettes, clusters, } \\
\text { burrs, fans, sheaves, and tufts }\end{array}$ & - \\
\hline Methadone & $\mathrm{HgBr}_{2}$ & $\begin{array}{l}\text { Needles aggregated in rosettes, clusters, dendrites, } \\
\text { sheaves, and stars }\end{array}$ & - \\
\hline Morphine & $\mathrm{HAuBr}_{4}, \mathrm{H}_{2} \mathrm{SO}_{4}$ & Clusters, rosettes, and burrs of plates and needles & - \\
\hline Morphine & $\mathrm{HgCl}_{2}$ & Rosettes of needles & - \\
\hline Morphine & Wagner's (I-KI) reagent & Irregular plates & - \\
\hline D-Methamphetamine & $\mathrm{HAuCl}_{4} \cdot 3 \mathrm{H}_{2} \mathrm{O}, \mathrm{H}_{3} \mathrm{PO}_{4}$ & $\begin{array}{l}\text { Clothespins, forked rods, blades, swallow tails, } \\
\text { wheat, and tufts }\end{array}$ & ASTM E1969-11 \\
\hline D-Methamphetamine & $\mathrm{HAuCl}_{4} \cdot 3 \mathrm{H}_{2} \mathrm{O}, \mathrm{H}_{3} \mathrm{PO}_{4}$ & Blunt, rectangular rods narrowed at the ends & ASTM E1969-11 \\
\hline D-Methamphetamine & $\mathrm{H}_{2} \mathrm{PtCl}_{6} \cdot 6 \mathrm{H}_{2} \mathrm{O}, \mathrm{H}_{3} \mathrm{PO}_{4}$ & Dendrites and X-shaped crystals & ASTM E1969-11 \\
\hline Methylphenidate & $\mathrm{PbI}_{2}, \mathrm{HOAc}$ & Rosettes of blades & - \\
\hline Methylphenidate & Picric acid & Rosettes of plates & - \\
\hline Oxycodone & $\mathrm{H}_{2} \mathrm{PtBr}_{6}, \mathrm{H}_{2} \mathrm{SO}_{4}$ & Yellow rosettes of rods and blades & - \\
\hline Oxycodone & Clarke's I-KI, No. 1 & Dark red rods, polygonal shapes, plates and prisms & - \\
\hline Oxycodone & $\mathrm{Na}_{2} \mathrm{CO}_{3}$ & Clusters and stars of rods and X-shaped crystals & - \\
\hline Phencyclidine & $\mathrm{KMnO}_{4}, \mathrm{H}_{3} \mathrm{PO}_{4}$ & $\begin{array}{l}\text { Carmine bow ties, butterflies, squares, rectangles, } \\
\text { and X-shaped crystals }\end{array}$ & - \\
\hline
\end{tabular}


Table 7 (continued)

\begin{tabular}{llll}
\hline Drug & Reagent & Crystal morphology & Regulation \\
\hline Phencyclidine & $\mathrm{NH}_{4} \mathrm{SCN}$ & Rods, blades, and rosettes of blades & - \\
Pseudoephedrine & Dilituric acid & Six-sided plates and tablets & - \\
Pseudoephedrine & $\mathrm{HAuCl}_{4} \cdot 3 \mathrm{H}_{2} \mathrm{O}, \mathrm{H}_{3} \mathrm{PO}_{4}$ & Thin, branching dendrites and combs & - \\
Psilocin & Trinitrobenzoic acid & Rosettes of needles and blade & - \\
\hline
\end{tabular}

${ }^{\text {a }}$ Methylenedioxy- $N$-methamphetamine (ecstasy drug)

colorimetric devices that measure colorimetric coordinates directly on the spot surface [151]. Luminescent upconversion NP-based paper devices for detecting cocaine in saliva have been developed for POCT [164]. $\mu$ PADs have been developed for performing multiplex presumptive color and crystal tests for identification of controlled substances [165]. $\mu$ PADs are also being used progressively for in-field analysis coupled with portable or miniaturized detectors (electrochemical, colorimetric, luminiscence) associated or not with smartphones for identifying explosives, chemical and biological warfare agents, licit drugs, drug counterfeiting, adulterated alcoholic beverages, and milk or food adulterant dyes, drugs of abuse, and cutting agents in drugs of abuse that enable one to understand the drug trafficking. ePADs are used by analyzing drugs of abuse and NPS as well as explosives and gunshot residues [127].

Commercial LOC devices for POCT have been applied for the identification of illicit drugs in samples from clandestine laboratories [166] or from body samples to demonstrate the consumption of drugs of abuse by individuals. A microELISA LOC has been applied to the determination of methamphetamine in human hair. The device is a glass microchip-based ELISA system with microchannels $(200 \times 220 \mu \mathrm{m})$ and a dam structure. The antibody molecules are attached to the surface of polystyrene beads $(45.0 \mu \mathrm{m}$ in diameter) trapped in the microchannel in which is circulated the sample plus the enzyme solution. The liquid sample is obtained from the hair specimen with a micropulverized extraction method. The product of the enzymatic reaction is detected using thermal lens effect that provides an automated full-range quantitation device [167].

It is of worth to separately describe the advances in roadside alcohol and drugs testing. Driving motor vehicles while under the influence of alcohol is another general problem worldwide that has led to governors of most countries to legislate specific laws that prohibit operation of motor vehicles by individuals with blood/alcohol concentration levels over established values. The analysis of individuals suspected of driving under the influence of alcohol is essential for yielding evidence of intoxication and, hence, a law's infraction. The measurement of the blood alcohol content (BAC) that enabled the roadside alcohol testing has been performed for over 100 years until the 1950 s by chemical oxidation with chromic acid and iodimetric titration of ethanol extracted from biological samples [168, 169]. Currently, BAC is conducted by three methods: the handheld breathalyzers determine the alcohol content in exhaled breath by electrochemical oxidation of ethanol to acetic acid with a fuel-cell detector (i.e., Alcolmeter, Alcotest, AlcoSensor, and LifeLoc). The first Breathalyzer was developed by R. F. Borkenstein and H. W. Smith in 1958 [170]. BAC tests in blood samples based on enzymatic methods were also developed in the 1950s. These tests identify the enzyme, alcohol dehyrogenase (ADH) and the phosphatidylethanol (PEth), a phospholipid formed only in the presence of ethanol in blood from the action of phospholipase D [171, 172]. In parallel to spot tests, GC and headspace GC are used as confirmatory tests in breath [173] and blood samples [174], respectively. In urine samples the EtG (ethyl glucuronide) test, which recognizes the presence of the EtG, is commercialized as strip kits [175].

The analysis of oral fluids for driving under the influence of drugs (DUID) started in the 1980s. The first roadside testings (POCT) for drugs in oral fluid were carried out in the 1990s using multiparameter testers [176]. Typical procedures involve sampling of urine, blood, breath, or oral fluid and screening by LFIAs or other IAs followed by confirmatory analysis by GC-MS or high-performance liquid chromatography-mass spectrometry (HPLC-MS) in the laboratory. In particular, a variety of oral fluids or salivary LFIAs for drugs of abuse testing are commercially available, which have been cleared by the US Foods and Drugs Administration [177, 178]. $\mu$ PADs for roadside field testing of DUID have also been developed [164].

Finally, forensic toxicologists are also increasing focused on detecting other compounds of interest for law enforcement, apart from poisonous or toxic illicit drugs, such as pathogens or chemical substances associated with bioterrorism and explosives. For this purpose, the newer generation of spot tests are playing an increasing role.

\section{Clinical and pharmacological sciences}

Clinical chemistry is devoted to the analysis of body fluids. This science started as a subfield of biochemistry in the late nineteenth century with the first uses of chemical 
reactions for testing components of blood and urine for diagnostic and therapeutic purposes. An example is the analysis of creatinine. The German physician Max Jaffe (1841-1911) proposed in 1886 the use of alkaline picrate for the determination of creatinine [179]. This reaction was adapted by the Swedish-born US chemist Otto Knut Olof Folin (1867-1934), in the early twentieth century, into a clinical procedure [180]. In the many decades since, more advanced instrumental techniques have been developed. Nevertheless, analyses based on classic spot tests are still in use for POC testing, which is a medical diagnostic test performed at the place of patient care, or for self-monitoring of the patient at home, owing to their fastness and ease of use. The spot tests and IAs have evolved in the clinical context in a similar way to that already described for forensic sciences. Spot tests based on precipitation and agglutination effects were followed by RIAs and IAs [181]. Spot tests have also evolved from the simpler monoparameter dipsticks such as the Hemastix ${ }^{\circledR}$ test, which is used for the detection of blood in urine as evidence of urinary tract diseases [121], to more complete multiparameter dipsticks. The latter include up to ten different pads with specific reagents that yield a change of color if the test is positive. The analysis is completed in a few minutes after dipping the strip into the fluid to be analyzed. As an example, a commercial urine dipstick includes a spot test for diagnosis of urinary tract diseases and a wide range of other diseases by analyzing leukocytes, nitrite, urobilinogen, protein, $\mathrm{pH}$, hemoglobin, specific gravity, ketone, bilirubin, and glucose. The sodium intake can be determined using chloride dipsticks [182]. In this case, it is assumed that the sodium ingested is mainly due to the salt condiment of food and sodium and chloride are present in equal concentrations in dietary salt.

ELISAs have a wide range of applications for clinical diagnostics: disease markers (cancer, cardiac markers), cell culture and assays, hypersensitivity reactions (allergens and allergic reactions), endocrine system, drug abuse screening, vaccination studies, epidemic and prevention studies, detection of infections, detection of parasites and bacterial toxins. ELISA portable and "ready-to-use" kits are now available that do not require laboratory equipment and skilled personnel. These devices can be used for large population screening at POC [183-186].

The clinical testing for the ABO markers that characterizes the blood type of individuals as described above in forensic serology [141] is an example of the use of LFIAs for clinical analysis. LFIAs is commonly used for self-monitoring at home owing to its ease of use. Good examples are the pregnancy and fertility tests developed in the 1970s $[187,188]$. These tests work by detecting the presence of chorionic gonadotropin (hCG) that is a hormone produced by cells in the placenta during pregnancy and luteinizing hormone made in the pituitary gland that helps control the menstrual cycle or follicle-stimulating hormone to indicate a physiological state in urine. The LFIA Hexagon OBTI test, previously described in the serology section, is a specific test for fecal occult blood testing (FOBT) designed for detecting colorectal cancer disease in either laboratory and POC and was further adapted for forensic blood identification [189]. Quantitative salivary LFIAs have also been developed for detecting cortisol (stress hormone) in sports research [190].

Group A Streptococcus (GAS), also referred to as Group A beta-hemolytic Streptococcus, or Streptococcus pyogenes, are a group of gram-positive bacteria which cause a variety of diseases (pharyngitis, a throat infection), skin infections, glomerulonephritis, sepsis, rheumatic heart disease, toxic shock syndrome, and necrotizing fasciitis. GASspecific LFIAs, based on the detection of Strep A antigen, have been commonly used for professional diagnostics replacing the more conventional and time-consuming agar plate culture method. The sample is throat fluid taken with a cotton swab and the results are provided within $5 \mathrm{~min}$. This is an example of the frequent use of LFIAs for the direct identification of microorganisms [191]. The immunomigration test strip for screening of microalbuminuria is an example of the detection of human diseases in urine [192].

Since the beginning of the severe acute respiratory syndrome coronavirus 2 of the genus Betacoronavirus (SARS$\mathrm{CoV}-2$ ) pandemic, nearly a hundred companies have started to manufacture antigen rapid detection tests as LFIA. The IgG/IgM Rapid Test is a LFIA that detects immunoglobulins $\mathrm{G}(\mathrm{IgG})$ and immunoglobulins $\mathrm{M}(\operatorname{IgM})$ that are antiSARS-CoV-2 antibodies specific for the new strain of coronavirus 2019-nCoV in whole blood, serum, and plasma specimens [193, 194]. This LFIA is a good example of a multiplex site-encoded planar microarray. A scheme of the SARS-CoV-2 LFIA is shown in Fig. 46a. Anti-human IgM and $\mathrm{IgG}$ antibodies are placed in pads or test lines $\mathrm{M}$ and $\mathrm{G}$, respectively, and the anti-rabbit IgG antibody is fixed in control line $\mathrm{C}$ along a nitrocellulose strip. The conjugate pad contains colloidal gold conjugated to recombinant antigen SARS-CoV-2 (Gold SARS-CoV-2 antigen conjugate) and mouse IgG-gold. When a drop of specimen of an infected patient is added to the sample window followed by a drop of assay buffer, anti-SARS-CoV-2 IgM and/or IgG antibodies link to SARS-CoV-2 conjugates forming SARS-CoV-2 conjugate-anti-SARS-CoV-2 antibody complexes. The complexes migrate through the nitrocellulose membrane by capillary action towards the test lines M and G where each complex is fixed forming a burgundy colored SARS-CoV-2 conjugate-anti-SARS-CoV-2 (IgG or IgM) antibody-antihuman (IgM or IgG) antibody complex. The gold rabbit IgG conjugates migrate towards the control pad where they bind to anti-rabbit IgG to form a colored complex and the excess reagents migrate towards the wicking pad [195]. Figure 46b 


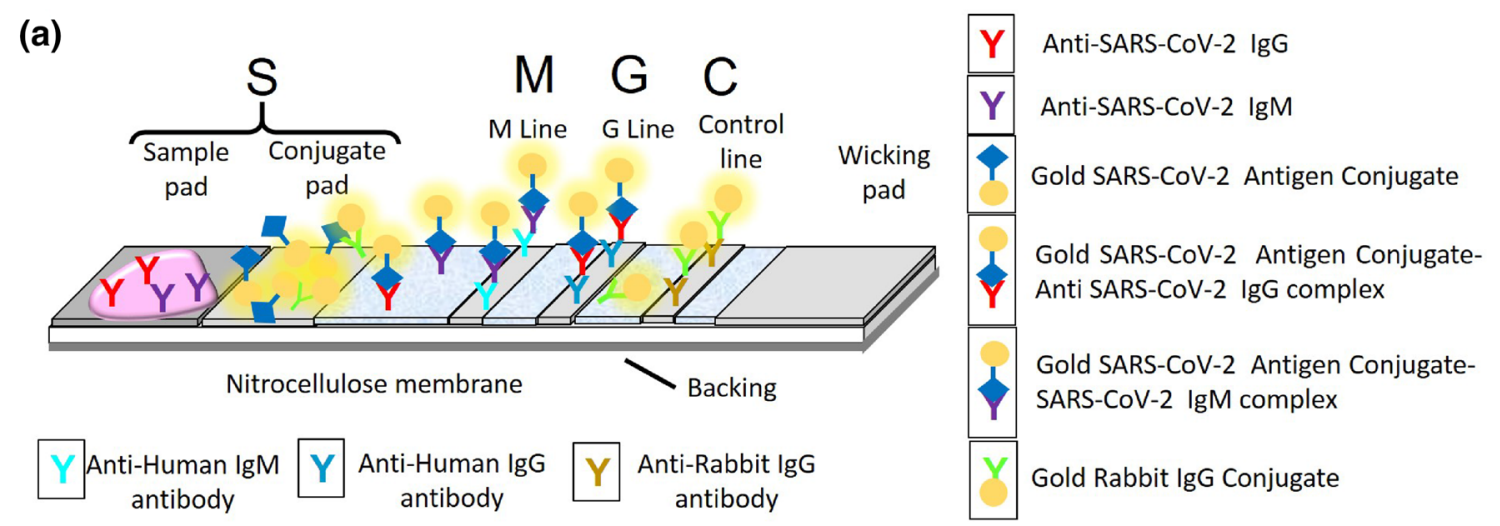

(b)

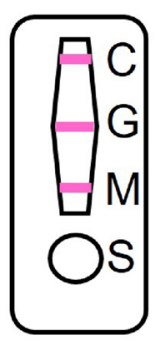

A

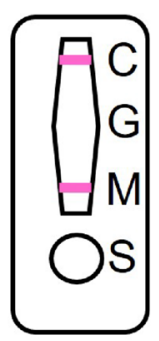

B

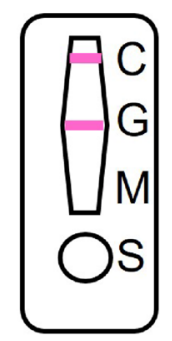

C

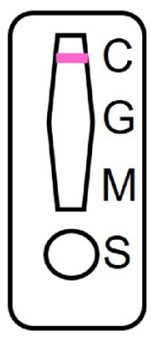

D
Fig. 46 a Scheme showing how the SARS-CoV-2 IgG-IgM combined antibody LFIA works. b Scheme of the SARS-CoV-2 IgG-IgM combined antibody LFIA: A positive result for IgM and IgG antibodies;

shows the different results obtained in this multiplex siteencoded LFIA.

Similarly, most of the rapid drug tests for POC testing in addiction medicine are based on gold-labeled optical-read rapid immunoassay (GLORIA) technology. This LFIA is performed on urine samples and detects antigens specific for each drug by complexation with suitable gold-labeled antibodies [154].

Optical immunoassays (OIA) are a technical advancement of LFIAs. In OIAs the antigen-antibody complexes formed when the assay is positive increase the thickness of the molecular film in the test pad so that the optical path of a reflected light beam also increases and results in a change in the color of the test pad from gold to purple. In most cases, an instrumental register is needed to detect the change in the optical signal [196].

The devices used by patients with diabetes to self-monitor glucose are biosensors that consist of finger sticks based on the enzyme glucose oxidase that reacts with the glucose present in the blood sample and forms gluconic acid. The strip is connected to a meter that provides a readout of the level of blood glucose in the blood depending on the amount of gluconic acid formed. Recently strip tests with dual channels have been developed that enable the simultaneous
B positive result for IgM antibodies; C positive result for IgG antibodies; D negative result for either $\operatorname{IgM}$ and $\mathrm{IgG}$ antibodies. Figure adapted from [195]

monitoring of glucose and other parameters such as uric acid or ketone using smartphones [197].

$\mu$ PADs have also been developed in the field of clinical analysis to overcome the limitations of conventional methods [198, 199]. Naked eye-based detection only provides semiquantitative data. Typical methods to achieve quantitative data of analytes are based on different detection techniques: (a) colorimetric analysis (i.e., DIC using a CIS or CCD-based detector in smartphones, digital cameras, or scanners); (b) luminiscent analysis (i.e., smartphone-based reader platform); (c) magnetic signal detection (i.e., magnetic reader such as magnetic particle quantification (MPQ) reader); electrochemistry-based detection (i.e., glucose meter or analogous reader-based analysis) [92, 93, 199]. Applications of $\mu$ PADs in medical diagnostics cover a variety of disorders and diseases: diabetes, obesity, hypertension, cardiovascular disorders, or cancer and infectious diseases due to pathogens (E. coli, H1N1, Noroviruses) as well as DNA analysis or proteomics [93, 200, 201]. Nanofluidic devices are also being introduced progressively in the clinical diagnostic field in particular, e.g., devices aimed at separating charged species such as DNA on the basis of anodic aluminum and graphene materials [202].

Clinical microfluidic LOC for detecting and quantifying diagnostic markers of varied diseases are already 
commercialized: (a) cardiac injury markers such as myoglobin, creatinine kinase-MB (CK-MB), ${ }^{22}$ troponin I, and B-type natriuretic peptide (BNP); (b) C-reactive protein (CRP) blood test marker of body inflammation diseases; (c) markers of carcinogenic diseases such as PSA, tumor necrosis factor- $\alpha(\mathrm{TNF} \alpha)$, liver cancer marker, $\alpha$-fetoprotein (AFP), and carcinoembryonic antigen (CEA); and (d) markers of infectious diseases such as $\mathrm{CD} 4^{+} \mathrm{T}$ lymphocyte counting for monitoring the human immunodeficiency virus (HIV) and specific markers of dengue fever, influenza A, hepatitis C, malaria, and tuberculosis [203].

Main innovations in POC testing stem from combining the already employed analytical methods with CP-based technologies. Smartphone built-in digital cameras are used for microscopic microimaging. CP-based glucose meters are already available on the market. CP platforms are also used in LFIAs, ELISAs, anti-E. coli O157:H7 Ab-functionalized capillary arrays, urine multiparameter test strips and biochemical assays, among others [201, 204]. An increasing number of methods couple $\mu$ PADs to smartphones [198, 201, 205]. An example of this new methodology is the determination of uric acid in saliva for detecting kidney dysfunctions. The spot test uses the reduction of $\mathrm{Cu}^{2+}$ to $\mathrm{Cu}^{+}$by uric acid and further formation of a violet $\mathrm{Cu}^{+}$complex with 2,2'-biquinoline-4,4'-dicarboxylic acid. Qualitative analysis runs from the digital image acquired with the camera of a smartphone on the colored spot formed placed next to a small color chart. The RGB values are converted with useroptimized software or, alternatively, the images taken with the smartphone camera could be sent to a specialist making use of telemedicine. Quantitative analysis requires "readers" such as image analysis software running on a computer or a smartphone or specialized instruments that detect color coordinates, fluorescence, chemiluminescence, or another optical property of the particles in the test and control lines of the test strip [206]. Another two examples are the determination of salivary cortisol by LFIA using a smartphone to read the colorimetric signal [207] or the chemiluminiscent signal obtained by adding luminol/enhancer/hydrogen peroxide substrate [208]. MicroELISA LOC device equipped with an on-chip thermal lens detection device (TLD) has been used in the on-site analysis of allergen-specific IgE [209]. Human epididymis protein 4 (HE4) marker of ovarian cancer has been detected with ELISA by means of microchip colorimetric smartphone detection [210].

Multiplexed testing has abundant applications for clinical diagnostics. In the laboratory, multiplexed testing is used for determining proteomics of cellular organisms, DNA and

\footnotetext{
${ }^{22}$ The cytosolic creatinine kinase (CK) enzymes consist of two types of subunits: B (brain) and $\mathrm{M}$ (muscle). Thus, there are three isoenzymes: CK-BB, CK-MM, and CK-MB.
}

RNA diagnostics, screening of disease biomarkers from correlated pathologies by determining the concentration of the specific biomolecules in the biosample analyzed, and in cell assays for studying biomolecular processes under diseased conditions. For instance, biomarkers from blood and cerebrospinal fluid markers are applied to the diagnosis of head trauma diseases. In diseases associated with bacterial infections the discrimination between multiple strains or the determination of antibiotic resistance using multiplex platforms reduces response times and informs drug therapy. In general medical practice, multiplexed testing is used for identifying inflammatory markers [105, 106]. MIA devices adapted for POC diagnostics can be useful to detect several respiratory viruses from a single nasopharyngeal swab sample [211]. Detection of ions of heavy metals (e.g., $\mathrm{Hg}^{2+}$, $\mathrm{Pb}^{2+}, \mathrm{Ag}^{+}$, or $\mathrm{Cr}^{3+}$ ) and $\mathrm{S}^{2-}$, which are responsible for diseases, is another application of MIAs [111]. Analyses for the control of health-related ions of electrolytes are performed with multiplexed 3D ePADs. This technique enables simultaneous identification of ions dissolved normally in blood such as $\mathrm{Cl}^{-}, \mathrm{Na}^{+}, \mathrm{K}^{+}$, and $\mathrm{Ca}^{2+}$ that intervene in the cell metabolism and homeostasis (Fig. 47) [212].

Pharmacology, developed in the nineteenth century, is a biomedical science that studies the physiologic activity and therapeutic effects of chemicals. Although narrowly linked to toxicology, pharmacology explores the benefits of chemicals for human health whereas toxicology studies their adverse effects. Therefore, most spot tests used in toxicology have also been employed in pharmacology for characterizing composition of chemicals. Many examples can be found in the literature. Salicylate, the metabolite of aspirin, is recognized by the purple complex formed with the $\mathrm{Fe}^{3+}$ ions from an acidic solution of ferric chloride used as identifying reagent. Quantitative determination of salicylate can also be conducted with a spot test based on the native fluorescence emitted by this compound deposited in the surface of a filter paper using a light source of optical fibers [213]. Similarly, the combination of the new technology of smartphone images with the classical spot tests has been used in pharmacological studies for the determination of carbamazepine, ciprofloxacin, and norfloxacin, the active principles of several chemicals used as analgesics. This assay uses a reagent formulation that combines chromotropic acid, iron chloride, and sulfuric acid [214]. Similarly to clinical analysis, advanced techniques have been applied in the field of pharmacology for detecting active pharmaceutical ingredients and excipients as well as for pharmacokinetic studies. Although other IA techniques are mainly used in the detection of drugs and pharmacokinetic studies [215], some methods can be found in the literature based on the use of LFIA and ELISA [216, 217]. ELISAs have been also applied in medicinal industries, e.g., quality control for antibiotics and veterinary drugs [183]. $\mu$ PADs and LOC methods for 


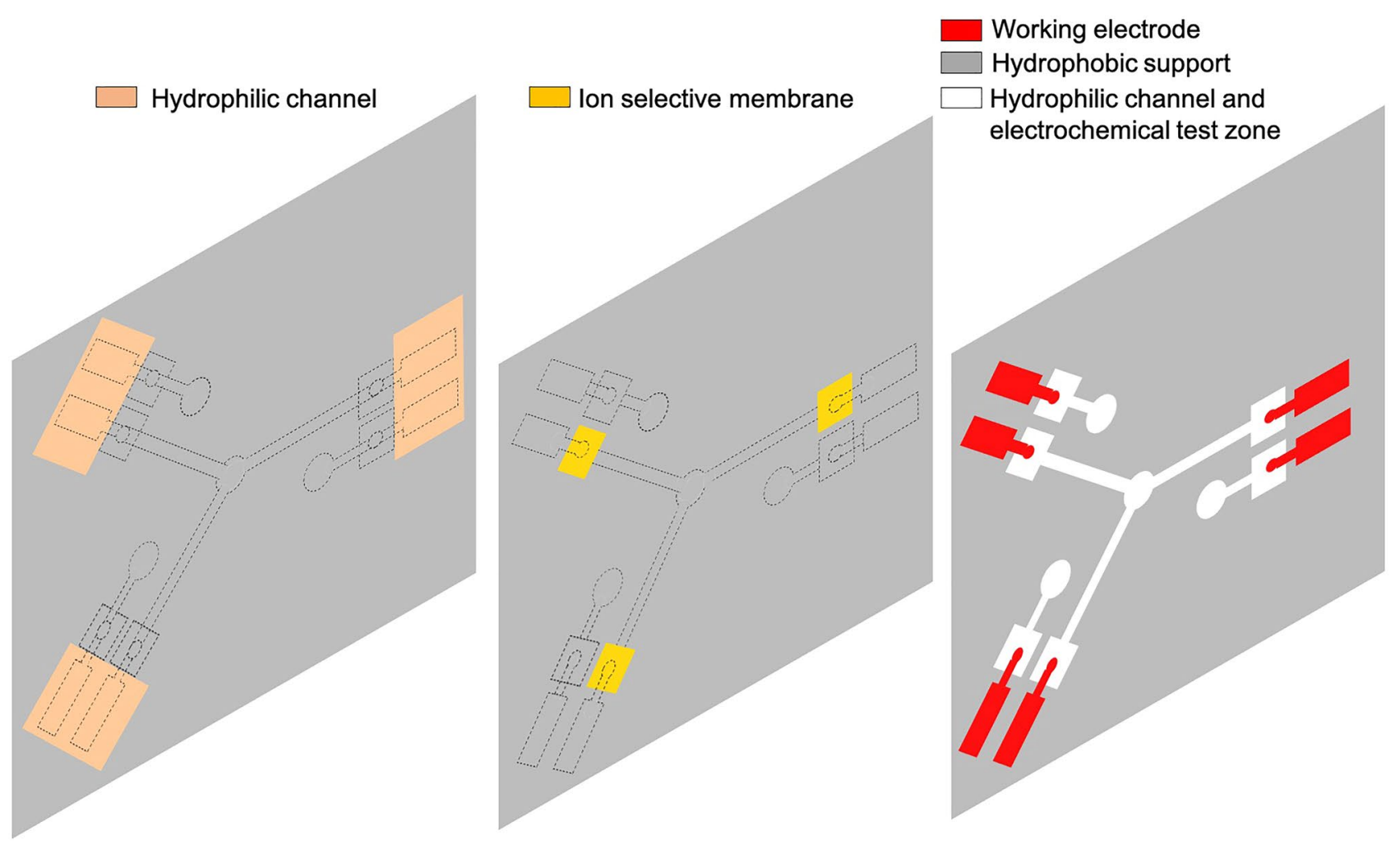

Fig. 47 Scheme of the different layers integrated in a 3D ePAD for simultaneous analysis of blood ions. Adapted from [212]

detection and control of pharmacological substances during the production process as well as in bioanalyses have also been proposed [204, 218, 219]. In particular, several ePADs, also called "pharma-on-chips", have been developed that have as target substances antioxidants, anti-inflammatory and anesthesic drugs, stimulants of the central nervous system, and hormones [219]. In pharmacology multiplexed tests allow drug screening, especially drug resistance, cytotoxicity, and susceptibility as well as drug discovery [105, 106, 111].

\section{Conservation of cultural heritage}

This research field is one in which spot tests were applied very early. The wiping test was frequently used between 1779 and 1809 by historians and antiquarians. This simple test consisted of wiping a small area of a painting with water or organic solvents such as spirits, alcohol, and ether in an attempt to establish the solubility parameters of the varnish or the binding medium and thus its identity [220]. The analysis of the pigments and binding medium of an Egyptian sarcophagus by the German physician and natural scientist Johann Friedrich Gmelin (1748-1804) in 1781 is the earliest account of a spot test of pigments. This scientist conducted the analysis by using reagents, applying heat with a flame and observing changes such as color, smell, etc. [221].
Throughout the nineteenth century, outstanding members of the intellectual elite such as the English chemists Sir Humphry Davy (1778-1829) and Michael Faraday (1791-1867), the French chemists Louis Nicolas Vauquelin (1763-1829), Jean-Baptiste Dumas (1800-1884), and Michel-Eugène Chevreul (1786-1889), the US chemist John William Mallet (1832-1912), the German chemist Friedrich Wilhelm Rathgen (1862-1942) or the German physicist Hans Wilhelm Geiger (1882-1945) were interested in the scientific study of art and archaeological objects [222-224].

The earliest documented account of a spot test conducted on a sample that was prepared as a cross section and observed with a microscope was encountered by the English miniature painter Christopher Barber (1736-1810) in 1783 [220]. The preparation of cross sections as a technique for microscopic examination and recording the stratigraphy of a polychromed or corroded object started with the Scottish chemist Arthur Pillans Laurie (1869-1949) in 1914 and became a standard technique in the 1930s [224]. However, it is in the seminal publication by the English scientist Rosa Joyce Plesters (1927-1996) in 1956 that provided a systematic description of the experimental procedures for conducting spot tests for identifying pigments as well as solvent and staining tests for identifying varnishes and binding media [225]. These tests can be conducted on the mounted sample (Fig. 48) or on the residues of the sample that was left 

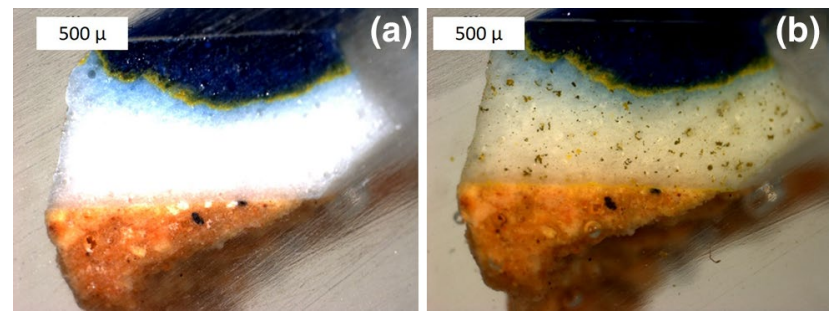

Fig. 48 Cross section of a seventeenth century Spanish majolica ceramic: a lead and tin-glazed ceramic before spot test with potassium iodide solution; $\mathbf{b}$ cross section after successive addition of $\mathrm{HCl}$ and KI drops of reagents. Formation of a yellow precipitate of insoluble lead iodide is concentrated in the bubble cavities formed during the cooling of the ceramic piece after its firing in the furnace
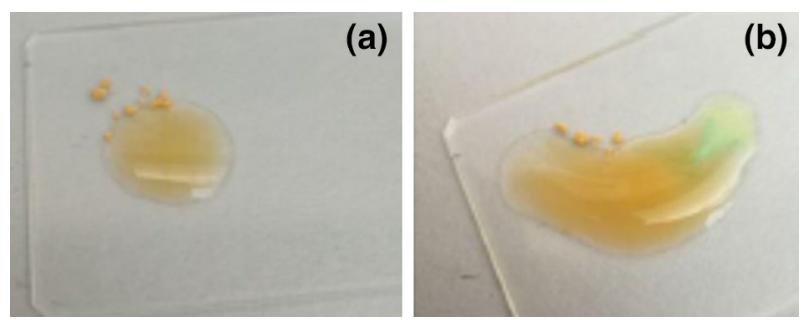

Fig. 49 Unmounted sample of paint: a powdered sample of ochre yellow pigment before spot test with potassium hexacyanoferrate(II) solution; b sample after successive addition of $\mathrm{HCl}$ and potassium hexacyanoferrate(II) drops of reagents. Formation of Prussian blue is observed after diffusion of the solubilized $\mathrm{Fe}^{3+}$ from the acidified solid sample to the drop of iron hexacyanoferrate(II)

unmounted (Fig. 49). A compilation of spot tests proposed for analyzing inorganic pigments in artworks and archaeological objects can be found in the literature [226] and in a more recent ChemTexts contribution by Doménech-Carbó and Osete-Cortina [223]. Arnold [227] has proposed a series of microchemical slide tests based on precipitation reactions for the qualitative analysis of the main ions that make up soluble salts and efflorescences formed as result of alteration process of stones, mortars, stuccoes, or ceramic fabrics of monuments. These tests are based on the works of the US chemists Emile M. Chamot (1868-1950) and Clyde W. Mason (1898-1983) [228], the German chemist Wilhelm L. A. Geilmann (1891-1967) [229], and the Austrian chemist Fritz Feigl (1891-1971) [2]. Table 8 summarizes the ions present in saline systems occurring in monuments as result of alteration processes as well as the solvents and reagents used in the spot tests.

In parallel to the analysis conducted in the laboratory, in situ semiquantitative analysis of soluble salts and efflorescences formed as result of alteration processes of stones, mortars, stuccoes, or ceramic fabrics can be conducted using commercial dipsticks for specific anions or cations (cf. Fig. 8). If only a qualitative in situ identification of chloride anions is required a simple survey kit based on $\mathrm{AgNO}_{3}$ reagent, small flasks, and the light of a smartphone can be used [37].

Organic compounds used as organic pigments and dyes, binding media, protective coatings, or varnishes in artworks as well as those present in wood, textile, and paper supports can also be identified with spot tests as an alternative to the staining tests also employed as an adaptation from the histological tests used in biology for the selective identification of tissues. The advantage of staining and spot tests compared with robust mass spectrometric, chromatographic, or spectroscopic instrumental techniques is their ability to identify the presence of the organic compound in a specific layer. In contrast, they have a lack of specificity as only the type of family (proteins, lipids, polysaccharides, terpenoids, etc.) is provided. Table 9 summarizes the more common tests currently used [230-233].

Several ELISA and immunofluorescence assays have been developed for the detection of proteinaceous materials used as binding media in artworks as well as in archaeological objects and human or animal rests [234-236].
Table 8 Target ions, solvents, and reagents for spot slide test on soluble salts occurring in monuments [227]

\begin{tabular}{|c|c|c|c|}
\hline Target ion & Solvent & Reagent & Compound formed \\
\hline $\mathrm{Na}^{+}$ & $\mathrm{H}_{2} \mathrm{SO}_{4}$ conc. & $\mathrm{Bi}\left(\mathrm{NO}_{3}\right)_{3}$ & $3 \mathrm{Na}_{2} \mathrm{SO}_{4} \cdot 2 \mathrm{Bi}_{2}\left(\mathrm{SO}_{4}\right)_{3} \cdot 2 \mathrm{H}_{2} \mathrm{O}$ \\
\hline $\mathrm{K}^{+}$ & $\mathrm{H}_{2} \mathrm{SO}_{4}$ conc. & $\mathrm{Bi}\left(\mathrm{NO}_{3}\right)_{3}$ & $3 \mathrm{~K}_{2} \mathrm{SO}_{4} \cdot 2 \mathrm{Bi}_{2}\left(\mathrm{SO}_{4}\right)_{3} \cdot 2 \mathrm{H}_{2} \mathrm{O}$ \\
\hline $\mathrm{Ca}^{2+}$ & $\mathrm{H}_{2} \mathrm{SO}_{4} 2 \mathrm{~N}$ & $\mathrm{H}_{2} \mathrm{SO}_{4} 2 \mathrm{~N}$ & $\mathrm{CaSO}_{4} \cdot 2 \mathrm{H}_{2} \mathrm{O}$ \\
\hline $\mathrm{Mg}^{2+}$ & $\begin{array}{l}\mathrm{HCl} 2 \mathrm{~N}, \mathrm{NH}_{4} \mathrm{OH} \\
\mathrm{HCl} 2 \mathrm{~N}\end{array}$ & $\begin{array}{c}\mathrm{NH}_{4} \mathrm{HPO}_{4}, \mathrm{Na}_{2} \mathrm{HPO}_{4} \\
\mathrm{Na}_{2} \mathrm{HPO}_{4}, \mathrm{CsCl}\end{array}$ & 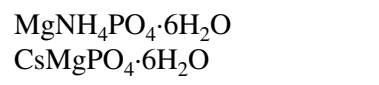 \\
\hline $\mathrm{CO}_{3}{ }^{2-}\left(\mathrm{Ca}^{2+}, \mathrm{Mg}^{2+}\right)$ & $\mathrm{HCl} 2 \mathrm{~N}$ & $\mathrm{HCl} 2 \mathrm{~N}$ & $\mathrm{CO}_{2}(\mathrm{~g})$ \\
\hline $\mathrm{CO}_{3}{ }^{2-}\left(\mathrm{Na}^{+}, \mathrm{K}^{+}\right)$ & $\mathrm{HCl} 2 \mathrm{~N}$ & $\mathrm{pH}$ paper, $\mathrm{CaCl}_{2}$ & $\mathrm{CaCO}_{3}$ \\
\hline $\mathrm{SO}_{4}^{2-}\left(\mathrm{Ca}^{2+},\right)$ & Heat & - & Crystals of gypsum \\
\hline $\mathrm{SO}_{4}^{2-}\left(\mathrm{Mg}^{2+}, \mathrm{Na}^{+}, \mathrm{K}^{+}\right)$ & $\mathrm{HCl} 2 \mathrm{~N}$ & $\mathrm{AgNO}_{3}$ & $\mathrm{AgSO}_{4}$ \\
\hline $\mathrm{NO}_{3}^{-}$ & $\mathrm{H}_{2} \mathrm{SO}_{4}$ conc. & Diphenylamine & Violet diphenylamine complex \\
\hline $\mathrm{Cl}^{-}$ & $\mathrm{H}_{2} \mathrm{NO}_{3} 2 \mathrm{~N}$ & $\mathrm{AgNO}_{3}$ & $\mathrm{AgCl}$ \\
\hline
\end{tabular}


Table 9 Spot tests currently used for identifying binding media, protective coatings, and organic supports [230-233]

\begin{tabular}{|c|c|}
\hline Family or group & Spot test \\
\hline \multicolumn{2}{|l|}{ Binding media, coatings, and varnishes } \\
\hline Proteins & $\begin{array}{l}\text { Biuret test } \\
\text { Sakaguchi test } \\
\text { Millon test } \\
\text { Test of ammonium molybdate test } \\
\text { Ehrlich's reagent (glue in paper) test }\end{array}$ \\
\hline Terpenoid resins & $\begin{array}{l}\text { Storch-Morawski or Lieberman Storch Morawski test } \\
\text { Bromocresol purple test } \\
\text { Raspail (colophony in paper) }\end{array}$ \\
\hline Lipids & $\begin{array}{l}\text { Saponification test } \\
\text { Bromocresol purple (only for waxes and aged drying oils containing free fatty } \\
\text { acids) test }\end{array}$ \\
\hline Synthetic resins & Storch-Morawski or Lieberman-Storch-Morawski test \\
\hline Starch & Lugol test (amylose) \\
\hline \multicolumn{2}{|l|}{ Pigments and dyes } \\
\hline Organic synthetic pigments & $\begin{array}{l}\text { Reagent 1: } \mathrm{H}_{2} \mathrm{SO}_{4} \text { conc.; Reagent 2: } \mathrm{HNO}_{3} \text { conc.; Reagent 3: } \mathrm{H}_{2} \mathrm{SO}_{4} \text { conc./ } / \mathrm{HNO}_{3} \\
\text { conc.; Reagent } 4: \mathrm{KIO}_{3} \text { in } \mathrm{H}_{2} \mathrm{SO}_{4} \text { conc. } \\
\text { Combination of colors formed from the four tests enables identification of fami- } \\
\text { lies and specific organic pigments }\end{array}$ \\
\hline Indigo & Sodium hypochlorite test \\
\hline Cochineal & Reagent 1: $\mathrm{HCl}(1 \%)$; Reagent 2: $\mathrm{NaOH}(1 \%)$; Reagent 3: $\mathrm{NH}_{4} \mathrm{OH}(1 \%)$ \\
\hline Madder & Aluminum sulfate test \\
\hline Redwood & Sulfuric acid test \\
\hline Safflower & Ammonia test \\
\hline Tannic black & Sulfuric acid test \\
\hline \multicolumn{2}{|l|}{ Organic supports } \\
\hline $\begin{array}{l}\text { Cellulose in wood, textile fibers, and paper and other cel- } \\
\text { lulosic adhesives and consolidants }\end{array}$ & Aniline acetate test \\
\hline Lignin in wood, textile fibers, and paper & $\begin{array}{l}\text { Phloroglucinol test } \\
\text { Hertzberg stain test } \\
\text { C-stain test } \\
\text { Aniline sulfate test } \\
p \text {-Nitroaniline test }\end{array}$ \\
\hline Textile fibers (discrimination from animal or vegetal origin) & Sodium azide test \\
\hline
\end{tabular}

\section{Food sciences}

The first applications of analytical chemistry to the study of food date back to the late eighteenth century when the French chemist Antoine Laurent de Lavoisier (1743-1794) measured the $\mathrm{CO}_{2}$ released by human breathing. The first work on nutritional properties of different foods is due to the French surgeon François Magendie (1783-1855) who in 1816 carried out a study of the nutritional properties of different families of food with and without nitrogen content [237]. Several scientists identified a number of substances containing nitrogen present in food from animal and vegetal origin, which were called proteins by the Dutch chemist Gerardus Johannes (Gerrit Jan Mulder) (1802-1880) in 1839 [237]. In the medical field, in addition to physiologic studies, the English physician James Lind (1716-1794) performed in 1746 one of the first clinical trials related to the human diet of sailors at sea. This scientist established more appropriate therapies for avoiding scurvy disease based on the ingestion of certain types of food [237]. These pioneering chemical and clinical studies opened the door to further analytical studies of the nutritional composition of food, paying attention to a series of organic compounds (lipids, proteins, carbohydrates, vitamins, etc.) and chemical elements (Fe, I, $\mathrm{K}, \mathrm{Na}, \mathrm{Ca}, \mathrm{Sr}, \mathrm{Zn}$, etc.) that take part in human metabolism. Spot tests can be applied for this purpose. Nevertheless, these microchemical analyses are of particular relevance in the identification and determination of the content of other compounds and elements that can be incidentally or intentionally present in foods at minor or trace level and are responsible for diseases. Indeed, spot tests play an interesting role in the quality assurance and quality control of food. 
Table 10 Examples of spot tests used for the analysis of common substances present in food [239]

\begin{tabular}{lll}
\hline Test & Reagent & Compound identified \\
\hline Benedict's test & $\begin{array}{c}\text { Cupric sulfate pentahydrated, sodium citrate, sodium carbonate, and sodium } \\
\text { hydroxide }\end{array}$ & Reducing sugars \\
& Solution of potassium iodide with iodine in water & Starch \\
Lugol's iodine test & Sodium hydroxide, hydrated copper(II) sulfate, potassium sodium tartrate & Protein \\
Biuret or Piotrowski's test & Ethanol and water & Lipid \\
Emulsion test & 2,6-Dichloroindophenol, sodium salt & Vitamin C \\
$\begin{array}{l}\text { DCPIP (dichlorophenol-indophe- } \\
\text { nol) test }\end{array}$ & & \\
\hline
\end{tabular}

The growing interest of modern societies in food safety has promoted an increase of the control mechanisms in food traceability $^{23}$ to prevent economic fraudulent practices that result in health troubles; more specifically, in the authenticity control, as an important part of traceability, i.e., in the identification of food components in order to verify that the product complies with the labeling and has a quality warranty.

The presence of adulterants is one of the most common problems detected in quality controls carried out on foods. Adulterants are substances that are substituted or admixed to food, making it unsafe or of lower quality [238]. The latter can also be the result of removing some valuable component. Adulterants are added intentionally (i.e., sand, chalk powder in salt, vegetal oils in milk). Incidentally, a variety of substances can contaminate food (i.e., processing and packaging contaminants such as acrylamides or furans, environmental pollutants such as pesticides, disinfectants, larvae, microorganisms, effluents from chemical industries present in water or in aquatic organisms that have metabolized this water, in particular heavy metals, and agrochemicals such as veterinary drugs). If a food contains a hazardous substance ${ }^{24}$ in excess of the regulatory limit of tolerance it is considered to be adulterated. Although most of the analytical methods of quality control of food are nowadays based on instrumental techniques, spot tests are still today an alternative useful tool because of their simplicity, speed, and low cost. These properties also allow in situ applications. Spot tests are devised for (a) qualitative and quantitative characterization of food components; (b) identification of possible adulterants, environmental or processing contaminants, or substances that do

\footnotetext{
23 The EU General Food Law defines traceability as "the ability to trace and follow a food, feed, food-producing animal or substance intended to be, or expected to be incorporated into a food or feed, through all stages of production, processing, and distribution".

${ }^{24}$ Hazardous substances that can be found in food are classed as foodborne pathogen microorganisms (bacteria, viruses, and fungi) and parasites, biotoxins, or toxic chemical substances produced by animals, plants, and microorganisms, pesticides, veterinary drugs, allergens, heavy metal ions, and unauthorized chemicals.
}

not accomplish the specifications provided by the manufacturer or the government regulations.

Milk is a good example of food subjected to adulteration by addition of water, starch, urea, vegetable oils, formalin, or detergents, among other substances. As an example, a synthetic milk can be prepared by mixing proteins, water paints, oils, alkali, urea, detergents, and glucose or inverted sugar syrup. Sugar can be adulterated with chalk powder, urea, and toxic pigments. Honey is adulterated with sugar solutions. Wheat flour and rice can be adulterated with chalk powder or talc, etc. Tables 10 and 11 summarize some examples of common spot tests employed in the quality control of food [239, 240]. Spot tests based on anthocyanins combined with digital images obtained with smartphones have also been introduced recently for determining acidity of some fruits such as orange, lemon, and passion fruit [241].

Dipsticks are also used in the control of the content of a variety of metals and toxic substances for the quality control of processed drinking water, beer, juices, soft drinks, milk products, and a variety of food. A list of the most frequently analyzed substances has been reviewed recently [43].

Advanced spot testing techniques are widely applied for detecting adulterants and hazardous substances in food and an increasing number of papers can be found in recent literature. Reviews of applications of LFIAs and ELISAs for screening hazardous substances in food have been carried out by Xiao et al. [62] and González-Martínez et al. [89]. The different strategies used for labeling as well as the development of new microfluidic devices and the integration of these analytical devices with smartphone platforms are discussed in the former work. A more specific review focused on detection of hazardous substances in milk has been done by Chen et al. [60]. Similarly, Chen et al. [70] and Kalinowska et al. [76] have dedicated a review to describe the use of novel smartphone platforms with particular incidence in food analysis. Application of multiplexed dipstick, IA, and microfluidic testing has also been reviewed owing to the abundant applications of these innovative methodologies for detecting pharmaceuticals added to feedstuffs 
Table 11 Examples of spot tests used for the analysis of adulterants [240]

\begin{tabular}{ll}
\hline Reagent & Compound identified \\
\hline Sodium potassium tartrate, disulfonic acid, 1-nitroso-2-hydroxynaphthalene-3 & $\mathrm{Co}, \mathrm{Cu}, \mathrm{Fe}$ \\
Sodium hydroxide, benzidine dihydrochloride, acetic acid & $\mathrm{Mn}$ \\
Starch paper, bromine solution & Iodine \\
Potassium hydroxide, iodine, potassium iodide & $\mathrm{Mg}$ \\
Sodium hydroxide, dithiozone, carbon tetrachloride & $\mathrm{Zn}$ \\
Ammonium molybdate, benzidine, acetic acid & $\mathrm{P}$ \\
$\begin{array}{l}\text { Diphenylamine, concentrated sulfuric acid } \\
\text { Sodium picrate, chloroform }\end{array}$ & Nitrites \\
Methanol, $n$-hexane, benzene, anhydrous sodium sulfate, green basic cupric & Cyanogenic glycosides \\
carbonate & Aflatoxin \\
Urease, bromothymol blue, sodium hydroxide & \\
\hline
\end{tabular}

for accelerating growth or preventing diseases in animals for human consumption as well as allergens, mycotoxins, pathogens, melamine, and metal ions, in particular cadmium ions [242-244].

\section{Geosciences}

Most of the qualitative spot tests, also called quick assays, applied in the analysis of rocks and minerals were developed in the eighteenth century by the Swedish naturalists Axel Fredrik Cronstedt (1722-1765), Tobern Olaf Bergman (1735-1784), Johann Gottlieb Gahn (1745-1818), and Jöns Jakob Berzelius (1779-1848). The spot tests developed by Berzelius were elemental analyses that proved the absence or presence of an element in rocks or minerals. In the nineteenth century improvements in spot tests were achieved by several scientists, namely, the German geographer and mineralogist Eduard Harkort (1797-1834), the German mineralogist Franz von Kobell (1803-1882), the German metallurgical chemist Karl Friedrich Plattner (1800-1858), the German chemist Robert Wilhelm Eberhard Bunsen (1811-1899), and the US mineralogist George J. Brush (1831-1912), In the early twentieth century, novel spot tests were developed based on complexation reactions with suitable organic compounds as a result of the great development of organic chemistry. Since the 1960s instrumental techniques such as scanning electron microscopy-Xray microanalysis (SEM-EDX), XRD, inductively coupled plasma torch (ICP) combined with emission spectroscopy (ICP-ES) or MS (ICP-MS), FTIR and Raman spectroscopy that enable quantitative multielemental or mineralogical and crystallographic analysis are the analytical techniques commonly used. Nevertheless, spot tests are still in use for in situ analysis and as a quick and chipper alternative. They require simple arrangements, small amounts of reagents, and a pinch or knife-pointful (50 $\mathrm{mm}^{3}$, this applies to 20 to $60 \mathrm{mg}$ ) [245].
The heating on charcoal test is, most probably, the oldest method for proving ores. Blocks of charcoal $(110 \times 35 \times 12 \mathrm{~mm})$ with a central well are used. Combining the effect of the flame and the burning charcoal, reduction or oxidation of the sample results in the sublimation of the mineral or the volatilization of elements that react with oxygen and form characteristic coatings of oxide on the coal. For instance, if sulfur of sulfides is present, they are oxidized to $\mathrm{SO}_{2}$ with its characteristic odor [245].

A second type of spot tests commonly used in rock and mineral analyses is conducted on solid supports that are heated with a flame. Platinum spoons, wires, and crucibles are used for flame tests. The finely powdered sample is heated in a small hot flame to achieve high temperatures of about $1200{ }^{\circ} \mathrm{C}$. The color of the flame indicates the presence of a chemical element in the sample (e.g., orangeyellow for $\mathrm{Na}$, brick-red for $\mathrm{Ca}$, violet for $\mathrm{K}$, green for $\mathrm{Cu}$, etc.) [245].

In a third type of spot test, the sample is mixed with certain reagents in order to obtain a bead of melt with a characteristic color, a sublimate or a metallic residue specific to an element. Spoons or crucibles of platinum, magnesium rods, or vessels and crucibles of porcelain can be used as support. The melting reagent is borax $\left(\mathrm{Na}_{2} \mathrm{~B}_{4} \mathrm{O}_{7} \cdot 10 \mathrm{H}_{2} \mathrm{O}\right)$, which melts at $742{ }^{\circ} \mathrm{C}$ forming a mixture of sodium metaborate and boric oxide $\left(2 \mathrm{NaBO}_{2}+\mathrm{B}_{2} \mathrm{O}_{3}\right)$. This hot melt reacts with most oxides and silicates and the metal imparts a characteristic color to the bead. On reducing conditions some ions that make up rocks or minerals, such as copper or nickel ions, can be even reduced to the metals. Sodium ammonium hydrogen phosphate tetrahydrate, $\left(\mathrm{Na}\left(\mathrm{NH}_{4}\right) \mathrm{HPO}_{4} \cdot 4 \mathrm{H}_{2} \mathrm{O}\right)$, also called microcosmic salt, can be used for yielding beads at $628{ }^{\circ} \mathrm{C}$ that contain sodium metaphosphate in a glassy state. This melt dissolves most oxides and silicates by the formation of orthophosphates (e.g., $\mathrm{NaCuPO}_{4}$ ) with typical colors [245]. 
Fig. 50 a Scheme of the blowpipe developed by Gahn and first described by Berzelius in 1812 ; $\mathbf{b}$ method of operating the blowpipe

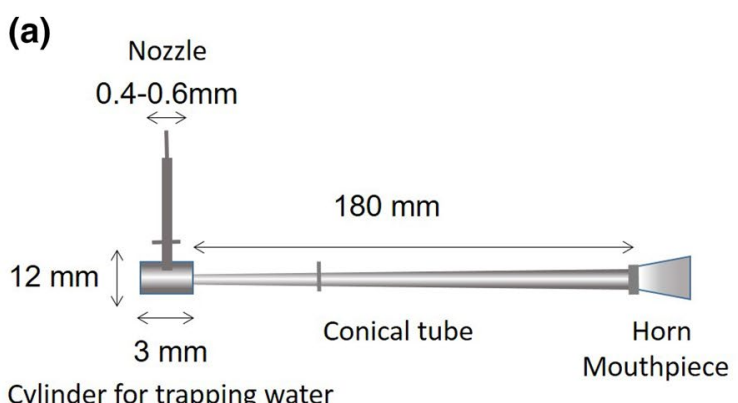

(b)

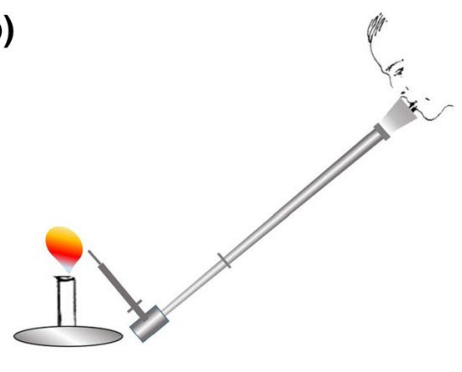

Other classical types of spot tests are conducted in open or closed glass test tubes of 3-6 mm internal diameter and $15-17 \mathrm{~cm}$ or $8-10 \mathrm{~cm}$ length. The produced melt is dissolved and reacts with specific reagents yielding a characteristic colored soluble complex or an insoluble precipitate. This test requires $0.1-20 \mathrm{mg}$ of sample. The sample in the closed tube (a wooden clothes-peg is placed in the open end to seal it after introducing the sample) can change its condition or appearance, form gases which are collected in the tube, or form substances that sublimate or condense on the cold parts of the walls of the tube. In the open tube test, the tube is open at both ends and bent at an angle of $20-30^{\circ}$ to prevent the sample falling out. Complete oxidation of the sample is achieved when the tube is heated with a flame. This test is one of the most important for analyzing minerals of the sulfide group [245].

The heat source used in the aforementioned spot tests has varied throughout centuries. Today Bunsen burners are exclusively used. Nevertheless, in the past hot flames were achieved by a mouth-operated blowpipe and adapted to the flame of a lamp with a wick filled with oil or paraffin (Fig. 50). If the nozzle of the blowpipe was placed outside the lamp a reducing flame was obtained in the end of the blowpipe, whereas an oxidizing flame was obtained by placing the nozzle at one-third within the lamp flame [245].

Although improvements of spot tests or development of new methods has progressively fallen into disuse throughout the twentieth century, some new spot tests have been proposed. For instance, a simple spot test can be performed directly on the surface of the rock for the identification of phosphorus-bearing minerals that uses acid ammonium molybdate to convert the mineral to soluble phosphates, which then form a yellow precipitate [246]. A comparative study of sulfide spot tests for minerals and rocks analysis was carried out by Chan [247]. Another simple spot test for detecting molybdenum minerals uses powdered zinc dithiol as reagent mixed with the powdered sample on a filter paper disk and then a drop of hydrochloric acid is applied resulting in a deep-green color [248]. Experiments with microfluidic devices have been performed for studying pore-scale models of dissolution of calcium sulfate by flowing water and reactive transport in rocks and soils of calcium sulfate that can aid in understanding the behavior during deterioration of building materials such as limestone in polluted environments [249].

\section{Environmental science}

This discipline is characterized by its interdisciplinarity that integrates ecology, biology, physics, chemistry, geology, geography, and social sciences, among others, for the study of the environmental systems and its problems: understanding of earth processes and their interactions with human activities, design of technology for controlling and improving environmental quality, and understanding of human perceptions, relationships, and policies towards the environment. On the other hand, this science has progressively expanded the range of goals owing to the increased social concern for guaranteeing healthy living environments and protecting natural heritage. Indeed, the negative impact of the human activity that generates pollutants and contaminants implies a threat for the natural environments and, concomitantly, contributes to the global climate change. According to Dulio et al. [250] "the term 'contaminants of emerging concern' (CEC) is commonly employed to refer to those chemicals that, in spite of not being currently regulated, might be considered in future regulations due to their potential risk for human health and environment". CEC include pharmaceuticals, personal care products, endocrine disruptors, newly registered pesticides, disinfection by-products, illicit drugs, surfactants, high technology rare earth elements, natural and engineered nanomaterials; CEC refer not only to newly developed compounds but also to those increasingly released to the environment that may be hazardous to the environment, food, and human beings.

This science emerged in the seventeenth and eighteenth centuries in Europe with the philosophical and intellectual movement of the Enlightenment [251]. Among the scientists belonging to this movement was the German naturalist Ernst Heinrich Philipp August Haeckel (1834-1919), who in 1886 introduced the concept of ecology [252]. Nevertheless, the German naturalist Friedrich Wilhelm Heinrich Alexander von Humboldt (1769-1859) can be considered as the first environmentalist. This scientist witnessed how the 
indigenous population of the rainforests of South America extensively burned the forest to transform the soil in cultivation fields and was mindful and warned that human destructiveness had the power to modify the delicate balance of environmental systems [253].

Despite the visionary concept of nature developed by the Enlightenment naturalists, environmental science did not start to be an active science until the 1960-1970s owing to the growing of public awareness for environmental problems and the arrival of environmental laws that established specific protocols of research. As previously described for other application fields, the role of spot tests as an analytical tool for the quality control of environments has been secondary because of the extensive implantation of advanced instrumental techniques in the late twentieth century. Thus, spot tests have been used as a cheap and quick alternative tool for identifying substances which are potentially dangerous for the environment. Hereafter are summarized some examples of application of this classical methodology to different environmental systems and the advances in the spot tests methods that took place in the recent decades.

In the study and control of atmospheric pollution, spot tests have been prevalently used for the analysis of volatile organic compounds (VOC), e.g., for the identification of aliphatic aldehydes by combining a spot test based on the use of 2-hydrazinobenzothiazole as reagent with a colorimetric determination [254]. Another interesting application is the characterization of sizing submicrometer chloride aerosols [255].

Water quality is often controlled by in situ analysis using commercialized dipsticks that provide a semiquantitative determination of a wide number of cations and anions in potable water, wastewater groundwater, surface industrial water, disinfection control, pesticides in agriculture, swimming pool, etc. (see Fig. 8). A list of the most frequently analyzed substances has been reviewed recently [43]. The commercial dipsticks can also be used for analyzing the soil composition. In such instances, a sample of soil, previously weighed, must be properly extracted with a known volume of deionized water and then a drop of the solution is poured onto the pad of the dipstick. Another new method based on fluorimetric sensors applied as test strips enables the identification of ultra-micro-traces of ferric ions in wastewater [256].

Identification of toxic substances present in living beings is of importance for controlling the degree of degradation of ecosystems. For this reason, spot tests developed for this purpose are useful as they can allow rapid in situ analysis $[257,258]$. Similarly, spot tests for in situ identification of toxic substances used in old wall and furniture paints, especially lead compounds, have been extensively used by contractors in charge of the renovation of old buildings where it is suspected that these harmful materials can be present in old paints [259].

Another interesting application of spot tests is found in the field of quality control of new eco-friendly vehicle fuels, the so-called biofuels, obtained from more sustainable sources. A series of methods has been proposed in recent years, which are based on combining spot tests and smartphone digital image evaluation [260,261].

Immunotesting and microfluidic simple or multiplexed tests have also increasingly been used in environmental analysis. LFIA has been used for detecting fungal contamination in work environments. Samples of wall surfaces and airborne dust were taken by wiping clean pieces of filter paper and with an inhalable dust sampler [262]. In environmental sciences MIAs (ELISA, LFIA, fluorescent immunoassays, and biosensors) play a notable role in the detection of a wide range of harmful chemicals such as algal toxins, pesticides, and a variety of multiclass pollutants found in water environments [63]. Pesticides, bacteria, metal ions, and organic substances have also been detected with LFIA [201]. $\mu$ PADs have been used in the detection of pesticides, bacteria, and antibiotics and CECs in water and soils [100, 201, 263, 264]. Dipsticks and ELISA as well as $\mu$ PAD and microfluidic electrochemical sensors that are incorporated into smartphone platforms have been used for detecting metallic ions and other anionic species that contaminate varied natural soil and aquatic environments and wastewater [71, 74, 265-267]. ePADs have been used in studies for occupational exposure to hazardous particulate materials for detecting particulate metals in aerosols [268]. Interest in reducing the time of analysis in environments, where multiple contaminants are suspected to be, such as metal ions present in aquatic environments, has led to the use of multiplexed devices [63, 269].

As it has been presented in the previous sections, NPs (NPs) such as gold ( $\mathrm{Au}$ ), silver ( $\mathrm{Ag})$, zinc ( $\mathrm{Zn})$, and silicon (Si) have been extensively studied not only for applying in detection systems of IAs but also for the removal and detection of pathogenic bacteria from natural environments. Since then, NPs as part of biosensors have received wide attention from many sectors. In comparison with conventional methods, the use of NPs is more cost-effective, time-saving, and safe because of the lack of toxic components [270].

\section{Electrographic testing in practice}

Electrographic printing was applied with success in the middle decades of the twentieth century for qualitative analysis of minor elements present in inorganic and organic materials. Quantification methods were also developed using electrographic transfer combined with a second instrumental technique 
for specifically identifying the metal ions. Nevertheless, the development of more sensitive and fast instrumental techniques that provide multielemental analysis, 2D mappings, and deep profiles of composition were slowly expanding their applications and replacing the electrographic techniques that required the analyst to have a lot of experience and practical skills to obtain suitable results. Among the main applications of this technique in the past was the identification of metal ions composing an unknown metallic material [271]. The electrographic method of Glazunov was applied for qualitative analysis of minor elements present in metals and alloys. The list of minor elements detected is wide: iron, silver, nickel, cobalt, copper, bismuth, zinc, lead, cadmium, tin, antimony, etc. Nevertheless, the scope of this technique was extended soon to other materials such as conducting minerals and ores, detection of the permeability and underfilm spreading of moisture or pore size in organic coatings, and localization of metallic ions in biological materials such as plants and animal tissues [272-275]. Glazunov and Krivohlavy [276] proposed the quantitative analysis of alloys by the electrographic method. Later, several researchers adapted the electrographic printing for quantification of ions by combining it with the ring oven technique, colorimetric or atomic absorption spectroscopy [277].

\section{Addenda: Biography of outstanding spot test scientists}

\section{Fritz Feigl}

Fritz Feigl was born in Vienna in 1891 where he graduated from Technische Hochschule (now Technical University) in 1914. During World War I, he served as an officer in the Austro-Hungarian Army and was wounded in battle and returned as captain with a bronze and a silver medal and the Military Service Cross. After the war, Feigl obtained his doctorate in 1920 with a thesis entitled "Über die Verwendung von Tüpfelreaktionen in der qualitativen Analyse" [5] and was appointed as assistant (1920), docent (1927), professor (1935), and full professor (1937) at the Department of Chemistry of the University of Vienna. Married to Regina, also a brilliant chemist, in 1924, the couple left Vienna with their son in 1938, when Austria was annexed by Germany. They moved to Switzerland and Belgium where, in 1940, Feigl, as Jew, was transferred to a concentration camp near Perpignan. Fortunately, the Brazilian Ambassador in Vichy, Luis Martins de Souza Dantas (1876-1954), obtained the necessary visas for Brazil where the family settled in November 1940. Feigl worked as a researcher at the Laboratorio da Producao Mineral-(LPM), in Rio de Janeiro, where he remained until his death in 1971. Formally retired in 1961, Fritz
Feigl maintained his scientific activity until 1969, when a thrombosis incapacitated him [1, 5, 278, 279].

\section{Aleksandr Ivan'ich Glazunov}

This scientist was born in Saint Petersburg in 1888 into a family that ran a famous publishing house in this city. He studied at the Polytechnic Institute, where obtained in 1912 the degree of an engineer. His carrier started as assistant to the famous Russian chemist Nikolay Semëonovich Kurnakov (1860-1941). During World War I, he was engaged in developing materials for chemical warfare in the Crimea. In 1919, Glazunov was captured by the red partisans and was released after his wife paid a large ransom. He successively moved to the University of Batumi and the Prague Technical University where he defended his PhD project. Between 1923 and 1931, he was assistant professor, associate professor, and full professor of physical chemistry, metallography and alloy theory, electrometallurgy, and ironwork at the Department of Metallurgy of the State College of Mining in Příbram. After this, he became head of the Institute of Theoretical Metallurgy and rector of the State College of Mining. He went back to teaching in 1946 in Ostrava. He became advisor to the Czechoslovak metallurgical industry. Glazunov was considered a reputed expert, member of several editorial boards of scientific journals and international organizations, and was appointed a knight of the French Order of the Legion of Honor. In 1948 he moved to Chile, running away from the communist regime set up in Czechoslovakia. He died in Santiago de Chile in 1951 [280].

Acknowledgements Spanish Project PID2020-113022GB-I00 is supported by MICIN/AEI/10.13039/501100011033. We thank Prof. Mirtat Bouroushian, Athens, for information concerning Georgius P. Skalos.

\section{References}

1. Spinola A, Pinto MS, Costa Neto C (1995) Fritz Feigl (1891-1971). The centennial of a researcher. Bull Hist Chem 17(18):31-39

2. Feigl F, Anger V (1972) Spot tests in inorganic analysis, 6th edn. Elsevier, Amsterdam

3. Jungreis E (1996) Spot test analysis: clinical, environmental, forensic, and geochemical applications, 2nd edn. Wiley, New York

4. IUPAC (1937) Mitteilung der Intemationalen Kommission fur Analytische Reacktionen In: The classification of qualitative analytical reactions, Mikrochim Acta 1:253. The International Commission for New Analytical Reactions and Reagents, (1937) was composed of Bottger W (Leipzig), Feigl F (Wien), Komarowsky AS (Odessa), Van Nieuwenburg CJ (Delft), and Strafford W (Manchester). 
5. Spinola A (2004) Fritz Feigl -Sua obra e novos campos tecnocientíficos pore la originados. Quim Nova 27:169-176

6. Laitinen HA, Ewing GW (eds) (1977) A history of analytical chemistry. Division of Analytical Chemistry, American Chemical Society, Maple Press, York, pp 24-28

7. Delly JG (2020) The literature of classical microchemistry, spot tests, and chemical microscopy. https://www.mccrone.com/theliterature-of-classical-microchemistry-spot-tests-and-chemicalmicroscopy/. Accessed 11 May 2020

8. Raspail FV (1833) Nouveau Système de Chimie Organique, Fonde sur des Methodes Nouvelles d'Observation. Bailliere, Paris

9. Chamot ÉM (1915) Elementary chemical microscopy. Wiley, New York

10. Schiff H (1859) Ann Chim Acta 109:67

11. Emich F (1926) Lehrbuch der Mikrochemie, 2nd edn. Bergmann, Munich

12. Emich F (1931) Mikrochemisches Praktikum. Bergmann, Munich

13. Pregl F (1924) Quantitative organic microanalysis. Translated by Churchill FE from the 2nd German edn, London

14. Emich F (1932) Microchemical laboratory manual, with a section on spot analysis by Fritz Feigl. Translated by Schneider. F Wiley, New York

15. Feigl F (1949) Chemistry of specific, selective and sensitive reactions. Academic, New York

16. Feigl F (1931) Qualitative Analyse mit Hilfe von Tupfelreaktionen. Akademische Verlagsgsellschaft, Leipzig

17. Feigl F (1939) Qualitative analysis by spot tests; inorganic and organic applications. 2nd English edn. Translated from the 3rd German edn. Elsevier, Amsterdam.

18. Feigl F (1966) Spot tests in organic analysis. 7th English edn. In collaboration with Anger V and translated by Oesper RE (previous edns. in 1937, 1939, 1946, 1954, 1956, 1960). Elsevier, Amsterdam

19. Feigl F, Anger V (1972) Spot tests in inorganic analysis. 6th edn, translated by Oesper RE (previous editions in 1937, 1939, 1946, 1954, 1958). Elsevier, Amsterdam

20. Feigl F, Stern R (1921) Über die Verwendung von Tüpfelreaktionen in der qualitativen Analyse. Z Anal Chem 60:1-43

21. Beneke K (1999) Friedrich (Fritz) Feigl und die Geschichte der Chromatographie und der Tüpfelanalyse. Mitt Kolloid-Ges 216-244

22. Feigl F (1923) Tüpfel- und Farbreaktionen als mikrochemische Arbeitsmethoden. (Spotting and color reactions as microchemical techniques). Mikrochemie 1:4-20

23. Schoorl N (1907) Beiträge zur mikrochemischen Analyse. Fresenius' Z Anal Chem 46:658-671

24. Schoorl N (1909) Beiträge zur mikrochemischen Analyse. VIII. Die unlöslichen Substanzen. Fresenius' Z Anal Chem 48:665-678

25. Schoorl N (1908) Beiträge zur mikrochemischen Analyse. II. Analyse der Silbergruppe. Z Anal Chemie 47:209-234

26. Weisz H (1960) Microanalysis by the ring oven technique. Pergamon, London

27. Locke DC, Riley OH (1970) Chemical analysis of paint samples using the Weisz ring oven technique. Stud Conserv 15:94-101

28. Wilson ML (1986) Non destructive rapid identification of metals and alloys by spot tests. Special Technical Publication 550, American Society for Testing and Materials, Philadelphia

29. Gettens RJ, Stout GL (1942) Painting materials. Dover, New York

30. Fardi T, Stefanis E, Panayiotou C, Abbott S, van Loon S (2014) Artwork conservation materials and Hansen solubility parameters: a novel methodology towards critical solvent selection. J Cult Herit 15:583-594
31. Hansen CM (1967) The three dimensional solubility parameter and solvent diffusion coefficient. Danish Technical, Copenhagen

32. Hansen CM (ed) (2007) Hansen solubility parameters. A user's handbook, 2nd edn. CRC, Boca Raton

33. Hildebrand J, Scott RL (1962) Regular solutions. Prentice-Hall, Englewood Cliffs

34. Teas JP (1968) Graphic analysis of resin solubilities. J Paint Technol 40:19-25

35. Yagoda H (1937) Applications of confined spot tests in analytical chemistry: preliminary paper. Ind Eng Chem Anal Ed 9:79-82

36. Wenger PE, Monnier D, Piguet A (1946) Contribution à l'étude d'une méthode d'analyse inorganique semi-quantitative. Helv Chim Acta 29:1698-1701

37. Piechota D, Drake Piechota J (2016) A simple survey kit for chloride detection on cuneïforme tablets and other collections. Stud Conserv 61:58-61

38. Emich F (1917) Über Siedepunktsbestimmung im Kapillarröhrchen. Monatsh Chem 38:219-223

39. Skalos G (1944) Die „Ein“-Tropfen-Reaktion Eine neue Ausführungsform qualitativer Mikroreaktionen. Microchim Acta $32: 233-244$

40. Clarke BL, Hermance HW (1937) Paper as a medium for analytical reactions I. Improvements in the spot test technic. Ind Eng Chem 9:292-294

41. Clarke BL, Hermance HW (1938) Paper as a medium for analytical reactions. A method of applying reagent papers to large volumes of solution. Ind Eng Chem 10:591-600

42. Hopf PP (1946) Chromatographic "spot" tests. J Chem Soc 0:785-786

43. Hillscher LM, Liebich VJ, Avrutina O, Biesalski M, Kolmar H (2021) Functional paper-based materials for diagnostics. ChemTexts 7:14

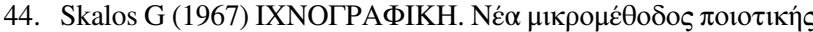
$\alpha \nu \alpha \lambda$ ó $\omega \varsigma$. (Ichnography. A new micromethod of qualitative analysis). Doctor thesis, Chemical Engineering School of NTUA, National Technical University, Athens

45. Conway EJ, Byrne A (1933) An absorption apparatus for the micro-determination of certain volatile substances. The microdetermination of ammonia. Biochem J 27:419-429

46. Masterson B (2013) Conway's microdiffusion analysis: eighty years on and still counting! Biochem J 1:1-7

47. Fritz H (1929) Studies on the sensitivity of some characteristic colour reaction with the help of the electrochemical spot test method. Z Anal Chem 78:418-427

48. Baumann R, Hermance HW, Wadlow HV (1951) Electrography and electro-spot testing. In: Berl WG (ed) Physical methods in chemical analysis, vol II. Academic, New York, pp 155-228

49. Hermance HW, Wadlow HV (1951) Electrography and electrospot testing in physical methods in chemical analysis, vol II. Berl, Academic, New York, pp 155-228

50. Glazunov A (1929) O elektrografickém způsobu reprodukce makrostruktury. In Czech. (On a device for the electrographic reproduction of a macrostructure). Strojnický Obzor (Chim Ind Spec) 24:445-446

51. Shinohara JS, Grasseschi D, Almeida SN, Toma HE (2019) Exploring the metallochromic behavior of pentacyanidoferrates in visual, electronic and Raman spot tests. An Acad Bras Ciênc 91:20180315

52. Kraft A (2018) What a chemistry student should know about the history of Prussian blue. ChemTexts 4:16

53. Pei X, Zhang B, Tang J, Liu B, Lai W, Tang D (2013) Sandwichtype immunosensors and immunoassays exploiting nanostructure labels: a review. Anal Chim Acta 758:1-18 (and references therein)

54. Gründler P (2017) Chemical sensors. ChemTexts 3:16 (and references therein) 
55. Guo J, Chen S, Guo J, Mab X (2021) Nanomaterial labels in lateral flow immunoassays for point-of-care-testing. J Mater Sci Technol 60:90-104 (and references therein)

56. Giokas DL, Vlessidis AG, Tsogas GZ, Evmiridis NP (2010) Nanoparticle-assisted chemiluminescence and its applications in analytical chemistry. Trends Anal Chem 29:1113-1126 (and references therein)

57. Iranifam M (2013) Analytical applications of chemiluminescence-detection systems assisted by magnetic microparticles and nanoparticles. Trends Anal Chem 51:51-70 (and references therein)

58. Richter MM (2008) Electrochemiluminiscence. In: Ligler FS, Taitt ChR (eds) Optical biosensors: today and tomorrow, 2nd edn. Elsevier, Amsterdam (chapter 7 and references therein)

59. González-Carrero S, Pérez-Prieto J (2018) Colloidal photoemissive nanoparticles. ChemTexts 4:8 (and references therein)

60. Chen W, Huang Z, Hu S, Peng J, Liu D, Xiong Y, Xu H, Wei H, Lai W (2019) Advancements in lateral flow immunoassays for screening hazardous substances in milk and milk powder. J Dairy Sci 102:1887-1900 (and references therein)

61. Sanchis S, Singh AK, Senapati D, Neely A, Yu H, Ray PC (2010) Rapid colorimetric identification and targeted photothermallysis of Salmonella bacteria by using bioconjugated oval-shaped gold nanoparticles. Chem A Eur J 16:5600-5606

62. Xiao X, Hu S, Lai X, Peng J, Lai W (2021) Developmental trend of immunoassays for monitoring hazards in food samples: a review. Trends Food Sci Technol 111:68-88 (and references therein)

63. Sanchis A, Salvador JP, Marco MP (2018) Multiplexed immunochemical techniques for the detection of pollutants in aquatic environments. Trends Anal Chem 106:1-10 (and references therein)

64. Das A, Bae K, Park W (2020) Enhancement of upconversion luminescence using photonic nanostructures. Nanophotonics 9:1359-1371 (and references therein)

65. Park JM, Pyun JCh (2015) Chemiluminiscence lateral flow immunoassay based on Pt nanoparticle with peroxidase activity. Anal Chim Acta 853:360-367

66. Yang H, Xu W, Liang X, Yang Y, Zhou Y (2020) Carbon nanotubes in electrochemical, colorimetric, and fluorimetric immunosensors and immunoassays: a review. Microchim Acta 187:206 (and references therein)

67. Creran B, Li X, Duncan B, Chang SK, Moyano DF, Rotello VM (2014) Detection of bacteria using inkjet printed enzymatic test strips. ACS App Mater Interfaces 6:19525-19530

68. Miranda OR, Li X, Garcia-Gonzalez L, Zhu Z-J, Yan BUHF, Rotello VM (2011) Colorimetric bacteria sensing using a supramolecular enzyme-nanoparticle biosensor. J Am Chem Soc 133:9650-9653

69. Huang Y, Wen Y, Baryeh K, Takalkar S, Lund M, Zhang X, Liu G (2017) Lateral flow assay for carbohydrate antigen 19-9 in whole blood by using magnetized carbon nanotubes. Mikrochim Acta 184:4287-4294

70. Chen W, Yao Y, Chen T, Shen W, Tang S, Lee HK (2021) Application of smartphone-based spectroscopy to biosample analysis: a review. Biosens Bioelectron 172:112788 (and references therein)

71. Sivakumar R, Lee NY (2021) Recent progress in smartphonebased techniques for food safety and the detection of heavy metal ions in environmental water. Chemosphere 275:130096 (and references therein)

72. Scholz G, Scholz F (2014) First-order differential equations in chemistry. ChemTexts 1:1

73. Oldham KB, Parnis JM (2017) Shining light on Beer's law. ChemTexts 3:5
74. Delaney JL, Hogan CF, Tian J, Shen W (2011) Electrogenerated chemiluminiscence detection in paper-based microfluidic sensors. Anal Chem 83:1300-1306 (and references therein)

75. Wang X, Gartia RM, Jiang J, Chang T-W, Junle Qian J, Liu Y, Liu X, Liu GL (2015) Audio jack based miniaturized mobile phone electrochemical sensing platform. Sens Actuator B 209:677-685

76. Kalinowska K, Wojnowski W, Tobiszewski M (2021) Smartphones as tools for equitable food quality assessment. Trends Food Sci Technol 111:271-279 (and references therein)

77. Feinberg JG (1956) Agar clarification. Nature 178:1406

78. Feinberg JG (1961) A 'microspot' test for antigens and antibodies. Nature 192:985-986

79. Feinberg JG, Wheeler AW (1963) Detection of auto-immune antibody and tissue antigens by the "microspot" technique. J Clin Pathol 16:282-284

80. Ahsan H, Ahmad R (2020) Multiplex technology for biomarker immunoassays. IntechOpen. http://www.intechopen.com. Accessed 20 Aug 2021

81. Ekins R, Chu F, Biggart E (1990) Fluorescence spectroscopy and its application to a new generation of high sensitivity, multi-microspot, multianalyte, immunoassay. Clin Chim Acta 194:91-114

82. Ekins R, Chu F, Biggart E (1990) Multispot, multianalyte, immunoassay. Ann Biol Clin 48:655-666

83. Yalow RS, Berson S (1959) Assay of plasma insulin in human subjects by immunological methods. Nature 184:1648-1649

84. Yalow RS, Berson S (1960) Immunoassay of endogenous plasma insulin in man. J Clin Invest 39:1157-1175

85. Sathishkumar N, Toley BJ (2021) Paper-microfluidic signalenhanced immunoassays. Prog Mol Biol Transl. https://doi.org/ 10.1016/bs.pmbts.2021.07.015 (and references therein)

86. Engvall E, Perlmann P (1971) Enzyme-linked immunosorbent assay (ELISA). Quantitative assay of immunoglobulin G. Immunochemistry 8:871-874

87. Van Weeman BK, Schuurs AHWM (1971) Immunoassay using antigen-enzyme conjugates. FEBS Lett 15:232-236

88. Sharma A, Gautam S, Bandyopadhyay N (2014) Enzyme immunoassays: overview. In: Batt CA (ed) Encyclopedia of food microbiology, vol 1, 2nd edn. Wiley, New York, pp 680 687 (and references therein)

89. González-Martínez MA, Puchades R, Maquieira A (2018) Immunoanalytical technique: enzyme-linked immunosorbent assay (ELISA). In: Sun DW (ed) Modern techniques for food authentication. Elsevier, Amsterdam (Chapter 15 and references therein)

90. Müller RH, Clegg DL (1949) Automatic paper chromatography. Anal Chem 21:1123-1125

91. Martinez AW, Phillips ST, Butte MJ, Whitesides GM (2007) Patterned paper as a platform for inexpensive low-volume, portable bioassays. Angew Chem Int Ed Engl 46:1318-1320

92. Martinez AW, Martinez NW, Christensen MR (2019) Paperbased methods. Encyclopedia of analytical science, vol 8, 3rd edn. Elsevier, Amsterdam, pp 129-131 (and references therein)

93. Nishat S, Jafry AT, Martinez AW, Awan FR (2021) Paperbased microfluidics: simplified fabrication and assay methods. Sens Actuators B Chem 336:129681 (and references therein)

94. Martinez AW, Phillips ST, Whitesides GM (2008) Threedimensional microfluidic devices fabricated in layered paper and tape. Proc Natl Acad Sci USA 105:19606-19611 (and references therein)

95. Ravgiala RR, Weisburd S, Sleeper R, Martinez A, Rozkiewicz D, Whitesides GM, Hollar KA (2014) Using paper-based diagnostics with high school students to model forensic investigation and colorimetric analysis. J Chem Educ 91:107-111 
96. Tortorich RP, Shamkhalichenar H, Choi JW (2018) Inkjetprinted and paper-based electrochemical sensors. Appl Sci 8:288 (and references therein)

97. Dungchai W, Chailapakul O, Henry CS (2009) Electrochemical detection for paper-based microfluidics. Anal Chem 81:5821-5826

98. Apilux A, Dungchai W, Siangproh W, Praphairaksit N, Henry CS, Chailapakul O (2010) Lab-on-paper with dual electrochemical/colorimetric detection for simultaneous determination of gold and iron. Anal Chem 82:1727-1732

99. León Torres EC, Torrealba Anzola F (2011) The lab-on-achip: existing applications and challeges. Redip Unexpo Vrb Venezuela, Vol 1. http://www.bqto.unexpo.edu.ve/postgrado/ redip. Accessed 18 Aug 2021

100. Pena-Pereira F, Bendicho C, Pavlović DM, Martín-Esteban A, Díaz-Álvarez M, Pan Y, Cooper J, Yang Z, Safarik Y, Pospiskova K, Segundo MA, Psillakis E (2021) Miniaturized analytical methods for determination of environmental contaminants of emerging concern. A review. Anal Chim Acta 1158:238108 (and references therein)

101. Minas G (2008) Lab-on-a-chip devices for chemical analysis. In: Li D (ed) Encyclopedia of microfluidics and nanofluidics. Springer, Boston, pp 910-926

102. Scholz F (2015) Voltammetric techniques of analysis: the essentials. ChemTexts 1:17

103. Mirceski V, Skrzypek S, Stojanov L (2018) Square-wave voltammetry. ChemTexts 4:17

104. Sanjay ST, Dou M, Sun J, Li X (2016) A paper/polymer hybrid microfluidic microplate for rapid quantitative detection of multiple disease biomarkers. Sci Rep 6:30474

105. Araz AK, Tentori M, Herr AE (2013) Microfluidic multiplexing in bioanalyses. J Lab Autom 18:350-366 (and references therein)

106. Goryacheva IY (2016) Rapid multiplex immunotests. In: Goryacheva IY (ed) Rapid immunotests for clinical, food, and environmental applications, vol 72. Comprehensive analytical chemistry series. Elsevier, Amsterdam (Chapter 5 and references therein)

107. Fenton EM, Mascarenas MR, Lopez GP, Sibbett SS (2009) Multiplex lateral-flow test strips fabricated by two-dimensional shaping. ACS Appl Mater Interfaces 1:124-129

108. Liu H, Crooks RM (2011) Three-dimensional paper microfluidic devices assembled using the principles of origami. J Am Chem Soc 133:17564-17566

109. Taranova NA, Berlina AN, Zherdev AV, Dzantiev BB (2015) 'Traffic light' immunochromatographic test based on multicolor quantum dots for the simultaneous detection of several antibiotics in milk. Biosens Bioelectron 63:255-261

110. Du W, Li L, Nichols KP, Nichols K, Ismagilov R (2009) SlipChip. Lab Chip 9:2286-2292

111. Xu Y, Wang H, Chen B, Liu H, Zhao Y (2019) Emerging barcode particles for multiplex bioassays. Sci China Mater 62:289-324 (and references therein)

112. Wilson R, Cossins AR, Spiller DG (2006) Encoded microcarriers for high-throughput multiplexed detection. Angew Chem Int Ed 45:6104-6117

113. Mark D, Haeberle S, Roth G, von Stettenz F, Zengerle R (2010) Microfluidic lab-on-a-chip platforms: requirements, characteristics and applications. Chem Soc Rev 39:1153-1182 (and references therein)

114. Wang C, Hou F, Ma Y (2015) Simultaneous quantitative detection of multiple tumor markers with a rapid and sensitive multicolor quantum dots based immunochromatographic test strip. Biosens Bioelectron 68:156-162
115. Coskun AF, Topkaya SN, Yetisen AK, Cetin AE (2019) Portable multiplex optical assays. Adv Optical Mater 7:1801109 (and references therein)

116. Bell S (2008) Drugs, poisons, and chemistry. Facts on File, New York, pp 8-27

117. Parker RJ (2015) Forensic analysis and DNA in criminal investigations: including cold cases solved. Parker, Toronto, p 66

118. Marsh J (1836) Account of a method of separating small quantities of arsenic from substances with which it may be mixed. Edinb N Philos J 21:229-236

119. Orfila MJB (1827) Du Sang, consideré sous le rapport de la médecine légale; memoire lu à 1'Academie royale de Médecine. J Chim Med Pharm Toxicol 3:365-374

120. Singh R (2020) Narration and legacy of important chemical spot tests in forensic investigation. Crit Rev Anal Chem 12:1-18 (and references therein)

121. Spalding RP (2005) The identification and characterization of blood in bloodstains. In: James SH, Nordby JJ, Bell S (eds) Forensic science: an introduction to scientific and investigative techniques, 2nd edn. Taylor \& Francis, London, pp 237-270 (and references therein)

122. Gaensslen RE (1983) Sourcebook in forensic serology, immunology, and biochemistry (1983) US National Institute of Justice.

123. Virkler K, Lednev IK (2009) Analysis of body fluids for forensic purposes: from laboratory testing to non-destructive rapid confirmatory identification at a crime scene. Forensic Sci Int 188:1-17 (and references therein)

124. Wilkes TC, McGonigle AJ, Pering TD, Taggart AJ, White BS, Bryant RG, Willmott JR (2016) Ultraviolet imaging with low cost smartphone sensors: development and application of a Raspberry Pi-based UV camera. Sensors 16:1649

125. Hurley HP, Cook R, Laughton ChW, Pickles NA, Ireland HE, Williams JHH (2009) Detection of human blood by immunoassay for applications in forensic analysis. Forensic Sci Int 190:91-97

126. Morrison J, Watts G, Hobbs G, Dawnay N (2018) Field-based detection of biological samples for forensic analysis: established techniques, novel tools, and future innovations. Forensic Sci Int 285:147-160 (and references therein)

127. de Araujo WR, Cardoso TMG, da Rocha RG, Santana MHP, Muñoz RAA, Richter EM, Paiãxo TRLC, Coltro WKT (2018) Portable analytical platforms for forensic chemistry: a review. Anal Chim Acta 1034:1-20 (and references therein)

128. Bauer M (2007) RNA in forensic science. Forensic Sci Int Gen 1:69-74 (and references therein)

129. Gill P, Jeffreys A, Werrett DJ (1985) Forensic application of DNA "fingerprints." Nature 318:577-579

130. Uhlenhuth P (1901) Eine Methode zur Unterscheidung der Verschiedener Blutarten, Insbesondere zum Differential-Diagnostischen Nachweis des Menschenblutes. Dtsch Med Wochenschr 27:82

131. Wirth I, Strauch H, Geserick G (2001) Das Uhlenhuth Verfahren. Vorgeschichte in Berlin und erste forensische Gutachten. (The Uhlenhut technique. Prehistory in Berlin and first forensic reports) Rechtsmed 11:217-222

132. Wassermann AP, Schuetze A (1901) Ueber eine Neue Forensische Methode zur Unterscheidung von Menschenand Thierblut. Berl Klin Wochenschr 38:187

133. Ouchterlony O (1949) Antigen-antibody reactions in gels. Acta Pathol Microbiol Scand 26:507-515

134. Klein A (1905) Ueber die Speizifitaet der Erythropraezipitine. Wien Klin Wochenschr 18:1055-1058

135. Hektoen L, Schulhof K (1923) On specific erythroprecipitins (haemoglobin precipitins?): II. Hemoglobin precipitins in identification of blood. J Infect Dis 33:224-229 
136. Baxter SJ, Rees B (1974) The use of antihuman haemoglobin in forensic serology. Med Sci Law 14:159-162

137. Lee HC, DeForest PR (1977) The use of anti-human Hb serum for blodstain identification. In: 29th Annual meeting of the American Academy of Forensic Sciences, San Diego

138. Hochmeister MN, Budowle B, Sparkes R, Rudin O, Gehrig C, Thali M, Schmidt L, Cordier A, Dirnhofer R (1999) Validation studies of an immunochromatographic 1-step test for the forensic identification of human blood. J Forens Sci 44:597-601

139. Technical information and protocol sheet for blood rapid stain identification of human blood (RSID ${ }^{\mathrm{TM}}$-Blood). Independent Forensics DNA Testing \& Technologies. https://www.ifi-test. com. Accessed 18 May 2021

140. HemaTrace ${ }^{\circledR}$ ABAcard $®$ for forensic identification of human blood. Abacus Diagnostics Inc. https://www.abacusdiagnosti cs.com. Technical sheet is available in http://www.rapidtest.cl. Accessed 18 May 2021

141. Greenfield A, Sloan MM (2005) Identification of biological fluids and stains. In: James SH, Nordby JJ, Bell S (eds) Forensic science: an introduction to scientific and investigative techniques, 2nd edn. Taylor \& Francis, London, pp 261-278 (and references therein)

142. Kind SS (1960) Absorption-elution grouping of dried blood smears. Nature 185:397-398

143. Siracusa V (1923) La sostanza isoagglutinable del sangue e la sua dimostrazione per la diagnosi individuale delle macchie. Arch Antropol Crimin Psichiat Med Leg 43:362-365

144. Rao DV, Kashyap VK (1992) A simple dipstick immunoassay for detection of A and B antigens. J Immunoassay 13:15-30

145. Willott GM (1974) A improved test for the detection of salivary amylase in stains. J Forens Sci Soc 14:341-344

146. Technical information and protocol sheet for rapid stain identi-

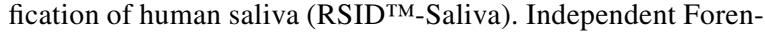
sics DNA Testing \& Technologies. https://www.ifi-test.com. https://www.galantos.eu. Accessed 25 Jul 2021.

147. FB07 Microscopic examination of spermatozoa by Christmas tree, Document Control Number 1577. District of Columbia Department of Forensic Sciences (2014). https://dfs.dc.gov. Accessed 18 May 2021

148. ABAcard or $\mathrm{p} 30$ test for the forensic identification of semen. National Forensic Science Technology Center at Florida International University. http://www.projects.nfstc.org. Accessed 20 May 2021.

149. Rapid stain identification of human semen (RSID ${ }^{\mathrm{TM}}$-Semen) test. Independent Forensics of IL. http://www.ifi-test.com. Accessed 20 May 2021.

150. Cosby DA (2011) Adulterants and interpretive challenges in forensic science: effects on colorimetric spot tests for presumptive drug identification and adverse side effects in the body. PhD Thesis. Boston University. https://hdl.handle.net/2144/14385. http://open.bu.edu. Accessed 2 Apr 2021.

151. Philp M, Fu S (2018) A review of chemical "spot" tests: a presumptive illicit drug identification technique. Drug Test Angl 10:95-108

152. FCS10 Procedure for chemical spot tests, Document Control Number: 7474, District of Columbia Department of Forensic Sciences (2019). https://dfs.dc.gov/publication/fcs10-procedurechemical-spot-tests. Accessed 18 May 2021.

153. Brinsko KM, Golemis D, King MB, Laughlin GJ, Sparenga SB (2016) A modern compendium of microcrystal tests for illicit drugs and diverted pharmaceuticals. McCrone Research Institute, Chicago (and references therein)

154. Wilhelm L (2018) POCT methods for screening in addiction medicine, point-of-care testing. In: Luppa PB, Junker R (eds) Point-of-care testing. Springer, Berlin, pp 171-180 (and references therein)
155. Chan W, Wong GF, Hung Ch, Wong Y, Fung K, Lee W, Dao KL, Leung Ch, Lo K, Lee W, Cheung BK (2016) Interpol review of toxicology. Forensic Sci I 2:563-607 (and references therein)

156. Lv R, Chen Y, Xia N, Liang Y, He Q, Li M, Qi Z, Lu Y, Zhao S (2019) Development of a double-antibody sandwich ELISA for rapid detection to C-peptide in human urine. J Pharmaceut Biomed Anal 162:179-184

157. Elian AA (2003) ELISA detection of clonazepam and 7-aminoclonazepam in whole blood and urine. Forensic Sci Int 134:54-56

158. Kirschbaum KM, Musshoff F, Wilbert A, Röhrich J, Madea B (2011) Direct ELISA kits as a sensitive and selective screening method for abstinence control in urine. Forensic Sci Int 207:66-69

159. Pujol ML, Cirimele V, Tritsch PJ, Villain M, Kintz P (2007) Evaluation of the IDS One-Step ${ }^{\mathrm{TM}}$ ELISA kits for the detection of illicit drugs in hair. Forensic Sci Int 170:189-192

160. Romolo FS, Ferri E, Mirasoli M, D’Elia M, Ripani L, Peluso G, Risoluti R, Maiolini E, Girotti S (2015) Field detection capability of immunochemical assays during criminal investigations involving the use of TNT. Forensic Sci Int 246:25-30

161. Choodum A, Nic DN (2011) Rapid and semi-quantitative presumptive tests for opiate drugs. Talanta 86:284-292

162. Choodum A, Nic DN (2011) Digital image-based colourimetric tests for amphetamine and methylamphetamine. Drug Test Anal 3:277-282

163. Choodum A, Parabun K, Klawach N, Nic Daeid N, Kanatharana $\mathrm{P}$, Wongniramaikul W (2014) Real time quantitative colourimetric test for methamphetamine detection using digital and mobile phone technology. Forensic Sci Int 235:8-13

164. He M, Li Z, Ge Y, Liu Z (2016) Portable upconversion nanoparticles-based paper device for field testing of drug abuse. Anal Chem 88:1530-1534

165. Bell SC, Hanes RDA (2007) Microfluidic device for presumptive testing of controlled substances. Forensic Sci Int 52:884-888

166. Lloyd A, Russell M, Blanes L, Doble P, Roux C (2013) Lab-ona-chip screening of methamphetamine and pseudoephedrine in samples from clandestine laboratories. Forensic Sci Int 228:8-14

167. Miyaguchi H, Takahashi H, Ohashi T, Mawatari K, Iwata YT, Inoue H, Kitamori T (2009) Rapid analysis of methamphetamine in hair by micropulverized extraction and microchip-based competitive ELISA. Forensic Sci Int 184:1-5

168. Widmark EMP (1922) Eine mikromethode zur bestimmung von äthylalkohol im blut. Biochem Z 131:473-484

169. Friedemann TE, Dubowski KM (1959) Chemical testing procedures for the determination of ethyl alcohol. J Am Med Assoc 170:47-71

170. Borkenstein RF, Smith HW (1961) The Breathalyzer and its applications. Med Sci Laws 2:13-22

171. Brink NC, Bonnichsen R, Theorell H (1954) A modified method for the enzymatic microdetermination of ethanol. Scan J Clin Lab Inv 10:223-236

172. Lunquist F, Wolthers $H$ (1958) The kinetics of ethanol elimination in man. Acta Pharmacol Tox 14:265-289

173. Dubowski MK (1980) Alcohol determination in the clinical laboratory. Am J Clin Pathol 74:747-750

174. Jones AW (2019) Alcohol its analysis in blood and breath for forensic purposes, impairment effects, and acute toxicity. WIREs Forensic Sci 1:e1353

175. Schmitt G, Aderjan R, Keller T, Wu M (1995) Ethyl glucuronide: an unusual ethanol metabolite in humans. synthesis, analytical data, and determination in serum and urine. J Anal Toxicol 19:91-94

176. Verstraete AG (2005) Oral fluid testing for driving under the influence of drugs: history, recent progress and remaining challenges. Forensic Sci Int 150:143-150 
177. Jehanli A, Moore L, Brannan S, Spiehler VR (2001) Blind trials of an onsite saliva drug test for marijuana and opiates. J Forensic Sci 46(5):1214-1220

178. Scherer JN, Schuch JB, Rabelo-da-Ponte FD, Silvestrin R, Ornella R, Sousa T, Limberger RP, Pechanskya F (2020) Analytical reliability of four oral fluid point-of-collection testing devices for drug detection in drivers. Forensic Sci Int 315:110434

179. Berger D (1999) A brief history of medical diagnosis and the birth of the clinical laboratory Part 1-Ancient times through the 19th century. Med Lab Obs 31(7):28-30; 32:34-40

180. Berger D (1999) A brief history of medical diagnosis and the birth of the clinical laboratory Part 2-Laboratory science and professional certification in the 20th century. Med Lab Obs $31: 32-34(36,38)$

181. Goryacheva IY (2016) Rapid tests progress through the years. In: Goryacheva IY (ed) Rapid immunotests for clinical, food, and environmental applications, vol 72. Comprehensive analytical chemistry series. Elsevier, Amsterdam (chapter 2)

182. Heeney ND, Lee RH, Hockin BCD, Clarke DC, Sanatani S, Armstrong K, Sedlak T, Claydon VE (2021) At-home determination of 24-h urine sodium excretion: validation of chloride test strips and multiple spot samples. Auton Neurosci-Basic 233:102797

183. Gan SD, Patel KR (2013) Enzyme immunoassay and enzymelinked immunosorbent assay. J Invest Dermatol 133:e12

184. Balsam J, Ossandon M, Bruck HA, Lubensky I, Rasooly A (2013) Low-cost technologies for medical diagnostics in lowresource settings. Expert Opin Med Diagn 7:243-255

185. Mariani M, Luzzi E, Proietti D, Mancianti S, Casini D, Costantino P, van Gageldonk P, Berbers G (1998) A competitive enzyme-linked immunosorbent assay for measuring the levels of serum antibody to Haemophilus influenzae type b. Clin Diagn Lab Immunol 5:667-674

186. Dobrovolskaia E, Gam A, Slater JE (2006) Competition enzymelinked immunosorbant assay (ELISA) can be a sensitive method for the specific detection of small quantities of allergen in a complex mixture. Clin Exp Allergy 36:525-530

187. Heap RB, Holdworth RJ, Gadsby JE, Laing JA, Walters DE (1976) Pregnancy diagnosis in the cow from milk progesterone concentration. Br Vet J 132:445-464

188. Dobson H, Fitzpatrick RJ (1976) Clinical application of the progesterone-in-milk test. Br Vet J 132:538-542

189. Hexagon OBTI Immunochromatographic 1-step test for occult blood in stool. (2021). Human mbH. http://www.human.de. Accessed 23 Jul 2021.

190. Miočević O, Cole CR, Laughlin MJ, Buck RL, Slowey PD, Shirtcliff AS (2017) Quantitative lateral flow assays for salivary biomarker assessment: a review. Front Public Health 5:133 (and references therein)

191. Strep a rapid test strip (throat swab) (2021) Gima professional medical products. Gima SL. http://gimaitaly.com. Accessed 18 Jul 2021.

192. Omidfar K, Kia S, Larijani B (2011) Development of a colloidal gold-based immunochromatographic test strip for screening of microalbuminuria. Hybridoma 30:117-124

193. Antigen-detection in the diagnosis of SARS-CoV-2 infection using rapid immunoassays (2020) World Health Organization. https://who.int. Accessed 19 Jul 2021.

194. David A, Scott L, Jugwanth S, Gededzha M, Kahamba T, Zwane N, Mampeule N, Sanne I, Stevens W, Mayne ES (2021) Operational characteristics of 30 lateral flow immunoassays used to identify COVID-19 immune response. J Immunol Methods 496:113096

195. Li Z, Yi Y, Luo X, Xiong N, Liu Y, Li S, Sun R, Wang Y, Hu B, Chen W, Zhang Y, Wang J, Huang B, Lin Y, Yang J, Cai W, Wang X, Cheng J, Chen Z, Sun K, Pan W, Zhan Z, Chen L, Ye F (2020) Development and clinical application of a rapid IgM-IgG combined antibody test for SARS-CoV-2 infection diagnosis. J Med Virol 92:1518-1524

196. Park ChH, Ruprai D, Vandel NM, Hixon DL, Mecklenburg FE (1996) Rapid detection of group B streptococcal antigen from vaginal specimens using a new optical immunoassay technique. Diagn Microbiol Infect Dis 24:125-128

197. Guo J, Huang X, Ma X (2018) Clinical identification of diabetic ketosis/diabetic ketoacidosis acid by electrochemical dual channel test strip with medical smartphone. Sens Actuat B-Chem 275:446-450

198. Mahato K, Purohit B, Kumar A, Chandra P (2020) Paper-based biosensors for clinical and biomedical applications: emerging engineering concepts and challenges. In: Mercoçi A (ed) Comprehensive analytical chemistry handbook, Vol 89, Paper based Sensors. Elsevier, Amsterdam (and references therein)

199. Yang J, Wang K, Xu H, Yan W, Jin Q, Cui D (2019) Detection platforms for point-of-care testing based on colorimetric, luminescent and magnetic assays: a review. Talanta 202:96-110 (and references therein)

200. Yager P, Edwards T, Fu E, Helton K, Nelson K, Tam MR, Weigl BH (2006) Microfluidic diagnostic technologies for global public health. Nature 442:412-418

201. Li F, You M, Li X, Hu J, Liu Ch, Gong Y, Yang H, Xu F (2020) Paper-based point-of-care immunoassays: recent advances and emerging trends. Biotechnol Adv 39:107442 (and references therein)

202. Resmi PE, Suneesh PV, Ramachandran T, Satheesh Babu TG (2021) Paper based micro/nanofluidics devices for biomedical applications. Pro Mol Biol Transl Sci. https://doi.org/10.1016/ bs.pmbts.2021.07.011 (and references therein)

203. Rattle S, Hofmann O, Price ChP, Kricka LJ, Wild D (2013) Lab-on-a-chip, micro- and nanoscale immunoassay systems, and microarrays. In: Wild D (ed) Immunoassay handbook. Theory and applications of ligand binding, ELISA and related techniques. Elsevier, Amsterdam (and references therein)

204. Vashist SK, Luong JHT (2019) Point-of-care technologies enabling next-generation healthcare monitoring and management. Springer, New York, pp 117-132 (and references therein)

205. Kim DW, Jeong KY, Yoon HC (2019) Smartphone-based medical diagnostics with microfluidic devices. In: Yoon JY (ed) Smartphone based medical diagnostics. Elsevier, Amsterdam (and references therein)

206. Soares S, Rocha FRR (2021) Spot test for determination of uric acid in saliva by smartphone-based digital images: a new proposal for detecting kidney dysfunctions. Microchem J $162: 105862$

207. Choi S, Kim S, Yang JS, Lee JH, Joo C, Jung HI (2014) Realtime measurement of human salivary cortisol for the assessment of psychological stress using a smartphone. Sens Biosensing Res 2:8-11

208. Zangheri M, Cevenini L, Anfossi L, Baggiani C, Simoni P, Di Nardo F, Roda A (2015) A simple and compact smartphone accessory for quantitative chemiluminescence-based lateral flow immunoassay for salivary cortisol detection. Biosens Bioelectron 64:63-68

209. Ohashi T, Matsuoka Y, Mawatari K, Kitaoka M, Enomoto T, Kitamori T (2006) Automated microELISA system for allergy checker: a prototype and clinical test. Proc Micro Total Anal Syst $1: 858-860$

210. Wang S, Zhao X, Khimji I, Akbas R, Qiu W, Edwards D, Cramer DW, Ye B, Demirci U (2011) Integration of cell phone imaging with microchip ELISA to detect ovarian cancer HE4 biomarker in urine at point-of-care. Lab Chip 11:3411-3418

211. Ozen MO, Sridhar K, Giray Ogut M, Shanmugam A, Avadhani AS, Kobayashi Y, Wu JC, Haddad F, Demirci U (2020) Total 
microfluidic chip for multiplexed diagnostics (ToMMx). Biosens Bioelectron 150:111930

212. Jin JH, Kim JH, Lee SK, Choi SJ, Park CW, Min NK (2018) A fully integrated paper-microfluidic electrochemical device for simultaneous analysis of physiologic blood ions. Sensors 18(1):104

213. Linares EM, Palermo LT, Moreira AB, Taboada Sotomayor MP (2007) A fluorescence spot test for salicylate determination. Anal Lett 40:573-583

214. Silva Lamarca R, de Lima F, Gomes PC (2020) A low cost method for carbamazepine, ciprofloxacin and norfloxacin determination in pharmaceutical formulations based on spot-test and smartphone images. Microchem J 152:104297

215. Usman M, Rasheed H (2019) Bioanalytical techniques used for TDM. In: Babar Z-U-D (ed) Encyclopedia of pharmacy practice and clinical pharmacy, vol 3A. Elsevier, Amsterdam

216. Zhu S, Shimokawa S, Shoyama Y, Tanaka H (2006) A novel analytical ELISA-based methodology for pharmacologically active saikosaponins. Fitoterapia 77:100-108

217. Qian J, He Q, Liu L, Wang M, Wang B, Cui L (2020) Rapid quantification of artemisinin derivatives in antimalarial drugs with dipstick immunoassays. J Pharmaceut Biomed 191:113605

218. Noviana E, Henry ChS (2020) Emerging applications of paperbased analytical devices for drug analysis: a review. Anal Chim Acta 1116:70-90 (and references therein)

219. Antonacci A, ScognamiglioV MV, Caratelli V, Fiore L, Moscone D, Fabiana Arduini F (2020) Paper-based electrochemical devices for the pharmaceutical field: state of the art and perspectives. Bioeng Biotechnol 8:339 (and references therein)

220. Nadolny J (2003) The first century of published scientific analyses of the materials of historical painting and polychromy, circa 1780-1880. Rev Conserv 4:39-51

221. Rees-Jones SG (1990) Early experiments in pigment analysis. Stud Conserv 35:93-101

222. Allen RO (1989). In: Allen RO (ed) Archaeological chemistry IV, advances in chemistry. American Chemical Society, Washington DC, pp 1-17

223. Doménech-Carbó MT, Osete-Cortina L (2016) Another beauty of analytical chemistry: chemical analysis of inorganic pigments of art and archaeological objects. ChemTexts 2:14

224. Laurie AP (1914) The pigments and mediums of the old masters. Macmillan, London

225. Plesters J (1956) Cross-sections and chemical analysis of paint samples. Stud Conserv 2:110-157

226. Mairinger F, Schreiner M (1986) In: van Schoute R, Verougstracte-Marcq H (eds) PACT 13, Xth Anniversary Meeting of PACT Group, Louvain la Neuve, pp 171-183

227. Arnold A (1984) Determination of mineral salts from monuments. Stud Conserv 29:129-138

228. Chamot ET, Mason CW (1957) Handbook of chemical microscopy, vol II, 2nd edn. Wiley, New York

229. Geilmann W (1954) Bilder zur Qualitativen Mikroanalyse Anorganischer Stoffe. Verlag Chemie GmbH, Weinheim

230. Doménech Carbó MT (2018) Análisis químico y examen científico de patrimonio cultural. Sintesis, Madrid (and references therein)

231. Kalsbeek N (2005) Identification of synthetic organic pigments by characteristic colour reactions. Stud Conserv 50:205-229

232. Kostadinovska M, Spirovska ZJ (2015) Implementation of methods for examination of paper-based library materials. Vjesnik bibliotekara Hrvatske 58:119-133

233. BPG spot tests (2021) Book and Paper Group Wiki. American Institute for Conservation (AIC). https://www.conservationwiki.com/wiki/BPG_Spot_Tests. Accessed 12 Jul 2021.
234. Cattaneo C, Gelsthorpe K, Phillips P, Sokol RJ (1992) Reliable identification of human albumin in ancient bone using ELISA and monoclonal antibodies. Am J Phys Anthropol 87:365-372

235. Atrei A, Benetti F, Potenza M, Dei L, Carretti E, Niccolucci V, Marchettini N (2018) Characterization of organic binders in a 13th century painted woodenpanel: comparison of ToF-SIMS and Dot-ELISA results. Int J Mass Spectrom 430:63-68

236. Han Y, Liu Z, Huang X, Wang Y, Zhang Z, Pan J (2020) The application of ELISA to the analysis and research of cementing materials in calligraphy of porcelain relics in Song Dynasty. Microchem J 159:105530

237. Carpenter KJ (2003) A Short History of Nutritional Science: Part 1 (1785-1885). J Nutr 133:638-645

238. Quick test for some adulterants in food, Food Safety and Standards (2012) Authority of India, New Delhi. https://old.fssai. gov.in. Accessed 7 Jun 2021

239. Australian School Science Information Support for Teachers and Technicians (2018) (ASSIST) Laboratory notes: Food tests. https://assist.asta.edu.au. Accessed 21 May 2021.

240. Spot tests for minerals in finished feed (2021) https://www. dairyknowledge.in. Accesssed 7 Jun 2021

241. de Oliveira LMA, Bezerrados Santos V, da Silva EKN, Santos Lopes A, Dantas-Filho HA (2020) An environment-friendly spot test method with digital imaging for the micro-titration of citric fruits. Talanta 206:12021910

242. Dzantiev BB, Byzova NA, Urusov AE, Zherdev AV (2014) Immunochromatographic methods in food analysis. Trends Anal Chem 55:81-93 (and references therein)

243. Han X, Liu Y, Yin J, Yue M, Mu Y (2021) Microfluidic devices for multiplexed detection of foodborne pathogens a Research Centre for Analytical Instrumentation, Institute of Cyber-Systems and Control, State Key Laboratory of Industrial Control Technology. Food Res Int 143:110246

244. Han S, Zhou T, Yin B, He P (2016) A sensitive and semiquantitative method for determination of multidrug residues in animal body fluids using multiplex dipstick immunoassay. Anal Chim Acta 927:64-71

245. Franke WA (2021) Quick assays in mineral identification A guide to experiments for mineral collectors and geoscientists in field work. Freie Universität Berlin. https://www.geo.fu-berlin.de. Accessed 7 Jun 2021

246. Mann VI (1950) A spot test for phosphorus in rocks. J Sediment Res 20:116-117

247. Chan FL (1967) Spot test detection in sulphide minerals. Anal Chim Acta 37:391-393

248. Griffitts WR, Ward FN, Alminas HV (1976) A simple spot test for molybdenum minerals. Econ Geol 71:1595

249. Dutka F, Starchenko V, Osselin F, Magni S, Szymczak P, Ladd AJC (2020) Time-dependent shapes of a dissolving mineral grain: comparisons of simulations with microfluidic experiments. Chem Geol 540:119459

250. Dulio V, van Bavel B, Brorström-Lundén E, Harmsen J, Hollender J, Schlabach M, Slobodnik J, Thomas K, Koschorreck K (2018) Emerging pollutants in the EU: 10 years of NORMAN in support of environmental policies and regulations. Environ Sci Eur 30:5

251. Eddy MD (2008) The language of mineralogy: John Walker, Chemistry and the Edinburgh Medical School, 1750-1800. Rutledge Taylor \& Francis, New York

252. Friederichs K (1958) A definition of ecology and some thoughts about basic concepts. Ecology 39:154-159

253. Pausas JG, Bond WJ (2018) Humboldt and the reinvention of nature. J Ecol 107:1031-1037

254. Sawicki E, Hauser TR (1960) Spot test detection and colorimetric determination of aliphatic aldehydes with 2-hydrazinobenzothiazole. Appl Air Pollut Anal Chem 32:1434-1436 
255. Yue PC, Podzimek J (1980) Potential use of chemical spot test method for submicron aerosol sizing. Ind Eng Chem Prod RD 19:42-46

256. Kamel RM, El-Sakka SS, Bahgat K, Monir RM, Soliman MHA (2021) New turn on fluorimetric sensor for direct detection of ultra-trace ferric ions in industrial wastewater and its application by test strips. J Photoch Photobio A 411:113218

257. Teepoo S, Wongtongdee U, Phapugrangkul P (2020) Development of qualitative and quantitative immunochromatographic strip test assay for rapid and simple detection of leucomalachite green residual in aquatic animals. Food Chem 320:126613

258. Irudayasamy A, Natarajan AR (1961) Spot test microdetermination of DDT and its related compounds in biological materials. Anal Chem 33:630-632

259. Schwemberger JG (2020) Chemical spot test kits for testing for lead-based paint United States Environmental Protection Agency EPA, Science Inventory. https://cfpub.epa.gov/si/si_public_ record_Report.cfm?Lab=OPPT\&dirEntryID=81666. Accessed 6 Aug 2020

260. Soares S, Lima MJA, Rocha FRP (2017) A spot test for iodine value determination in biodiesel based on digital images exploiting a smartphone. Microchem J 133:195-199

261. Soares S, Torres KG, Pimentel E, Martelli PB, Rocha FRP (2019) A novel spot test based on digital images for determination of methanol in biodiesel. Talanta 195:229-235

262. Bogdanovic J, Koets M, Sander I, Wouters I, Meijster T, Heederik D, van Amerongen A, Doekes G (2006) Rapid detection of fungal alpha-amylase in the work environment with a lateral flow immunoassay. J Allergy Clin Immunol 118:1157-1163

263. Liu W, Guo Y, Luo J, Kou J, Zheng H, Li B, Zhang Z (2015) A molecularly imprinted polymer based a lab-on-paper chemiluminescence device for the detection of dichlorvos. Spectrochim Acta A 141:51-57

264. Zhou T, Liu JJ, Xu Y, Wu ZY (2019) Fast and sensitive screening detection of tetracyclines with a paper-based analytical device. Microchem J 145:703-707

265. Arsawiset S, Teepoo S (2020) Ready-to-use, functionalized paper test strip used with a smartphone for the simultaneous on-site detection of free chlorine, hydrogen sulfide and formaldehyde in wastewater. Anal Chim Acta 1118:63-72

266. Pucetaite M, Ohlsson P, Persson P, Hammer E (2021) Shining new light into soil systems: spectroscopy in microfluidic soil chips reveals microbial biogeochemistry. Soil Biol Biochem 153:108078

267. Sicard C, Glen Ch, Aubie B, Wallace D, Jahanshahi-Anbuhi S, Pennings K, Daigger GT, Pelton R, Brennan JD, Filipe CDM
(2015) Tools for water quality monitoring and mapping using paper-based sensors and cell phones. Water Res 70:360-369

268. Mentele MM, Cunningham J, Koehler K, Volckens J, Henry CS (2012) Microfluidic paper-based analytical device for particulate metals. Anal Chem 84:4474-4480

269. Alahmad W, Sahragard A, Varanusupakul P (2021) An overview of the recent developments of microfluidic paper-based analytical devices for the detection of chromium species. Microchem J 170:106699

270. Adegoke AA, Singh G, Stenström TA (2019) Biosensors for monitoring pharmaceutical nanocontaminants and drug resistant bacteria in surface water, subsurface water and wastewater effluent for reuse. In: Grumezescu AM (ed) Nanoparticles in pharmacotherapy. Elsevier, Amsterdam (chapter 16)

271. Arnold E (1933) Chem Listy 27:73 (1933 Brit Chem Abstracts B393)

272. Grasselly G (1952) Electrographical analysis of ore textures. Acta Min Pet VI:47-57 (Univ. Szegediensis)

273. Kronstein M, Ward MM, Roper R (1950) Evaluation of organic coatings by electrographic printing. Ind Eng Chem 42:1568-1572

274. Shaw WE, Moore ET (1947) Pore size in protective films by electrographic printing. Anal Chem 19:777-779

275. Yagoda H (1943) Analytical patterns in the study of mineral and biological materials. N-Holland D 15:135-141

276. Glazunov A, Krivohlavy J (1932) Quantitative Bestimmung des Nickels in Nickelstählen auf elektrographischem Wege. J Physik Chem A161:373-378

277. Alder JF, Baker AE, West TS (1977) The determination of copper in alloys by electrography and atomic absorption spectrometry. Anal Chim Acta 90:267-270 (and references therein)

278. Jensen WB (2017) Remembering qualitative analysis. The 175th Anniversary of Fresenius' Textbook: Part I. Educación Química 28:217-224

279. Soukup RW, Rosner R (2019) Scientific contributions of the first female chemists at the University of Vienna mirrored in publications in Chemical Monthly 1902-1919. Monatsh Chemie 150:961-974

280. Scholz F (2021) Glazunov's electrography-the first electrochemical imaging and the first solid-state electroanalysis. J Solid State Electrochem 25:2705-2715. https://doi.org/10.1007/ s10008-021-04967-1

Publisher's Note Springer Nature remains neutral with regard to jurisdictional claims in published maps and institutional affiliations. 